QC 852

. $C 6$

NSF/NOAA ATM-8419116

no. 394

ARCHIVE

\title{
ENVIRONMENTAL INFLUENCES ON HURRICANE INTENSIFICATION
}

\author{
BY \\ ROBERT T. MERRILL \\ LIBRARIES \\ AUG 291986 \\ COLORADO STATE UNIVERSITY
}

\section{P. I. WILLIAM M. GRAY}

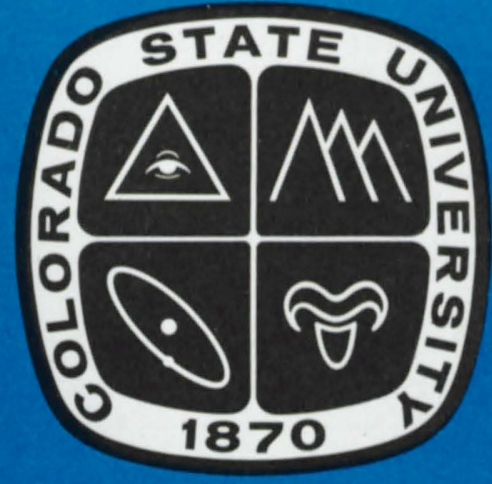

Atmospheric Science

PAPER NO.

394

US ISSN 0067-0340

DEPARTMENT OF ATMOSPHERIC SCIENCE

COLORADO STATE UNIVERSITY

FORT COLLINS, COLORADO 
ENVIRONMENTAL INFLUENCES ON HURRICANE INTENSIFICATION

By

Robert T. Merrill

Department of Atmospheric Science Colorado State University

Fort Collins, Colorado 80523

December, 1985

Atmospheric Science Paper No. 394 


\section{ABSTRACT}

Though qualitatively similar in structure, different hurricanes can attain different peak intensities during their lifetimes. Forecasters and empiricists relate the intensity to the sea surface temperature and the "effectiveness" of the upper tropospheric outflow, but offer no clear explanation of how the latter operates. Numerical modelers usually ignore the surrounding $f l O W$ and emphasize interaction between the convective and vortex scales exclusively. This paper examines more closely the observed upper-tropospheric environmental flow differences between hurricanes which intensify and those which fail to do so, and combines them with previously published empirical and modeling results into a general conceptual model of environmental influences on hurricane intensification.

Upper tropospheric wind observations (from satellite cloud tracking, aircraft reports, and rawinsondes) are composited for 28 hurricanes according to intensity tendency. A rotated coordinate system based on the outflow jet location is used so that the asymmetric flow structure is preserved. Little difference is observed in total outflow on the synoptic scale. However, intensifying hurricanes have a less constricted outflow with evidence of lateral connections with the surrounding flow. The asymmetric flow consists of a wave thought to be associated with barotropic instability of the anticyclonic flow above the hurricane and the juxtaposition of surrounding flow features.

A quasi-equilibrium balance between hurricane convection and the upper tropospheric environment is proposed. The moist-neutral stratification of the vortex core is a balance between the convection which acts to increase stability and the outflow which acts to reduce it. Reduce the outflow layer cooling and the core stabilizes convective buoyancy is reduced, and a new balance with less vigorous convection is established. If vertically sheared, the environmental flow can al so regulate intensity by inducing asymmetric convective structure. Vortex-convection feedbacks are considered to be important mainly in the stages of tropical cyclone development prior to eye formation, which is seen as the first indication that the stabilization process is occurring.

Several observational and numerical tests for this conceptual model are then proposed. 
TABLE OF CONTENTS

Page

1. INTRODUCTION. . . . . . . . . . . . . . . . . . . 1

1.1 The Challenge of Intensification. . . . . . . . . . 1

1.2 Conceptual Thought on the Structure of Hurricanes:

1900-1980................. 4

1.2.1 Large-scale Empiricism: 1900-1957. ...... 4

1.2.2 Turning Inward. ................ 7

1.2.3 CISK, STORMFURY, and Modeling: 1964-1975:.. 9

1.2.4 Composites and P3's: 1976-1980....... 12

1.3 Foundation for the Current Study. .......... 15

1.3.1 Intensity, Strength and Size.......... 15

1.3.2 Dynamical Concepts of Revelance to Cyclone-

1.4 Summary . . . . . . . . . . . . . . . . 20

2. THE COMBINED WIND SET AND ROTATED COORDINATE COMPOSITING

METHOD . . . . . . . . . . . . . . . 22

2.1 Previous Observational Studies of the Outflow Layer . . 22

2.2 Processing of Observations for This Study . . . . . . . 23

2.3 Compositing Methods ................. 25

2.4 The National Hurricane Center Upper-tropospheric

2.5 Individual Case Analysis and Rotation Angle

Determination. . . . . . . . . . . . . . 33

2.6 Discussion of the Composite Method and its Limitations. 49

3. OBSERVATIONS OF THE UPPER TROPOSPHERIC ENVIRONMENT. . . . . . 53

3.1 Outflow Pattern Types for Atlantic Hurricanes . . . . 53

3.2 Sea-surface Temperature Influences on Hurricane

Intensity. .................... 67

3.3 Composites of Intensity and Intensity Change

Normalized According to Sea Surface Temperature. . . 72

3.4 Temporal Changes of Outflow Character During

Intensification. . . . . . . . . . . . . . 80

3.5 Summary of Observational Results. . . . . . . . . 84 
TABLE OF CONTENTS (cont'd)

Page

4. SYMMETRIC AND ASYMMETRIC ASPECTS OF HURRICANE STRUCTURE AND DYNAMICS ................. 88

4.1 Historical Context. ............... 88

4.2 Structure and Dynamics of the High-energy Core. . . . 89

4.3 Thermodynamics Influences on Cyclone Intensity. . . . 93

4.4 Effects of Asymmetric Processes on Symmetric Flow . . . 99

4.5 Review of Wave Properties in Vortices ......... 101

4.6 Characteristics and Observations of Waves in the

Upper Troposphere. ............ 106

5. DISCUSSION. ..................... 114

5.1 Internal Dynamics and Intensity Change. . . . . . . 115

5.2 Environmental Influences on Hurricane Intensity

Change: A Conceptual Model............ 122

5.3 A Research Agenda ................ 132

5.4 Operational Implications............. 136

ACKNOWLEDGEMENTS ...................... 138

REFERENCES ....................... 139

APPENDIX A: W. M. GRAY'S FEDERALLY SUPPORTED RESEARCH PROJECT

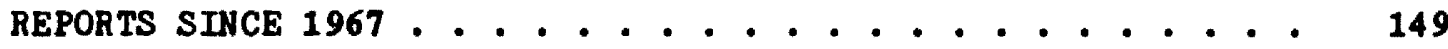




\section{INTRODUCTION}

\footnotetext{
" The barometer had fallen to 26.99 inches. So low a reading had never before been recorded for certain at sea. The dynamics of such a depression were beyond computation." Hughes (1938)
}

\subsection{The Challenge of Intensification}

On Thursday, 29 August 1935 a small tropical disturbance, of "slight intensity," was noted northeast of Turks Island in the eastern Bahamas, shifting slowly westward as is typical of disturbed weather in that latitude. This was before the days of afrcraft reconnaissance, and the Weather Bureau forecaster in Miami could only scan the wireless reports from ships and 1 slands and wonder. Disturbances such as this one sometimes did become hurricanes, but the "big blows" of 1926 and 1928 could be tracked all the way back to the Cape Verde Islands and Africa and were already quite severe upon reaching Puerto Rico, not to mention the Bahamas. This patch of squally weather was only a few days away, and besides, most such rains failed to develop into tropical cyclones at all.

This one, however, did develop into a vortex and gradually it gained power. Sunday, 1 September brought winds of just over hurricane force to Andros Island, in the western Bahamas, and the little hurricane slid into the Florida straits and began the one-day journey to the Florida Keys. Northeast storm and hurricane warnings were posted and the Keys began to get ready. Hurricanes were a part of life for the 
Keys people who had experienced several severe storms since the turn of the century; the less powerful ones were merely a nuisance.

Not everyone was so confident, however. The Great Depression had brought hundreds of unemployed World War veterans to the Keys as part of a Federal project to convert Henry Flagler's bankrupt Overseas Railway Into a motor highway linking Miami and Key West (Parks, 1968). Monday was Labor Day, and some of the workers had been sent to Miami to see a baseball game. At the insistence of the veterans' commander, a special train was made up in Miami and dispatched to pick up the rest of the workers. It never arrived.

Late that Labor Day evening near the north end of Long Key, a barometer owned by Captain Iver 0lson recorded the lowest pressure to this day ever measured in the Western Hemisphere - 26.35 inches (892 $m b)$. Engineers surveying the damage estimated that the winds exceeded $90 \mathrm{~m} \mathrm{~s}^{-1}(175 \mathrm{kt})$. Somehow, the hurricane's central pressure had fallen around $100 \mathrm{mb}$ in just over a day. Over four hundred lives were lost (McDonald, 1935a, 1935b).

September 26, 1955. A Navy "Hurricane Hunter" attempting to locate the center of a rapidly intensifying hurricane named Janet vanishes. Later that day, Janet roars across Swan Island in the western Caribbean Sea with a central pressure of $914 \mathrm{mb}$ and estimated winds of $90 \mathrm{~m} \mathrm{~s}^{-1}$, destroying the weather station (Dunn et al., 1955).

June 27, 1957. June hurricanes are usually weak, but the winds of Audrey unexpectedly increase from 40 to $65 \mathrm{~m} \mathrm{~s}^{-1}$ during the predawn hours, ralsing a $4 \mathrm{~m}$ hurricane surge. Inundation occurs inland as far as $30 \mathrm{~km}$. Five hundred lives are lost in an unprepared Cameron, Louislana (Moore et al., 1957). 
August 3, 1970. The central pressure of Hurricane Celia fal is 43 $\mathrm{mb}$ in the 15 hours prior to landfall. Strong west winds devastate the Corpus Christi, Texas area, but the deadly storm surge fortunately pours inland several miles to the north, over a sparsely populated stretch of coast (Simpson and Pelissier, 1971).

August, 1980. A hurricane named Allen holds a convincing demonstration of sudden intensity change with 24 hour drops of over 40 mb on three separate occasions, and mercifully fills a similar amount in the 12 hours prior to landfall (Lawrence and Pelissier, 1981).

What is happening? What causes some hurricanes to quickly attain record-breaking destructiveness, while others can barely hold their own? What causes these periods of rapid intensification? Can they be predicted? Are they the result of some deeply hidden rearrangement of the hurricane's inner workings, or do they stem fram some passing influence in the hurricane's surroundings? Scientific questions of scale interaction between the cumulus, meso-, and synoptic scales are Involved, as are the physical processes of afr-sea interaction. Operationaliy, intensity change prediction is an area which has recelved little attention, but the dangers of a rapid intensification prior to landfall are beginning to be realized (Carter, 1985). Prepare for what a minimal hurricane could become in 24 hours and you will have gross overwarning most of the time. Assume that a minimal hurricane will stay that way and in 24 hours you could concelvably have the disaster of the century.

This paper attempts to present the state of knowledge about hurricane intensity change as of 1985 , particularly the role played by the hurricane's upper-tropospheric surroundings. Our knowledge of 
hurricane structure and dynamics has progressed over the years making new interpretations of older research possible and desirable. The remainder of this first chapter summarizes this valuable work of the last half century and indicates where re-interpretations can be profitably made. Chapter 2 describes a new combined upper tropospheric wind set which is ideal for studying the outflow layer, and a new compositing technique used to process it. Chapters 3 and 4 contain the original research on environment-hurricane interactions which are the thrust of this paper. Chapter 5 synthesizes the past and current research into a conceptual model of environmental effects on hurricane Intensity, and outlines areas where additional work is essential if this challenging problem is to be fully grasped. The operational Implications of this work are also briefly discussed. 1.2 Conceptual Thought on the Structure of Hurricanes: 1900-1980

\subsubsection{Large-scale Empiric1sm: 1900-1957}

Al though the broad, rotary character of hurricanes had been known for almost a century beforehand (Redfleld, 1831), the first salentific study was a book entitled simply Iropical Cyclones, published in 1926 . The author, Isaac Cline, was the forecaster at Galveston during the 1900 hurricane (which claimed 6000 lives), and analyzed this and several other hurricanes by compositing the hourly surface observations from coastal stations with respect to the moving vortex. The cyclonic, inflowing circulations, highest winds surrounding a relatively calm and cloud-free center or "eye," and the copious rainfall ahead and to the right of the vortex were all noted. Little could be sald about the vertical depth of what was considered to be essentially a low-level phenomenon, as the only upper winds were those obtalned by tracking 
clouds at various heights. These were few in number and understandably confusing.

Controversy regarding the depth of the hurricane vortex persisted into the 1930 's, until Haurwitz (1935) demonstrated from hydrostatic considerations that a surface pressure reduction of the magnitude found In a hurricane must involve warm air through a great depth. The beginnings of instrumented balloon ascents at about the same time provided supporting observational evidence. Haurwitz (1935) also Inferred the subsident character of the eye and the broadening with height of the warm core.

Interest in hurricanes and their Pacific counterparts the typhoons was accelerated by the Second World War in which the U.S. and Japan were maintaining a sizable naval presence in the tropical Pacific Ocean. Extensive damage to the U.S. Pacific fleet in 1944 and 1945 led to the establishment of specialized typhoon forecast centers and systematic tracking of hurricanes and typhoons by alraraft. Empirical rules for locating developed tropical cyclones and predicting their motion were put forward, and by the mid $1950^{\prime} \mathrm{s}$, much of the qualitative knowledge of the large-scale character of hurricanes was already in place. Riehl (1954) made the following statements which are still regarded as valid:

1) Hurricanes are synoptic-scale cyclones of the tropics and subtropics which form and exist in a generally barotropic atmosphere and (In contrast to extratropical cyclones), are warm-cored throughout the depth of the atmosphere.

2) The energy source for hurricanes is the condensation of water in convective clouds. 
3) Air flows into the hurricane in the low levels and out of the hurricane in the upper levels.

4) Hurricanes move within the same general velocity as the air in which they are embedded, particularly in the mid levels.

5) Hurricanes form from pre-existing synoptic disturbances in the tropics.

Though they can now be stated more precisely, these qualitative statements have been confirmed with no major modifications over the last 30 years. The realiy difficult problem in Riehl's time was to explain how a hurricane started. Herbert Riehl used to repeatedly ask his students, "What makes a hurricane?" and the problem of intensity change was seen as related to the more difficult formation question. Willett (1955) saw the hurricane as an essentially convective system, and concluded that cold air aloft, in addition to warm sea-surface temperatures, would enhance convection and thus aid the formation process. As evidence of this he cited the unsettled, showery weather which made up the "pre-existing disturbances" from which hurricanes formed. The "triggering mechanisw", if any, was seen as the advection of cold air aloft. This "convective theory" dominated through the 1930 's but came under Increasing crit1cism later (Dunn, 1951). Others. represented by Riehl (1951), Sawyer (1947), and Dunn (1951) sought to Invoke a dynamic explanation Involving the superposition of divergence aloft over an existing disturbance, triggering the inftial pressure fall and vortex development. It was concerning this "outflow layer" that the main controversy of the 1950's arose, leading Dunn (1951) to state:

- The problem of how evacuation of air from tropical cyclones takes place is the most controversial portion of the theory of tropical cyclone structure". 
The ultimate intensity reached was considered to be thermodynamically controlled: Willett (1955) related it to the helght of the tropopause and thus the depth of the convectively unstable environment, while Dunn (1951) invoked differences in the moisture and stability of the inflowing air during the period of rapid deepening which followed the formation of the vortex.

These explanations of hurricane formation and intensification were almost entirely qualitative and based upon descriptive studies rather than theoretical first principles. By modern standards, data were sparse to non-existent, particularly near the hurricane center and above the surface. It is a testimony to foresight of these scientists that the topics which they identified as controversial are still being discussed today.

\subsubsection{Turning Inward: 1958-1963}

Events in the science were already afoot that would radically al ter our view of the hurricane away from the broad-scale, empirical image held by the forecaster with his synoptic maps. The pioneering work of Charney (1947) and others with midlatitude dynamics had led to the development of the quasigeostrophic model and the use of linear perturbation stability analysis - cornerstones of atmospheric dynamics. Proper scale analysis could transform the complex, nonlinear primitive equations into a reasonable description of atmospheric motions, and perturbation analysis could identify conditions under which disturbances could amplify. Four severe hurricanes (Carol and Hazel in 1954, Connie and Diane in 1955) struck the east coast of the United States, and the hurricane became the prime target of meteorological research. 
A special National Hurricane Research Project (NHRP) was established and plans made to fly instrumented aircraft into hurricanes to collect detafled measurements. New forecasting techniques would be sought, and the developing technology of electronic computing would be brought to bear.

This new approach, almost out of its very nature, brought with it a turning inward. The limited range of research alroraft and the heretofore unknown core structure of the hurricane meant that the flights would be conducted so as to get the most data within $100 \mathrm{~km}$ of the center. The approach of perturbation analysis and the conceptual view it tends to engender involve perturbations on the simplest possible basic state which, in the case of the hurricane, is a uniform, barotropic atmosphere at rest.

The collection of data was rapid and spectacular. As with any new field program, old models were discarded and new ones introduced as the data were processed. Not surprisingly, the hurricane became smaller. Conceptually, the new observations were interpreted in light of the old empirical concepts at first, but as the flight data began to make quantitative studies of inner-core processes possible, the interest in qualitative interpretations of the flight data involving scales larger than the inner core began to decline. The question of hurricane formation led to two schools of thought. The first, following the tradition of empiricism, saw the formation of a hurricane as being regulated, or at least triggered, by changes in the large scale flow. Barotropic, baroclinic, and inertial instability were all proposed, but the hypothesized mechanisms were sufficiently complex that a closed, mathematically satisfying perturbation analysis could not be performed. 
A second school of thought developed around the possibility of an instability associated with the convective processes and their interaction with the developing vortex. A puzzling question at the time was the question of latent heat release and 1 ts effects on the larger scales; do hurricanes have more latent heat release than other tropical disturbances, or do they better utilize a similar quantity? Linkage of either the amount of latent heat release or its utilization to the stage of development of the tropical cyclone would constitute the sought-after instability.

This concept that the convection and vortex, though of different scales, could cooperate was termed CISK (Conditional Instability of the Second kind). CISK was first described by Doyama (1964), and a linear stability argument was put forward by Charney and Eliassen (1964). Although the assumptions involved were recognized at the time, the CISK hypothesis was so crisp and clean that it quickly came to dominate conceptual thought on hurricanes to the degree that it became the basis for much of the research of the next decade.

\subsubsection{CISK, STORMFURY, and Modeling: 1964-1975}

With the concept of Iinear CISK described, the path to understanding the hurricane seemed to lay in increased research flying in the core and simulation of the nonlinear aspects of CISK using numerical models. As CISK was concerned with low level moisture convergence, friction, and wind structure, the upper-tropospheric factors of interest to empiricists recelved less and less attention, and the last upper-tropospheric research flight was made in 1966. Additional emphasis was placed upon convection-cyclone interactions by Project STORMFURY, the plan to reduce the maximum winds in hurricanes. 
The initial STORMFURY hypothesis (Simpson and Malkus, 1964; Gentry, 1969) called for the reduction of horizontal temperature gradients and hence maximum winds by seeding the region just outside the hurricane eye with sllver lodide, causing supercooled water to freeze and liberate heat of fusion. The hypothesis changed with time but the underlying assumption was the same; internal processes were dominant and held the key to intensity change. STORMFURY involved many assumptions and the logical place to test them was a numerical model, the integration of which was in itself a scientific challenge of the first order. The period 1964-1975 began with the ideas far ahead of the experiments, and 1t was only towards the end of that period that the technology of airoraft instrumentation and data processing and methods of numerical modeling had progressed to the point where they began to deliver a return on the massive investment of ef fort and resources they required. The creative explosion begun in the $1950^{\prime} \mathrm{s}$ largely subsided, and the conceptual view of the hurricane, circa 1975, was a nonlinear, complex version of the internal 1nstability CISK model of 1964, whereby a weak vortex developed into an intense hurricane in a passive envirorment. Research on environmental factors (with the exception of those involving motion and therefore track prediction) stagnated, and papers written on the large-scale aspects of hurricanes remained largely qualitative and outside the mainstream of hurricane research.

The ability of researchers to simulate a hurricane mathematically grew by leaps and bounds, paced both by rapid increases in computer power and by advances in numerical methods and representations of the relevant physics, particularly molst convection. Anthes (1982) provides an excellent summary of the progress of hurricane modeling. Hurricane 
models have simulated the development process, including the formation of the eye, and have indicated a sensitivity to the sea surface temperature and initial distribution of moisture. The vertical distribution of latent heat release has al so been found to be critical to the development of the vortex. With a few noteable exceptions (Mathur, 1974; Kurihara and Tuleya, 1981; and Challa and Pfeffer, 1980), numerical models have treated the hurricane as a perturbation on a benign, often stationary basic state. Increases in computing power have gone into higher resolution of the core structure and physics, without similar emphasis being placed on a more realistic treatment of the surrounding flow. The lack of high-quality data on the larger scales, the difficulty of inftializing with real data in the tropics, and the strength of the "passive environment" conceptual view held by the modeling community have all contributed to the neglect of the cycloneenvironment interactions which are the topic of this paper. Nevertheless, much of our current understanding of the hurricane has been made possible by our recently developed ability to solve the equations of motion numerically.

Large-scale influences on hurricane behavior were not forgotten altogether. Efforts at improved track predictions by objective means (statistical and numerical) proceeded at a furious pace from 1964-1975, but much of this work was dedicated to improving the technology by which the "steering flow" hypothesis of the mid 1950's was applied in practice. Studies of the large-scale influences on the development and structural changes of hurricanes were limited primarily to qualitative case studies and empirical rules for forecast centers. Speculation concerning relationships between the environment and hurricane of ten 
accompanied these "rules of thumb", but they could not be proven to the satisfaction of the scientific community. Forecasters went on using them all the same. Examples are found in Simpson (1971) and Hebert (1978).

\subsubsection{Composites and P3's: 1976-1980}

Towards the middle 1970's the impetus of model development began to slow, and a greater variety of experiments using existing technology began to appear. The winding down of Project Stormfury and the acquisition by NOAA of three aircraft equipped with state-of-the-art navigation, sensing and recording systems heralded a renalssance of research flying. The development of operational geosynchronous satellites equipped with IR sensors allowed true continuous mont toring of tropical cyclones, and also for the multiplication of lower and upper-troposphere wind measurements by cloud tracking. The maturing of the experimental technologies of the past 15 years was setting the stage for another period of advancement in conceptual thought in the research community.

The observational research community was also beginning to put the new technology of computer processing to work in new ways. Research using weather observations had always consisted of a careful case study of a particular event, often using data specifically collected for research purposes. Processing was slow and the data were always sparse enough over the tropical oceans that quantitative hurricane work was difficult. Even if successful, the analysis was strictly valid only for a single case. But with computer archiving, retrieval, and processing, it became possible to make a "composite" by averaging many years of rawinsonde observations originally collected for operational use. 
Soundings were averaged together according to their location on a grid centered on the phenomenon under study. With a sufficient number of occurrences of the phenomenon and a few soundings per occurrence, the mean soundings at various locations on the grid grew sufficlently robust that a quantitative picture of the average phenomenon could be had. The method was applied first to tropical cloud clusters (Williams, 1970; Williams and Gray, 1973; Reed and Recker, 1971; Reed and Johnson, 1974; Reed et all., 1977) and then to tropical cyclones (Frank, 1977a; McBride, 1980). The method of "rawinsonde compositing" was slow to gain acceptance, but has in the past decade yielded much information about the large scale aspects of tropical cyclone structure, formation, and movement that could not have been obtained in any other way.

The following results were obtained from rawinsonde composite analysis under Prof. W. M. Gray of Colorado State University during the period 1975-1980:

1) Tropical cyclones were associated with a disruption of the large scale flow to a greater spatial extent than previously thought. Frank (1977a) presented a composite analysis of a typhoon in which low level cyclontc flow extended outward to over 15 degrees latitude (1600 km) radius.

2) Tropical cyclone inflow at radil beyond a few hundred $\mathrm{km}$ was very deep rather than being confined to the boundary layer.

3) The tropical disturbances which ultimately developed into tropical cyclones were on an average located in a different envirormental flow than those which did not. Low level cyclonic and upper-level anticyclonic flow were stronger at $6^{\circ}$ latitude radius around the pre-cyclone disturbance. Since tropical cyclogenesis was widely 
held to be a mesoscale process, the differences could not be attributed to the development process itself.

4) Cyclogenesis was associated with the development of a smaller, organized vortex within the "envelope" described in (3).

With each new observation from application of the compositing approach there arose a qualitative explanation and physical hypothesis attempting to relate the observed large-scale difference to changes in the structure and/or energetics of the hurricane. Some of these hypotheses have been examined and rejected in the light of later results, but advances in our understanding of hurricane structure and dynamics on the smaller scales are making it possible to re-interpret some of the earlier results of rawinsonde compositing.

This has been particulariy true of the topic of intensity change. Nunez (1981) compared intensifying and non-intensifying tropical

cyclones of both Atlantic and Pacific Oceans using composite methods and found that net vertical motion within $4^{\circ}$ latitude of the center was no different. This finding supported the earlier results of Arnold (1977) that the area occupied by penetrative convection actually diminished as a system went from disturbance to troplcal storm. It was therefore concluded that stimulation of the mass circulation by enhanced outflow (Sadler, 1978) was not an important mechanism. We have since come to understand that the concentration of vertical motion and convection near the center is what is important. This concentration was indeed observed by Arnold (1977), but its full significance is only now being realized. 


\subsection{Foundation for the Current Study}

\subsubsection{Intensity, Strength, and Size}

Some of the disappointments we have encountered in our efforts to understand the hurricane have resulted from our incomplete awareness of the natural variability of tropical cyclone structure. of paramount importance was our tendency to forget that hurricane circulations could vary in horizontal extent as well as amplitude. We tend to describe a hurricane stature in terms of its maximum low-level wind or minimum sea level pressure alone, and work outward from there. In some instances (often in modeling work) the radius of maximum winds was also noted, but in the vast majority of cases the remainder of the circulation went undescribed.

Arakawa (1950) and Brand (1972) described some aspects of tropical cyclone size for the Pacific basin and Merrill (1982, 1984b) presented size climatologies for Atlantic and Pacific. Exceptionally large and small cyclones were found to occur in preferred regions and seasons, and Brand (1970) and Merrill (1982, 1984b) explained these differences in terms of environmental vorticity differences and low level angular momentum imports. Merrill (1982, 1984b) compared angular momentum contents for cyclones for varying sizes and intensities and found, quite reasonably, that size overwhelmingly determined angular monentum content. This conclusion explained the difficulty that several investigators (Pfeffer, 1958; Hebert and Jarvinen, 1977) had in relating angular momentum fluxes at synoptic radil to intensity changes. The implicit assumption that more angular momentum meant a higher maximum wind is flawed; often cyclones which are growing rapidly (or 
equivalently gaining angular momentum) are actually diminishing in intensity.

The use of multi-year USAF operational reconnalssance data by Weatherford and Gray (1984), Gray (1984) and Weatherford (1985) has led to the description of tropical cyclone variability in strength - a scale In between intensity and size. Figure 1.1 shows these three scales of variability. Weatherford (1985) has shown that intensity and strength changes over $12 \mathrm{~h}$ intervals are independent. For a given strength, eye character and intensity are related, with more intense typhoons tending to have small eyes. Some internal physical processes or environmental Influences may affect strength directly, and others act to change the eye character and intensity, and careful distinctions must be made. Any explanation for intensity change must involve a physical process which 1s: 1) varlable on time scales (corresponding to periods of intensity change 24-48h duration, and 2) exerting a greater influence on the core than anywhere else. With this in mind many older concepts can be reevaluated with the intensity change problem in mind. Two such concepts, balanced axisymmetric vortex dynamics and angular momentum imports, will be discussed in the next subsection.

1.3.2 Dynamical Concepts of Relevance to Cyclone-environment Interactions The terms "tropical cyclone" and "envirorment" are admittedly vague. While it is not the purpose of this paper to propose a strict definition (nor is such a definition desirable given the continuous nature of fiuid motions) it would be helpful to describe dy namically what types of processes are associated with the "cyclone" and "envirorment". The angular momentum budget equation in cylindrical coordinates provides a convenient means for dolng this, and also allows 


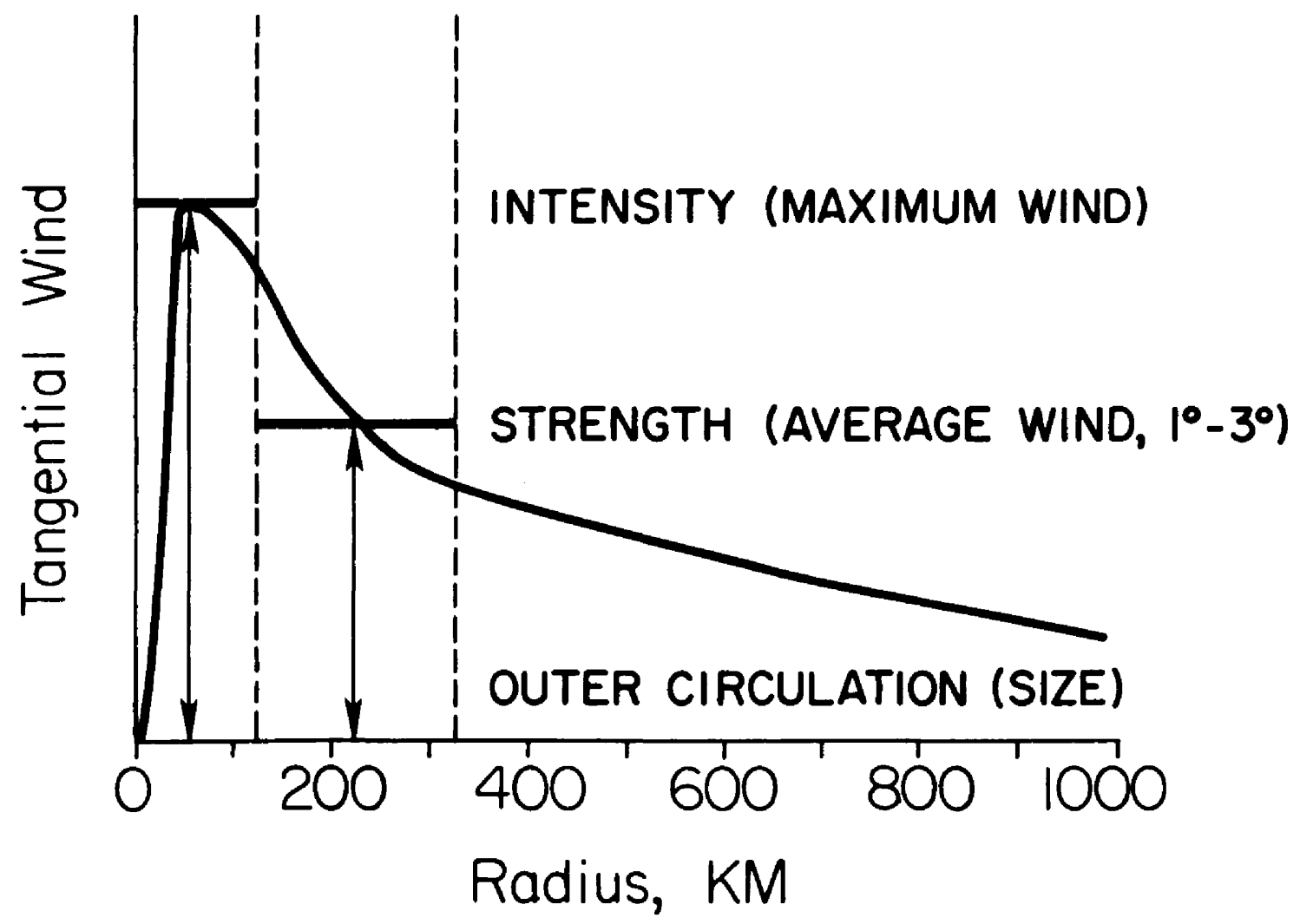

Fig. 1.1. Schematic of variability of the low level tangential wind field as indicated by intensity, strength, and outer circulation.

for discussion of aspects of angular momentum transports which are relevant to intensity change.

Where does the cyclone end and the environment begin? One measure, that of the radius of the outer closed 1sobar, was used by Brand (1972) and Merrill $(1982,1984 b)$ as a measure of troplcal cyclone size. The outer closed isobar will be the one at which the pressure gradient associated with the cyclone just slightly overpowers the superimposed the "enviromental" gradient. Another measure is the distance from the cyclone center to the "neutral point" in the wind field, or the radius at which the mean tangential wind in cylindrical coordinates is zero. Another possible measure is the extent of the cloud shield 
(Arnold, 1977). Radius of Quter Closed Isobar (ROCI) and cloud shteld radius glve similar values $\left(4.5^{\circ}\right.$ latitude and $5.5^{\circ}$ latitude respectively for typhoons) while rawinsonde composites show that, in the mean, the wind-based criteria give radil of more than $15^{\circ}$ latitude. These types of measurements are physically reasonable for a vortex superimposed on an environmental flow which is nearly uniform in the horizontal, but when the enviroment itself contains horizontal shear and/or curvature, ambiguities result with these "kinematic" definitions.

An alternative is to examine the hurricane dynamically. Angular momentum is a convenient quantity to examine since it is conserved in the absence of torques. Formulating the tangential equation of motion in pressure coordinates and integrating in azimuth eliminates the pressure torques so that the only significant torque is that imposed by surface stresses. In a hurricane, these always act to spin down the cyclonic flow, so that, in order to persist, the hurricane must import Increasing quantities of angular momentum with radius. Pfeffer (1958) and Holland (1983) have used observed winds to estimate the manner in which these imports which act to offset surface stresses occur. At Inner radil, the import is primarily by an axisymmetric radial circulation with inflow at low levels (and positive tangential winds) and outflow at high levels (negative tangential winds). At larger radi1, the import is dominated by horizontal asymmetries, mainly in the upper troposphere; inflow is associated with weakly positive tangential winds, and outflow with strongly negative tangential winds. The relative magnitudes of "mean" and "eddy" transports provide a convenient distinction between "cyclone" and "enviroment". The 
environment is dominated by horizontal eddies which are necessary (from an angular momentum standpoint) for the maintenance of the cyclone. The existence of these eddy imports poses some interesting challenges. Axisymmetric models cannot represent them at all, and must either use an extremely large domain such that the hurricane is a closed system for momentum purposes, or employ a boundary condition which implicitly imports angular momentum. Three-dimensional models can and do develop asymmetries, but unless a "realistic" environment is used they are merely the result of inertial instabilities in the outflow layer (Anthes, 1972) and do not increase outward to large radil as do those observed in real hurricanes. Pfeffer and Challa (1981) and Challa and Pfeffer (1980) have attempted to Include the eddy effects in an axisymmetric model by specifying them as part of the forcing terms (along with diabatic heating and frictional stress). Depending on the vertical structure of the imposed eddies, a variety of vortex behavior can be obtained, including various combinations of intensity and size changes. Their resul ts are discussed in Chapter 4.

Studies of environment-cyclone interactions by Pfeffer and Challa (1981) and of eye dynamics by Shapiro and Willoughby (1982) have both employed the balanced vortex (Eliassen, 1951), indicating its power and usefulness. The response of balanced vortices to stresses (momentum or heat sources) depends on the ratio of inertial to static stability; the higher the ratio, the more horizontally constrained is the response. Holland and Merrill (1984) discuss this phenomenon extensively in connection with intensity change. As was discussed in the previous subsection, Intensity appears to be associated with changes in the hurricane core only so any environmental influence must therefore occur 
at a level where the inertial stability at all radil is sufficiently low to allow lateral coupling. This condition is found in the upper troposphere, which at any given radius inside $1000 \mathrm{~km}$, is less resistent to horizontal motions than the lower troposphere. The upper troposphere is exactly where empiricists (Ramage, 1959, 1974; Riehl, 1948; Sadler, 1978; and Simpson, 1971) have noted the apparent relationship between outflow and intensity change. It is therefore in the upper troposphere that this study of environmental influences on hurricane intensity change will concentrate.

\subsection{Summary}

This paper alms to describe the influences which the enviromental flow (region beyond $500-1000 \mathrm{~km}$ radius where horlzontal asymmetries dominate) exerts on the structure, particularly the maximum winds, of the hurricane.

It is implicitly assumed that an influence does exist, and that the conclusions to that effect of qualitative large-scale studies published since the late 1940's are correct. The physical explanations of previous empirical studies will be re-evaluated in terms of the progress made in our understanding of the core structure, and an internally consistent hypothesis of cyclone-enviroment interactions proposed.

The synoptic scale structure of the outflow layer will be studied using a combined wind set containing satellite cloud track winds, aircraft reports, and rawins. This data set and the rotated coordinate system used to process it are discussed in Chapter 2. A discussion of Atlantic basin pattern types, and the large scale outflow differences between intensifying and non-intensifying hurricanes under various conditions is contained in Chapter 3 . 
Chapter 4 describes the nature of wave processes in vortices which are the manifestation of hurricane-environment interactions. The qualitative link between the outflow characteristics discussed in Chapter 3 and the vortex response 1s then made in Chapter 5. Potential operational development of the resul ts of this work and suggestions for further research are also outlined. 
2. THE COMBINED WIND SET AND ROTATED COORDINATE COMPOSITING METHOD

2.1 Previous Observational Studies of the Outflow Layer

The first and foremost objective of this research is to document systematically the synoptic scale structure of the tropical cyclone outflow layer - a necessary condition for physical understanding but a seemingly neglected subject. The outflow layer studies by Ramage (1959, 1974), Riehl (1948), and Sadler (1978) are limited to a few cases, and are largely qualitative. Studies of inertial instability by Alaka (1961, 1963) are quantitative but the results are again based on only a few cases. Another observational study of outflow layer kinematics by Black and Anthes (1971) relied heavily upon the winds derived from cloud motions on geostationary satellite imagery and thus contained more reliable and consistent analyses than the work clted above; its main drawbacks were the relatively small area of analysis and number of cases. This may be a reflection of the consensus of that day that the outflow layer was passively driven by the convection and therefore decayed with increasing radius. Black and Anthes (1971) nonetheless convincingly demonstrated the existence of asymmetries in the outflow layer of hurricanes, even at inner radil.

Most of the observational studies on large-scale hurricane structure have been conducted by the research group of Professor W. M. Gray of Colorado State University, using rawinsonde compositing methods. As was stated in Chapter 1, these methods revealed many previously unknown features of the large-scale structure of hurricanes. However, 
one of the drawbacks of composite analysis is that important features which vary in location from case to case tend to be smoothed so that composite hurricanes appear more nearly axisymmetric than do the individual cases from which they are produced. This loss of the asymmetric structure can be partially overcome if the relevance of it can be mathematically represented in flux form and the flux computed for each individual sounding. The effects of the asymmetries can then be seen as a residual in budget calculations (Frank, 1977b). However, if the relevance of the asymmetries is not known, or if calculation of their mathematical formulation requires the knowledge of horizontal or temporal gradients, the asymmetries are eas1ly overlooked.

Analysis of the outflow layer is thus a complex matter. Data are still usually insufficient to resolve quantitatively the flow in an Individual case (although the avallability of satellite winds has made the upper-troposphere the least data-sparse level in the tropics). Objective analysis methods are also more difficult to implement than in the midlatitudes because of the lack of coupling between mass and momentum fields, and the importance of the divergent component in convective areas. Composite analysis smooths the asymmetry in outflow structure which is thought to be important.

\subsection{Processing of Observations for This Study}

It was apparent from the start that a proper analysis of the outflow layer would have to make use of satellite-derived winds, and that some means of compositing the data without smoothing away the outflow asymmetries would have to be found. Nost studies using satellite winds have relied on the "custcon" extraction of winds using an interactive image processing system. The result is a data set of 
higher quality but, because of the expense and effort involved, of a limited number of cases. The alternative of using an operational set was chosen when it was found that raw data as well as analyzed fields had been archived at the National Hurricane Center (NHC). The winds are of lower quality and contain errors which had to be located and corrected, but the operational set has the advantages of a longer period of record, and the inclusion of rawinsonde winds and winds from commercial aircraft in addition to the satellite cloud track winds. The characteristics of this data set will be described in the next section. Potential problems which can arise from the use of several observing platforms with different characteristics will be discussed in section 2.4 .

New compositing software was written to allow for the large size of the upper-tropospheric features (ridges and troughs of the same scale as midlatitude longwaves; Riehl, 1948) and the asymmetric and variable nature of the tropical cyclone outflow channels. Compositing of upper tropospheric winds is done on two different grids: a 9 X 13 Cartesian grid covering a $4500 \times 6500 \mathrm{~km}$ domain (500 km grid spacing), and a 15degree radius $(\sim 1700 \mathrm{~km})$ polar-coordinate grid, very similar to that used for numerous rawinsonde compositing applications (Frank, 1977a). Both grids can be rotated, using an angle specified with each tropical cyclone position. A complete description of the compositing technique is given in the following section.

In the compositing process, the grids are positioned on the Earth's surface relative to the location of the tropical cyclone's center and oriented at a specified rotation angle (1f the grid rotation option is in use). Tropical cyclone (maximum winds of $17 \mathrm{~m} \mathrm{~s}^{-1}$ or more) positions 
and intensities at $6 \mathrm{~h}$ intervals were obtained from the NHC HURDAT tape (Jarvinen and Case, 1978), and interpolated to $1 \mathrm{~h}$ intervals using the Akima (1970) bivariate interpolation. Motions are computed using centered differencing on the hourly positions. Positions, motions, and wind speeds at 00 and $12 \mathrm{GMI}$ daily are retained for use in the composite program. Intensity changes for each position are represented by the change in best track maximum wind over a 24-h interval centered on the position time.

Initial runs to test for wind data bias caused by the different observing systems (section 2.4) are made using all named tropical cyclone positions in the Gulf of Mexico region (poleward of $18 \mathrm{~N}$ and west of $80 \mathrm{~W}$ ) regardless of intensity, and without the grid rotation option. Subsequent tests of the effect of grid rotation and production runs of specific pattern types and intensity tendencies required further enhancements to the tropical cyclone position file; the removal of all tropical storms (17 $\mathrm{m} \mathrm{s}^{-1} \leq$ maximum wind $\leq 32 \mathrm{~m} \mathrm{~s}^{-1}$ ) and assignment of a rotation angle and an outflow pattern type (both based on streamline analysis of the winds for each case). Th1s process is described in section 2.5. Various subsets of the hurricane position file were then used to generate the composites described in sections $3.1,3.3$ and 3.4 .

\subsection{Compositing Methods}

Composite analysis of weather systems has come into widespread use, particularly for the treatment of weather systems in data sparse areas such as the tropics. (Frank, 1977a; Reed and Recker, 1971; Williams and Gray, 1973). The technique is potentially quite powerful because it allows a generalized quantitative treatment of weather systems without resorting to the assumptions required by objective analysis with limited 
observations and the conclusions which are drawn are more general than those from individual case studies. Composite methods are also a challenge to properly apply and interpret and may miss the relevant features of a weather system al together if not done carefully.

A good composite of the hurricane outflow layer which properly depicts the asymmetric structure has never before been prepared. The problem lies in the navigation of the compositing grid with respect to the the principal outflow jet in addition to the location of the hurricane. Determining the outflow jet location requires an analysis of the flow field for each case. Such a process is difficult using rawinsonde data alone, but possible, although time-consuming, with the combined (satellite wind, alrcraft wind, rawinsonde) set. Addition of this extra step of case-by-case analysis is felt to be essential if a composite representation of the outflow asymmetries is to be had.

Design of the composite grid was based on the scale of the outflow structures to be studied and the desire for adequate data density within each grid box. Qualitative discussions of the upper-tropospheric flow around hurricanes (Riehl, 1948; Ramage, 1959; Sadler, 1978) indicate that the outflow occurs in association with synoptic scale features such as troughs in the westerlies or upper-tropospheric cold lows. The author has repeatedly observed a characteristic spacing of $15^{\circ}-20^{\circ}$ longitude between tropical disturbances, apparently associated with the pattern of upper-tropospheric troughs and ridges, so a domain coverage of slightly more than one such "wavelength" on all sides of a hurricane in the center of the grid was chosen. The grid spacing of 500 $\mathrm{km}$ was selected to be fine enough to resolve synoptic scale features, while coarse enough to provide an average of at least 1-2 observations 
per grid box per case to allow subjective analysis of the outflow jet location in Individual cases.

The grid is shown in Fig. 2.1. It is a $9 \times 13$ array of points 500 $k m$ apart, centered on the location of the hurricane. It is projected onto the Earth's surface using an oblique cylindrical projection (Shapiro and Neumann, 1984) and can be rotated so that the middle column occupies any desired orientation. This grid (referred to as the "Cartesian" grid) is used for both individual case analysis and compositing. To allow direct comparisons with the rawinsonde compositing results already available at CSU, a polar coordinate grid (Fig. 2.2) of $15^{\circ}$ latitude radius was also used for compositing data from the upper tropospheric wind set. The polar grid consists of radial belts of $1^{\circ}$ latitude width out to $7^{\circ}$ latitude radius and $2^{\circ}$ latitude width thereafter, with each belt further subdivided into octants. Like the Cartesian grid, the orientation of Octant 1 of the Polar grid can be changed at will for outflow-jet resolution. This polar grid provides a much finer resolution of the interface region between the subsynoptic scale hurricane vortex (upper-tropospheric radius of $500 \mathrm{~km}$ or less) and the synoptic-scale features of the outflow at radil of $2000-4000 \mathrm{~km}$ as represented on the Cartesian grid.

2.4 The National Hurricane Center Upper-tropospheric Wind Set Beginning in 1974 (when operational geostationary satelitte imagery became available), the NHC autconated their upper troposphere analysis and added high-level cloud motions and winds reported by commercial aircraft to the data base of $200 \mathrm{mb}$ rawinsonde observations. Winds are then objectively analyzed twice dally using a Cressman scheme on a 2800 point grid ranging from $10^{\circ} \mathrm{S}$ to $45^{\circ} \mathrm{N}$, and from $0^{\circ} \mathrm{W}$ to $125^{\circ} \mathrm{W}$ and the raw 


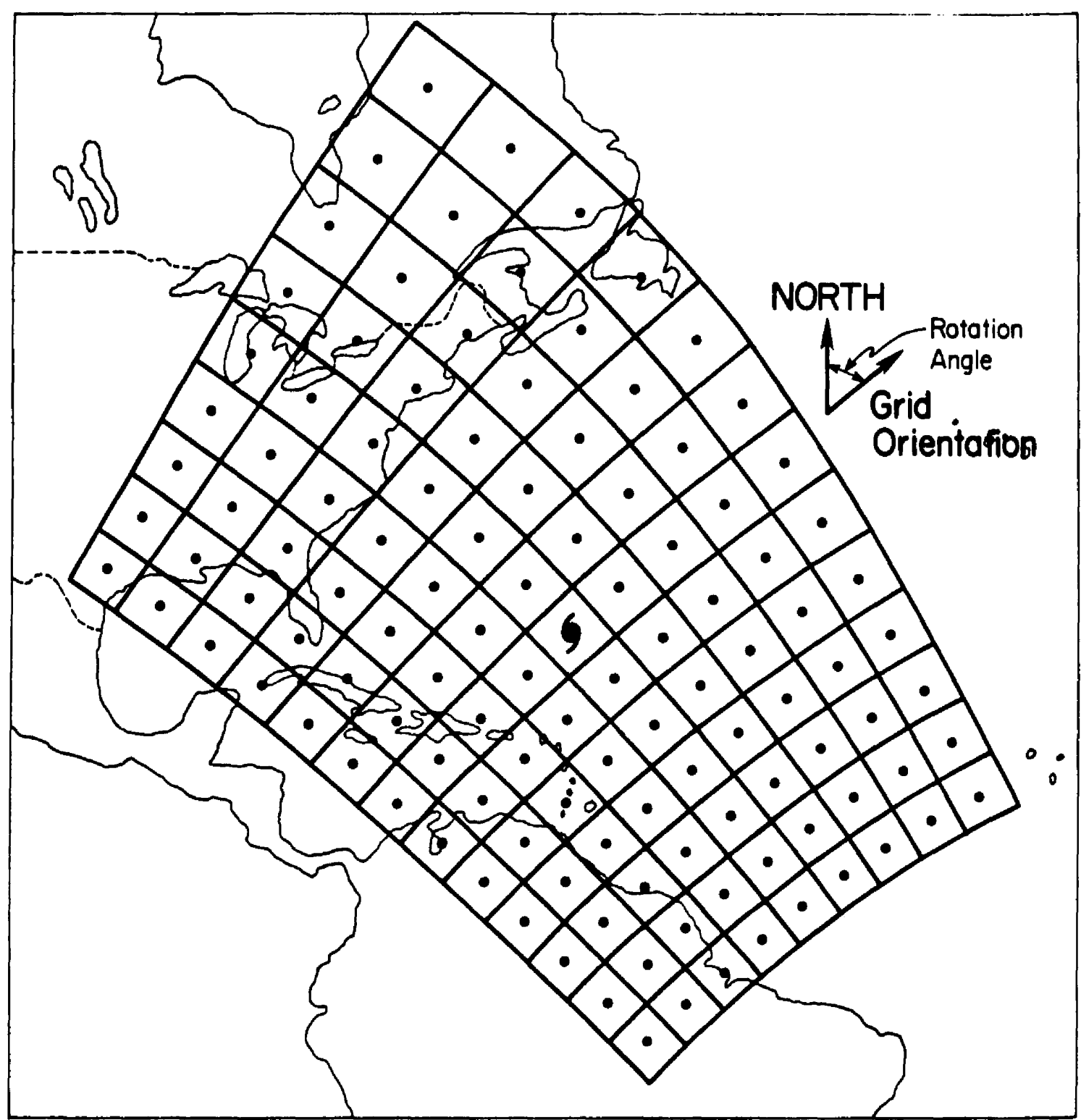

F18. 2.1. The Cartesian grid used in upper-tropospheric wind compositing. Th1s example is centered at $25.9 \% \mathrm{~N}, 60.8 \% \mathrm{~W}$ and has a rotation angle of $47^{\circ}$ from due north. The grid points (dots) are located at the center of the grid cells which are $500 \mathrm{~km}$ on a side. 


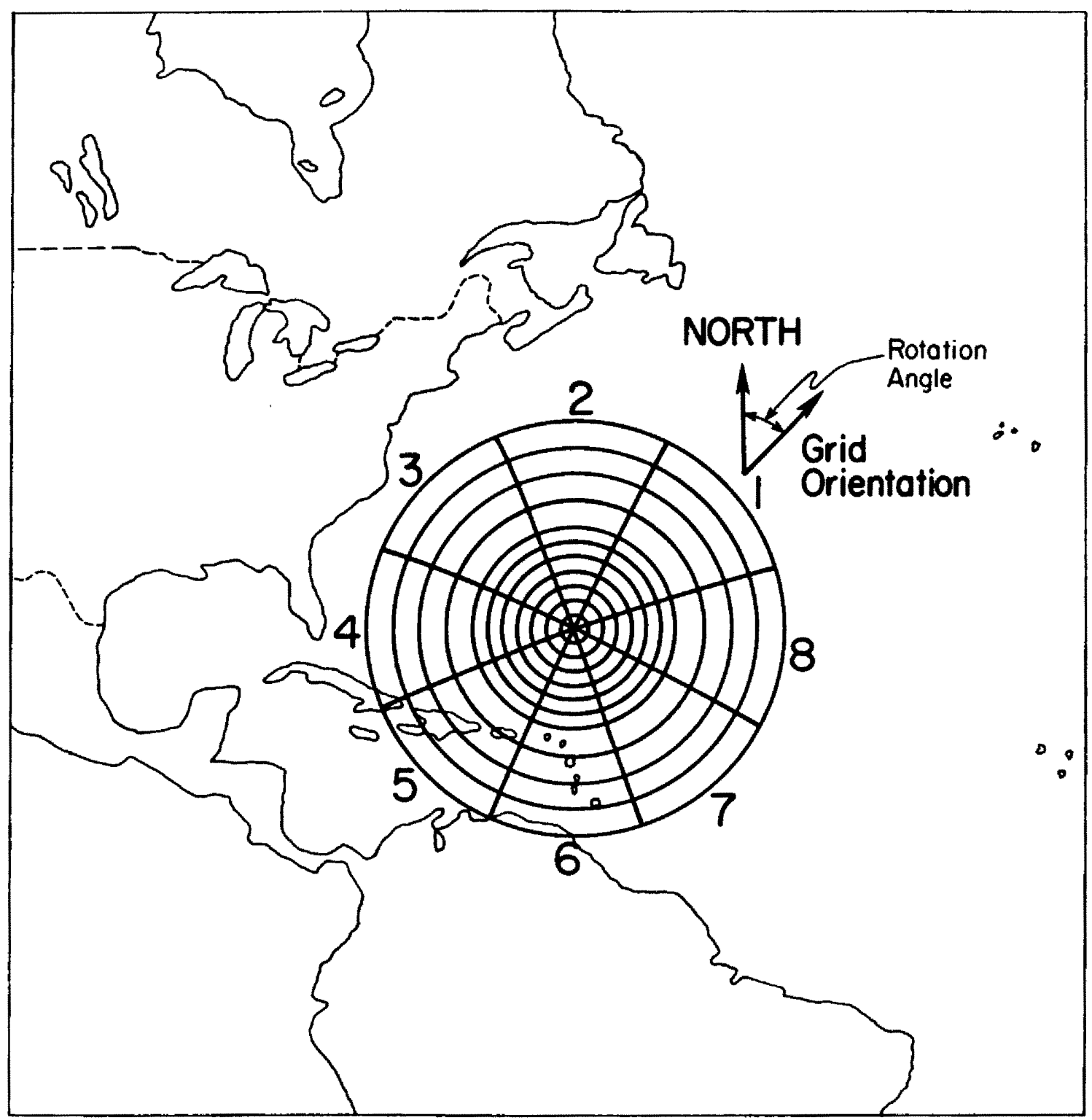

Fig. 2.2. The polar grid used in upper tropospheric wind compositing. The location and orientation are the same as for the Cartesian grid in F1g. 2.1. Octants are numbered counterclockwise, beginning with the octant centered on the specified grid orientation. 
observations and gridded analysis archived. The number of observations may vary, depending on the number of reports from commercial alrcraft and the amount and distribution of cirrus cloud, but it averages about 400, a roughly fourfold increase over rawinsonde data alone for the entire grid, and an order of magnitude increase for oceanic areas where hurricanes occur.

Efficient archiving of the raw data and analysis grids was begun in 1977, and it was decided to copy those tapes covering the times of significant Atlantic basin (including the Caribbean Sea and Gulf of Mexico) tropical cyclones from 1977-1983. The tapes for 1978 and the latter half of the 1982 seasons could not be read, but data covering the times of 37 Atlantic named tropical cyclones were extracted from the remaining years. The periods of record and cyclone occurrences are shown in Fig. 2.3 .

Several investigators (Bauer, 1976; Rodgers et al., 1979) have studied the properties of satellite-derived winds and have concluded that high level winds obtained from cloud motions compare well with winds measured by rawinsondes at the same level. The greatest source of error is incorrect assignment of the level of the satelitie derived wind. Cloud formation and dissipation can also introduce errors, particularly in low-level wind estimates, so short interval (7.5 minute) Imagery is best. The operational 30 minute interval is adequate for upper level winds because cirrus forms and dissipates more slowly. Especially because of the problen of level assignment, the combined wind set is undoubtedly prone to more errors, and those possibly of a systematic nature, than is a "conventional" data set consisting of 


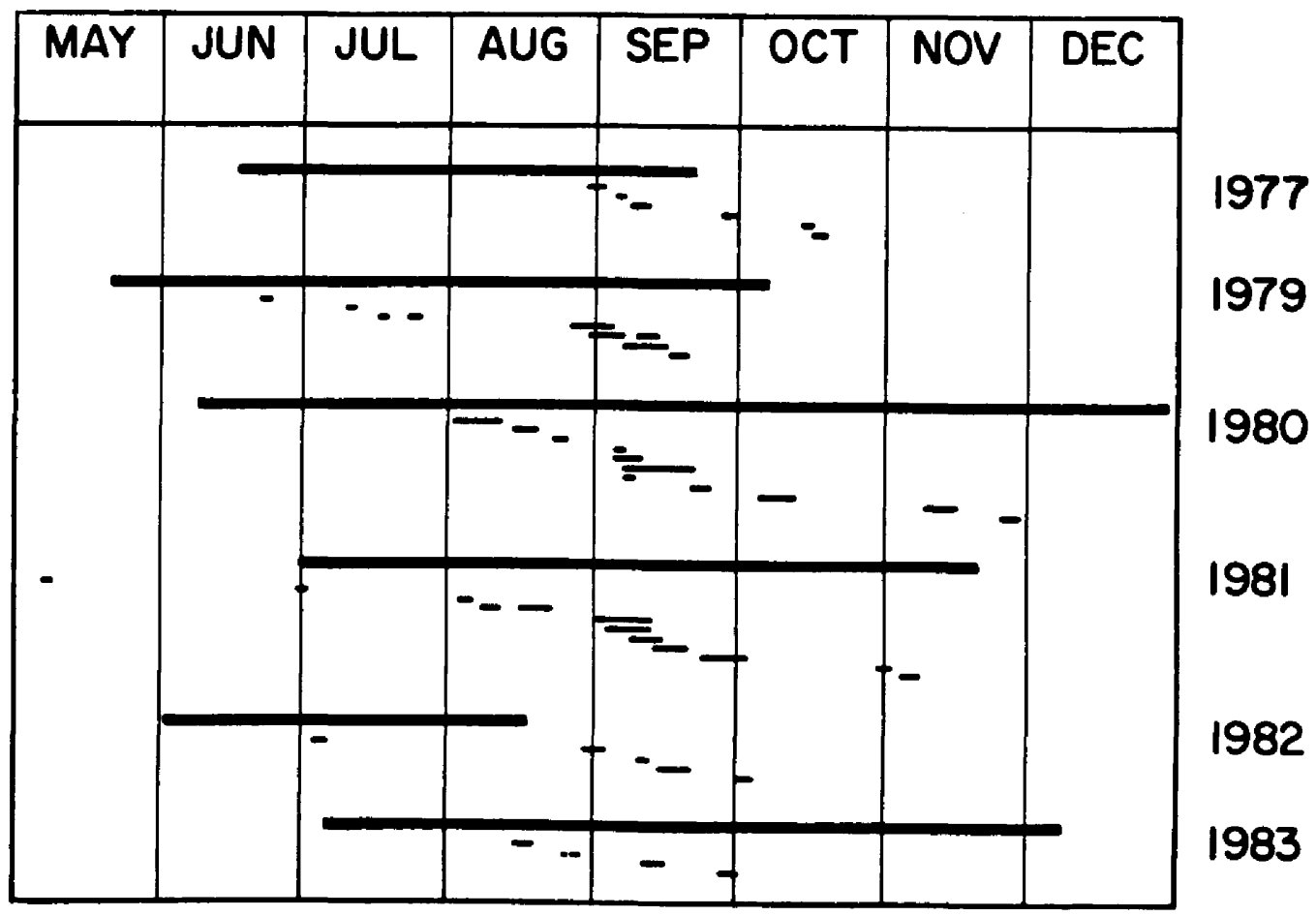

Fig. 2.3. Upper tropospheric wind set availability and Atlantic tropical cyclone occurrences, 1977-1983. The long lines indicate periods of data availability for each year, and the short lines are the times of named tropical cyclones for that year.

observations from one platform only. The recognized sources of error and their possible influence on the results are:

1. Rawinsonde reports at $200 \mathrm{mb}$ are probably below the level of maximum outflow from cumulus convection (and hence hurricanes) in the tropics. Composites of rawinsonde observations around hurricanes (Frank, 1977a) typically show maximum outflow at an average of $175 \mathrm{mb}$, although poleward outflow is lower (200 mb) than equatorward outflow (150 mb). At higher latitudes, $200 \mathrm{mb}$ is probably too high. The effect of using fixed-level data to measure the wind speeds in a vertically varying "outflow layer" probably tends to result in an underestimate of the speed.

2. Winds measured by commercial aircraft (altitudes of 200-300 mb) are likewise below the level of maximum outflow, particularly in low 
latitudes, and their use will result in an underestimate of the "outflow layer" wind as well. This effect is partially mitigated by the greater frequency of flights at higher latitudes where cloud tops and convective outflows tend to be lower.

3. Satelite-derived winds are obtainable only when clouds exists, so that the cloudy regions are oversampled. To the extent that cirrus cloud is associated with convective activity and outflow, the winds might be systematically stronger in cloudy regions, and the work of Wei and Gray (1985) using rawinsonde observations in clear and cloudy regions indicate that this is indeed the case. The oversampling should then, in a composite or statistical sense, yield a mean wind that is too strong. This effect will be greater if a small patch of cirrus is sampled repeatedly, yielding several highly correlated and largely redundant observations. Analysis of Individual cases should be unaffected, as cirrus motion has been shown to be a good representation of the ambient wind.

Estimating the combined effects of these errors is quite difficult as they are undoubtedly interrelated. For example, cirrus can travel long distances without sublimating (Merritt and Wexler, 1967), and does so only when it encounters pronounced subsidence. Under such subsident conditions, the air originating from a convective region at a pressure of $175 \mathrm{mb}$ may descend to $200 \mathrm{mb}$ or even lower, making the $200 \mathrm{mb}$ rawins and 200-300 mb aircraft winds actually more representative of the flow of interest in the cirrus-free areas than they would be in a cloudy region. Because the "ground truth" flow fields in the tropics are unknown, the only way to assess the effects of these errors is by preparing composites using a single observation type and comparing them. 
This has been done for the Gulf of Mextco which is ringed with rawinsondes, traversed by atroraft, and contains numerous tropical cyclones of tropical storm or greater intensity. Three composite upper-tropospheric wind flelds were prepared for these cyclones, using a) $200 \mathrm{mb}$ rawins, b) satellite cloud track winds, and c) aircraft winds. The results are shown for comparison in Fig. 2.4 (radial wind), Fig. 2.5 (tangential wind), and Figs. 2.6-2.8 (total flow fields). Radial profiles in Figs. 2.4 and 2.5 are computed using the polar grid (Fig. 2.2) and computing the average radial and tangential component for each grid cell. The octant averages are then averaged together for each belt to produce the belt means. Figures 2.6-2.8 are analyses of vector mean winds for the Cartesian grid (Fig. 2.1). As is expected, the aircraft winds give the smallest estimate of radial wind and the satellite winds the largest. Rawinsonde tangential winds are less anticyclonic than the nearly identical satellite and aircraft winds, probably due to variability of sampling location of the three wind types. Satellite winds are noticeably stronger than the two other types at points 1000-2000 km northeast of the center (Figs. 2.6-2.8) of the outflow jet where all three types of winds are found. Satellite winds are also stronger than rawins to the south of the cyclone and would probably be stronger than the aircraft winds as well, if there were any. Since the afrcraft winds come from the poleward side of the cyclone, they oversample the strong westerlies and introduce a negative bias to the symmetric mean tangential winds (Fig. 2.5).

2.5 Individual Case Analysis and Rotation Angle Determination This study focuses on the interaction between a developed tropical cyclone and its environment, as distinguished from the processes 


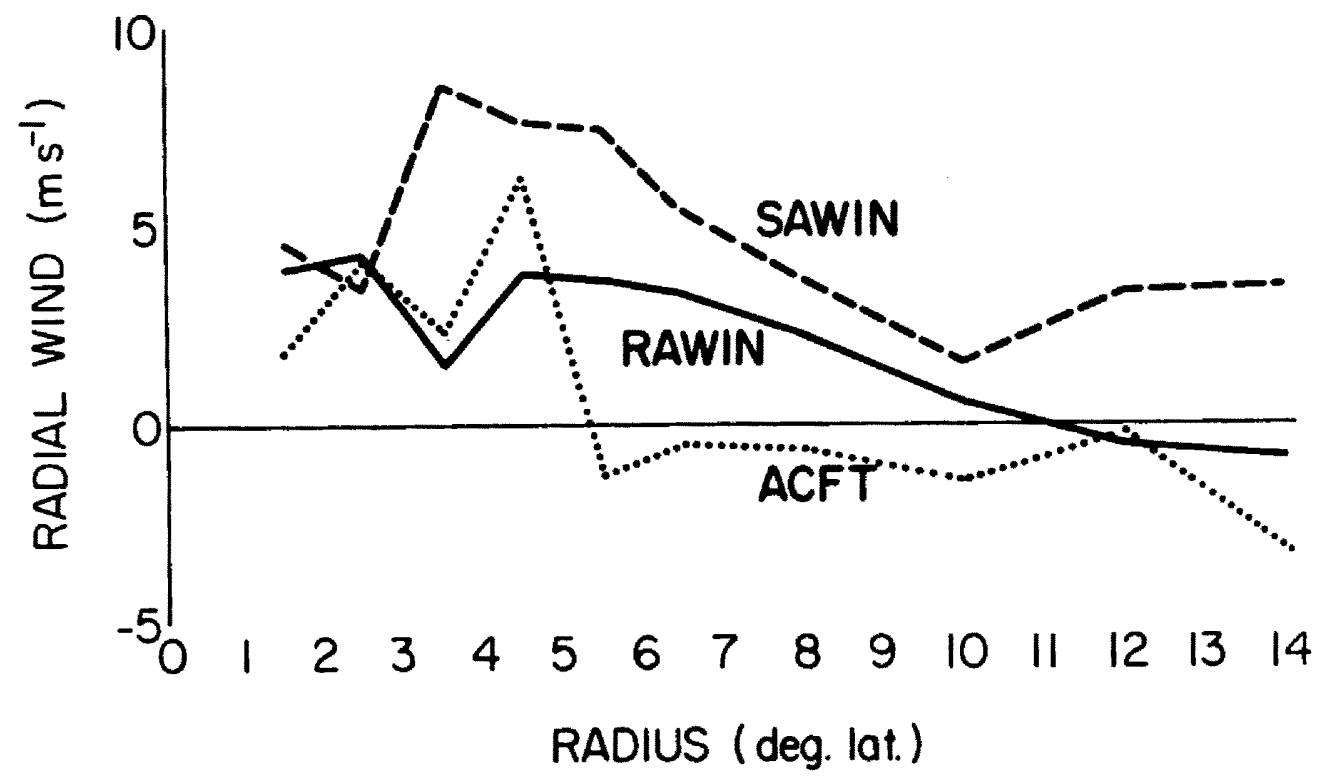

F18. 2.4. Radial profile of upper-troposphere radial wind for composite Gulf of Mexico named tropical cyclones. The solid line is the result using $200 \mathrm{mb}$ rawinsondes only, the dotted line is for commercial aircraft winds, and the dashed line for satellite derived cloud motion winds.

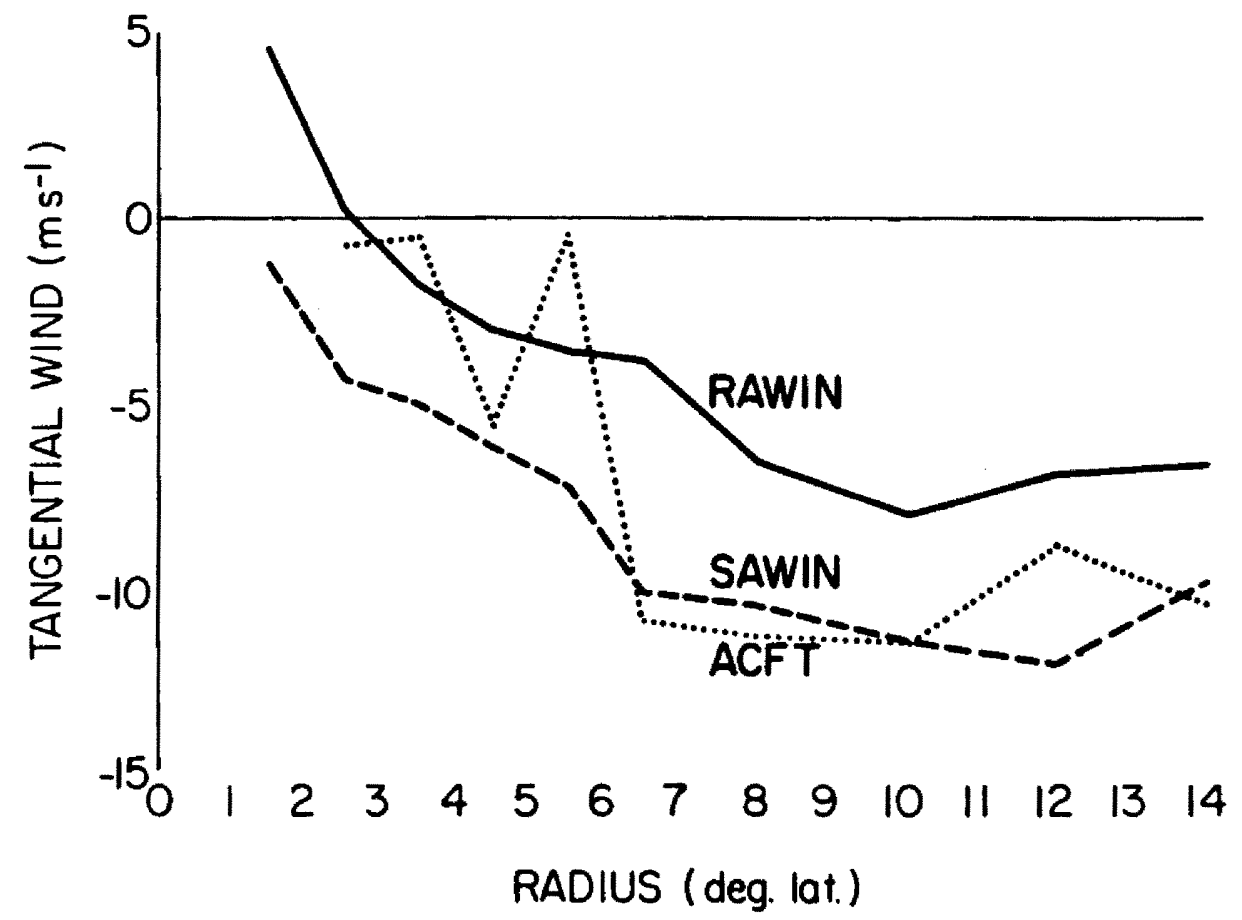

Fig. 2.5. Same as Fig. 2.4 except for tangential winds. 


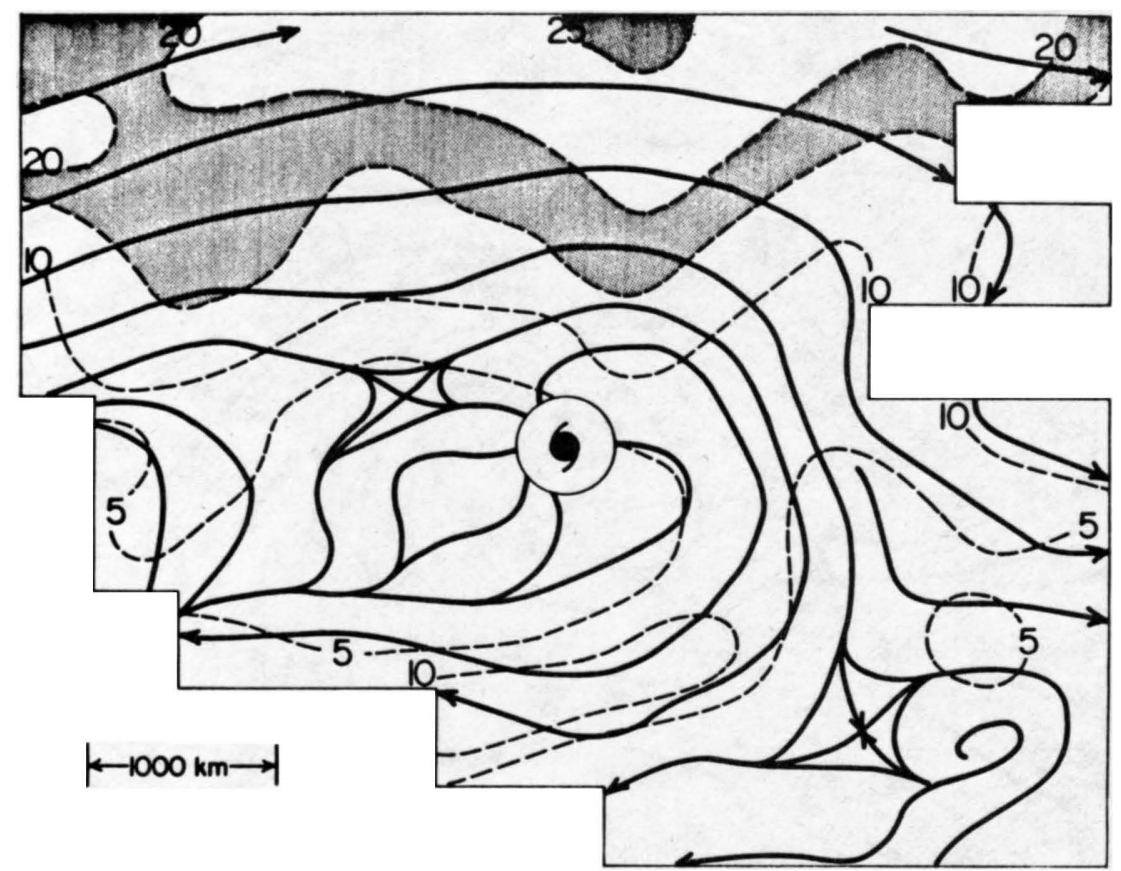

Fig. 2.6. Cartesian grid plan view of the resultant wind for the composite Gulf of Mexico named tropical cyclone. This composite contains rawinsonde observations only. Outline denotes limit of obseryation density of 5 per $(500 \mathrm{~km})^{2}$. Isotachs are at $5 \mathrm{~m} \mathrm{~s}^{-1}$ intervals, with shading beginning at $15 \mathrm{~m} \mathrm{~s}^{-1}$.

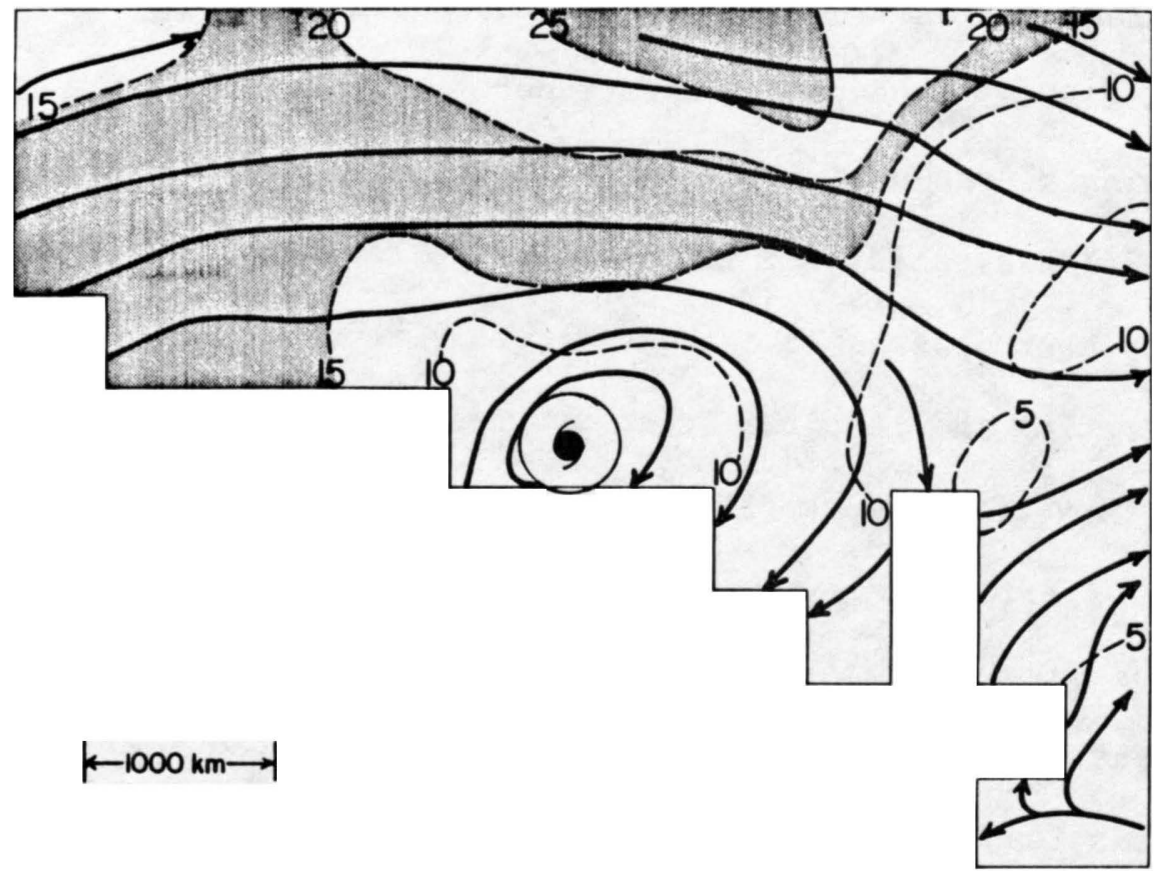

Fig. 2.7. Same as Fig. 2.6 except for commercial aircraft wind reports only. 


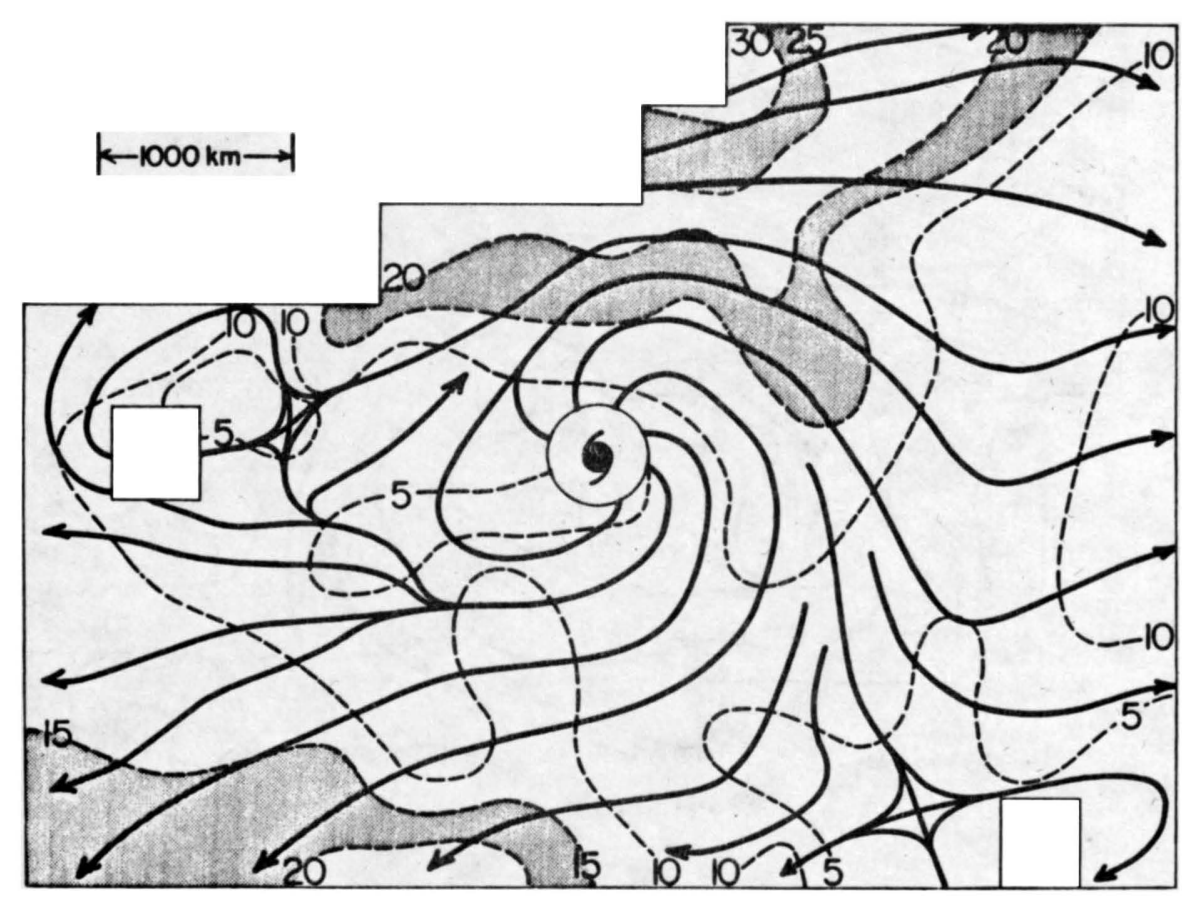

Fig. 2.8. Same as Fig. 2.6 except for satellite-derived wind observations only.

associated with tropical cyclone formation. The working hypothesis of environment-cyclone interactions of Holland and Merrill (1984) and Merrill (1984a) presupposes a warm-core structure on the synoptic scale possessing a near-neutral inertial stability in the upper troposphere. Merrill (1984a) also described the possible role of the existence of a small upper-tropospheric cyclone directly above the low-level vortex in concentrating the response to enviromental effects to the hurricane core. Many of the weaker systems operationally classified as "tropical storms" (particularly those which fail to reach hurricane intensity) lack one or both of these features and their intensity may be less dependent on upper-tropospheric effects than that of a hurricane. The processes leading to the formation of these weaker systems are different (Lee, 1985), being related more to low or mid-tropospheric effects. The 
study of Pacific reconnalssance data by Weatherford (1985) has indicated a similar happening; the tropical storms (which usually lack eyes) tend to have a maximum wind which is well related to the vortex strength as defined earlier. Typhoons, on the other hand, possess eyes and are characterized by much greater variability in intensity within a vortex of the same strength. Holliday (1979) Indicates that rapid

Intensification, the forecasting of which is the principal operational motivation for this study, only becomes a threat when the tropical cyclone has attained typhoon intensity and the eye-eyewall structure has already formed. The cases for outflow layer compositing were therefore selected from those tropical cyclones of hurricane intensity. Uppertropospheric wind set data were available for a total of 174 synoptic times (002 and 12Z) (benceforth called "positions") for 28 hurricanes of the period 1977-1983. These hurricane positions and data archival times were selected for the outflow jet compositing process.

Isolation of the outflow jet by coordinate rotation, which is the cornerstone of this investigation, requires that the orientation of the principal outflow jet be known for each hurricane position at each synoptic time. This requires analysis of each case. Figures 2.9-2.12 summarize the analysis procedure. For each of the 174 hurricane positions, the Cartesian grid described in section 2.3 is used (with a rotation angle of zero) to composite the raw wind observations for the appropriate map time (FIg. 2.9). The output is then a single wind vector for each grid point (provided the grid cell contained any observations) as shown in Fig. 2.10. These values are computed by simple vector averaging of all reports within a grid cell. Streamlines and isotachs at $5 \mathrm{~m} \mathrm{~s}^{-1}$ intervals are then subjectively added, and 


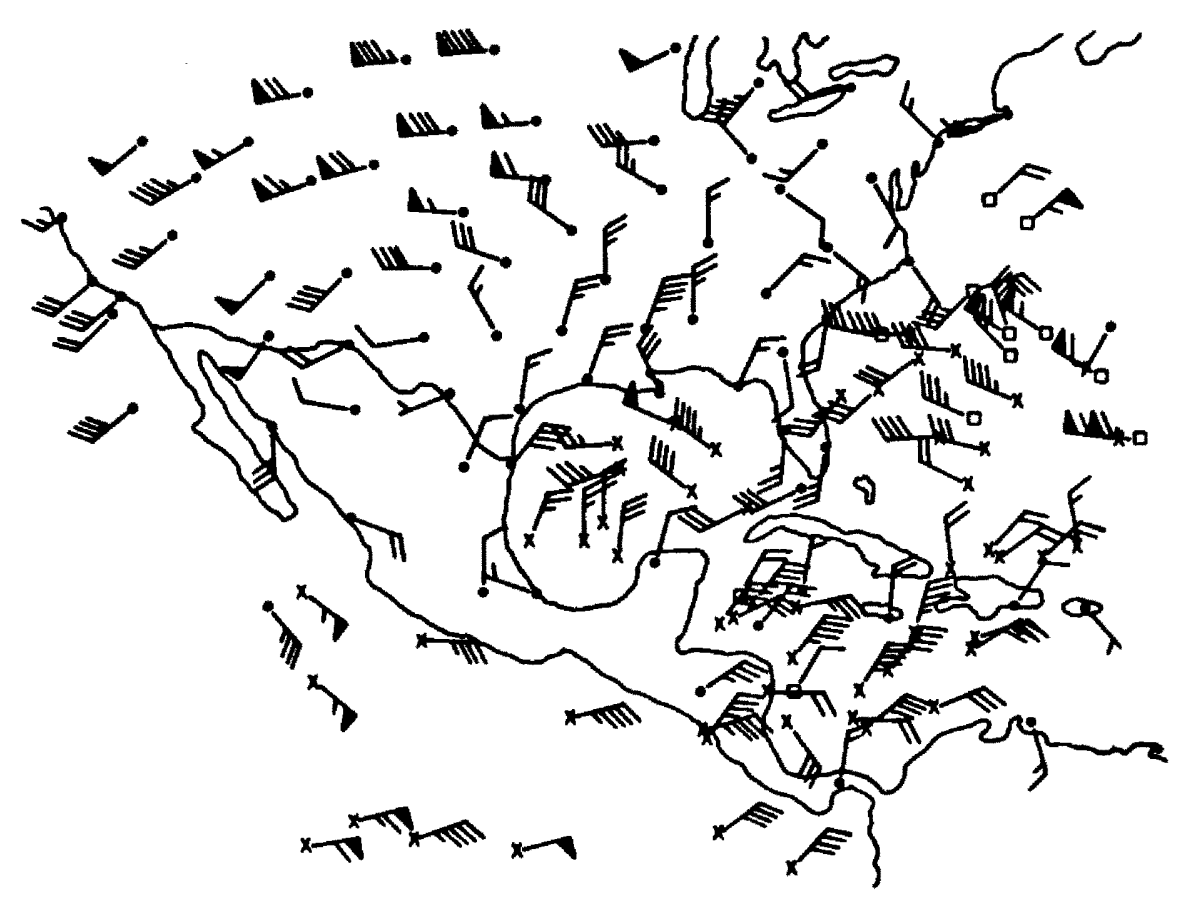

Fig. 2.9. Upper tropospheric wind reports for Hurricane Anita, 00 GMT 2 SEP 1977. Speeds are plotted in knots using standard windbarb convention. Dots are rawinsonde reports, squares are alroraft reports, and crosses are satelilte cloud track winds.

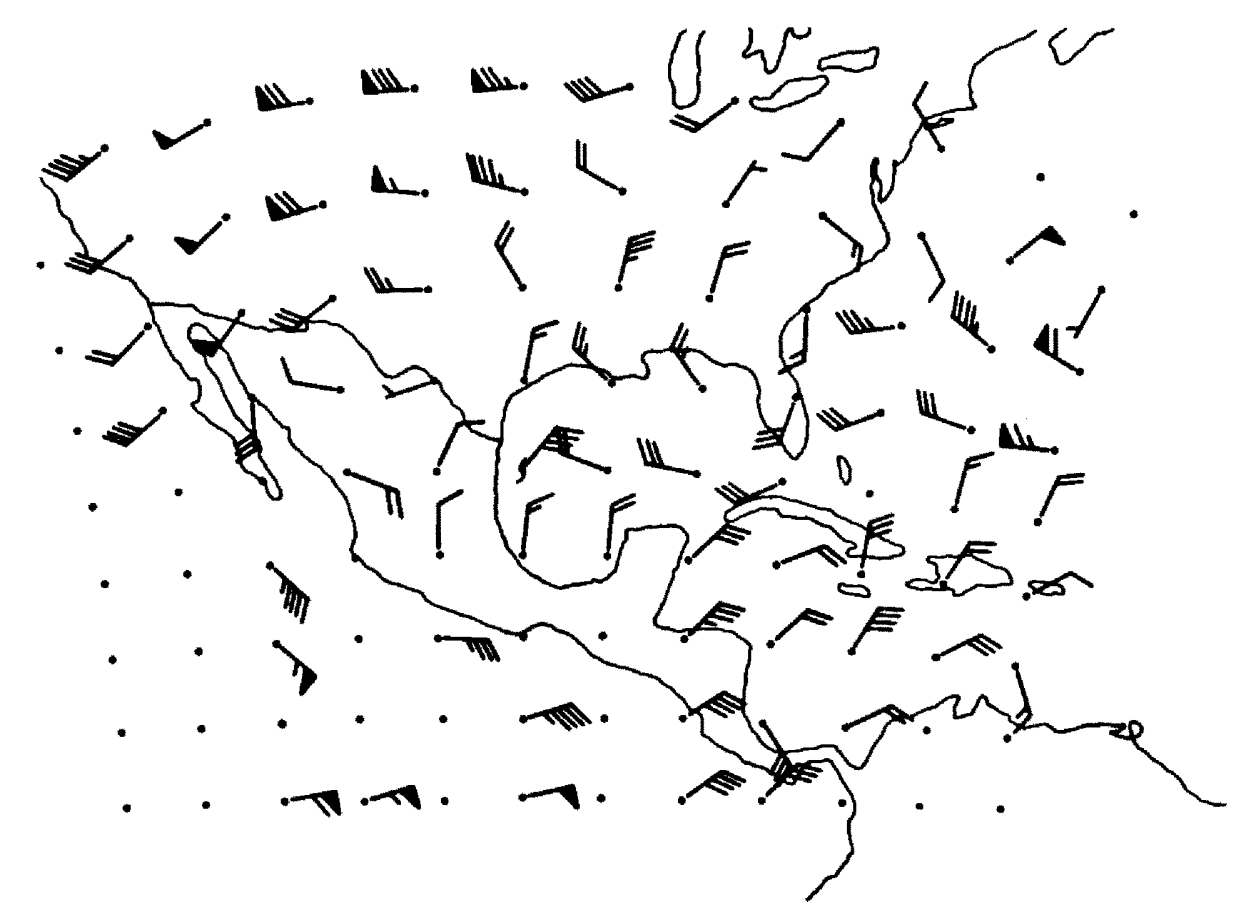

Fig. 2.10. Grid-point mean winds for Hurricane Anita, computed by vector average of the winds in Fig. 2.9 for each grid box. Wind plotting as in Fig. 2.9. 


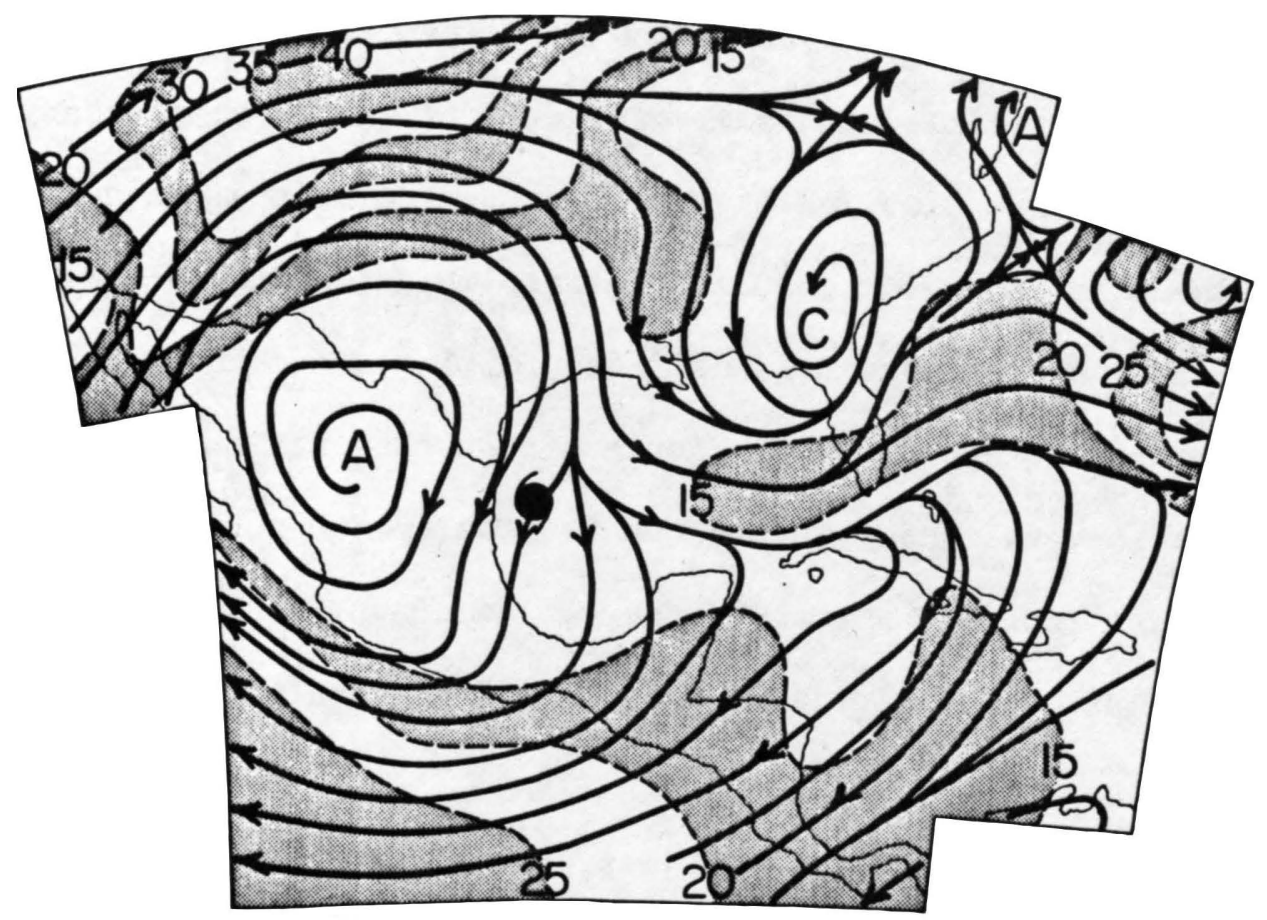

F1g. 2.11. Streamlines for Hurricane Anita, 00 GMT 2 SEP 1977 based on the grid point winds of Fig. 2.10. Isotachs are labeled in $\mathrm{m} / \mathrm{s}$.

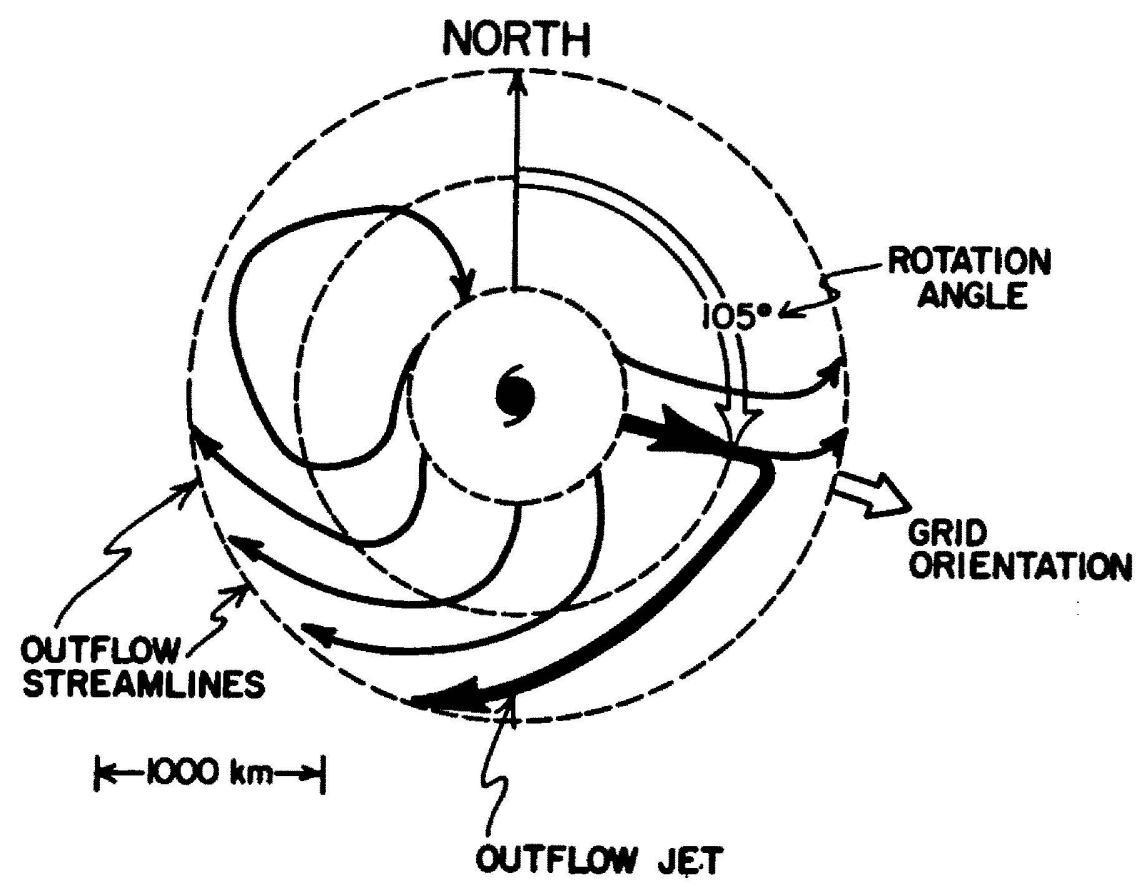

Fig. 2.12. Outflow streamlines, outflow jet, rotation angle, and grid orientation for Hurricane Anita, 00 GMT 2 SEP 1977 based on the wind analysis of Fig. 2.11. See text for a description of how these features are defined. 
conflicting reports checked against the navigated listing. Satellite photos are also avallable once daily for most of the hurricanes as an aid to analysis. The plotting of the average wind at the center of the grid region rather than its actual location (which varies randomly over the $500 \mathrm{~km}$ square) results in a position plotting error of up to $350 \mathrm{~km}$, with a mean error of about $170 \mathrm{~km}$ for a single observation, decreasing for multiple observations. This error is usually not noticeable and a smooth field fitting the average winds can be drawn, but occasionally the isotachs must be subjectively smoothed. The resulting wind analysis is shown in Fig. 2.11.

A systematic, though still subjective, procedure is then applied to determine the principal outflow jet, and this process is shown in Fig. 2.12. Ideally, the streamilne emanating from the eye wall is desired, but given the vertical and lateral mixing possible in the convectively active and inertially neutral flow over the hurricane and the lack of good observations within the central dense overcast, such a streamline cannot be determined. When the potential plotting position error of the wind reports is also considered, it can be concluded that any streamline emanating from a circle of $500 \mathrm{~km}$ radius centered on the hurricane could be consldered part of the "outflow" on the synoptic scale, and is defined as an "outflow streamline". These outflow streamlines from Fig. 2.11 (according to this definition) are shown in Fig. 2.12. Visual inspection of the analyses (and of the qualitative descriptions of Sadler, 1978) indicated that the "outflow jet" was associated with a synoptic-scale speed maximum, so that one outflow streamilne which was associated with the highest wind speed within $1500 \mathrm{~km}$ of the hurricane center is then designated the "outflow jet" (wide streamline in Fig. 
2.12). The angle from north at which this streamline crosses a $1000 \mathrm{~km}$ radius circle is the "outflow rotation angle" for compositing this case. The $1500 \mathrm{~km}$ radius restriction for defining the maximum wind speed was applied to the selection process to prevent an outflow streamline which was associated with high wind speeds only at very large radil from being designated the outflow jet over another streamline with a speed maximum more obviously assoclated with the cyclone. Figure 2.12 shows the outflow streamlines, wind speed maxima, outflow jet, and rotation angle for the example used in Figs. 2.9-2.11.

Occasionally, when multiple maxima existed in the 1sotach analysis, two outflow fets could be defined. The weaker of the two was accepted only if the speed attained within $1500 \mathrm{~km}$ was at least $15 \mathrm{~m} \mathrm{~s}^{-1}$. The stronger was designated the "primary outflow jet" and used to assign the rotation angle. If the two jets were of nearly equal speed, the time history of the flow was examined and the dominant jet on the next chart (12h later) designated as the primary.

During the subjective streamline analysis process, much was learned about the quality of the data and the general nature of the hurricane outflow layer. The data as obtained from NHC were extracted directly from the operational files, and several dozen "suspicious" grid-point mean winds were identified as not fitting the pattern being analyzed. The Individual observations makdng up each suspect mean wind were then located in the observation listings and the individual erroneous observation located and extracted from the data base. Analysis of individual cases also revealed that 30 positions ( 6 cyclones) operationally designated as "hurricanes" by NHC exhibited the cyclonic shear and/or vorticity aloft associated with subtropical cyclones. 
These "hybrids" were all classified as subtropical or extratropical earlier in their existence and were upgraded to hurricane status when their maximum winds exceeded $32 \mathrm{~m} \mathrm{~s}^{-1}$. The qualitative differences in outflow persisted throughout the hurricane stage, so these systems were extracted from the position file and composited separately. Their characteristics will be discussed and compared with those of the "tropical" hurricane in Chapter 3.

The only "new" aspect of the composite procedure, the outflowbased coordinate rotation, was tested using two comparison runs; an outflow-rotated (OROT) run using the outflow rotation angle for each position, and a fixed rotation run (FROT) using a rotation angle of $47^{\circ}$ clockwise from north (mean rotation angle of OROT) for all positions. Figures 2.13 and 2.14 show the resultant winds on the Cartesian grid. Considering that the coordinate rotation is chosen so that the outflow axis crosses $1000 \mathrm{~km}$ radius towards the front of the grid (star on OROT plot in Fig. 2.13) it is not surprising that this point shows the largest resultant wind speed $\left(21.5 \mathrm{~m} \mathrm{~s}^{-1}\right)$ and the greatest radial wind. To the northwest lies a confluent westerly stream, while to the southeast lies a synoptic-scale anticyclone and, at a distance of 2500 $\mathrm{km}$ an upper-level trough. Figure 2.14 shows the FROT composite resultant winds. The westerlies poleward of the cyclone are less confluent and about $5 \mathrm{~m} \mathrm{~s}^{-1}$ stronger, the anticyclone to the southeast is less distinct, and the "outfiow jet" is now no longer a separate entity, but merely an equatorward elongation of the strong westerlies with only a slight relative maximum in resultant speed, displaced $500 \mathrm{~km}$ nor thwestward from the maximum wind point in the OROT composite. The upper trough far to the east is still present. 


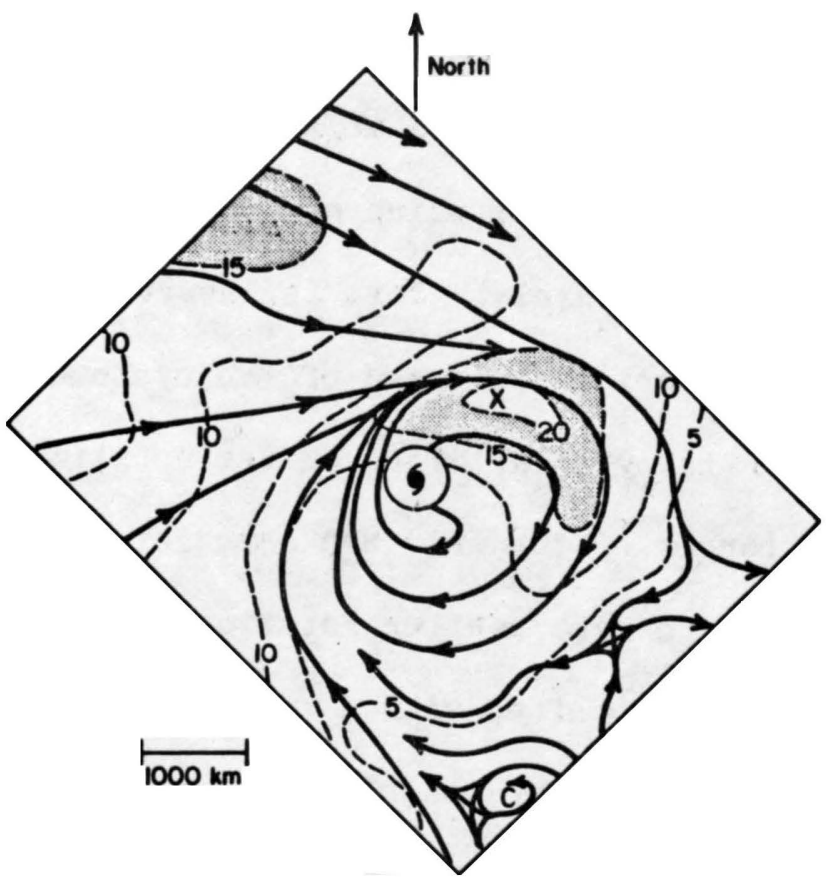

Fig. 2.13. Resultant winds $\left(\mathrm{m} \mathrm{s}^{-1}\right)$ for the outflow rotated (OROT) composite of Atlantic hurricanes. The Cartesian grid was oriented such that the principal outflow jet in each case passed through the point marked with a $X$. This point was, in the mean, rotated $47^{\circ}$ from due north.

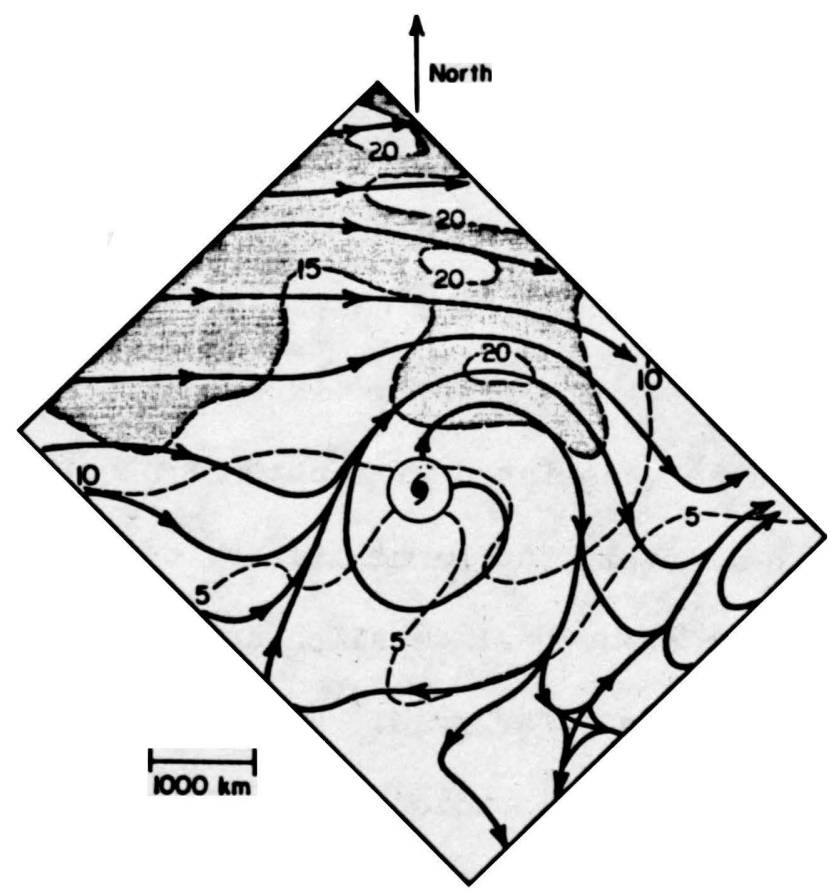

F1g. 2.14. Same as Fig. 2.13 except in "fixed rotation" (FROT) coordinates, using a rotation angle of $47^{\circ}$ (the mean rotation angle of $\mathrm{Fig} .2 .13)$ for each case, regardless of outflow jet location. 
The goal of coordinate rotation, isolation of the outflow jet as a distinct entity, is achieved, and the closed anticyclone (seldom seem clearly in rawinsonde composites, which are methodologically equivalent to FROT) is also depicted clearly. This is, however, at the expense of a distortion in the westerlies poleward of the cyclone. The strong confluence may be partially an artifact of the rotation process. The winds in the upper corner of the grid are associated with low-latitude hurricanes, which tend to have smaller rotation angles than the mean of $47^{\circ}$. A true west wind composited with a zero rotation angle thus appears more like a northwesterly when plotted on a grid which has been rotated $47^{\circ}$. Winds along the lower right edge of the grid tend to come from higher-latitude hurricanes with larger rotation angles, so westerlies, when composited and plotted, are distorted to appear somewhat like southwesterlies. Similar distortions can occur in rawinsonde composites as a result of an irregular station distribution. Much of the confluence is probably "real" and representative of the typical case, as other rotated composites of hurricanes in a more limited latitude range also indicate confluence in the same area. Holland and Merrill (1984) and Merrill (1984a) hypothesized that such a confluent flow would be associated with upper tropospheric frontogenetic processes acting to maintain the baroclinfcity of the outflow jet.

Some of the finer structural details can be resolved by examining the composites made on the polar grid. Figure 2.15 is the OROT polar composite. Structural features noted on the Cartesian grid in Fig. 2.13 are still evident, and slight evidence of cyclonic turning is apparent at inner radil. Flgures $2.15-2.18$ have been analyzed to reflect the existence of a cyclonic vortex in the upper levels, as has been found by 


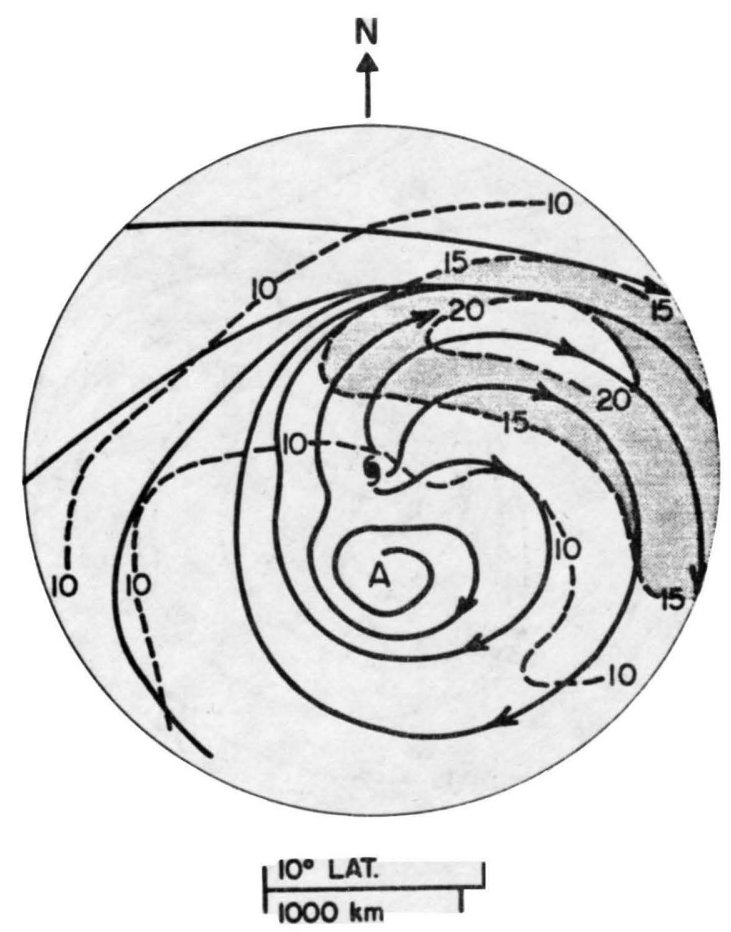

Fig. 2.15. Resultant winds $\left(\mathrm{m} \mathrm{s}^{-1}\right.$ ) for the outflow rotated (OROT) composite of Atlantic hurricanes as in Fig. 2.13 except calculated and plotted on the polar grid.

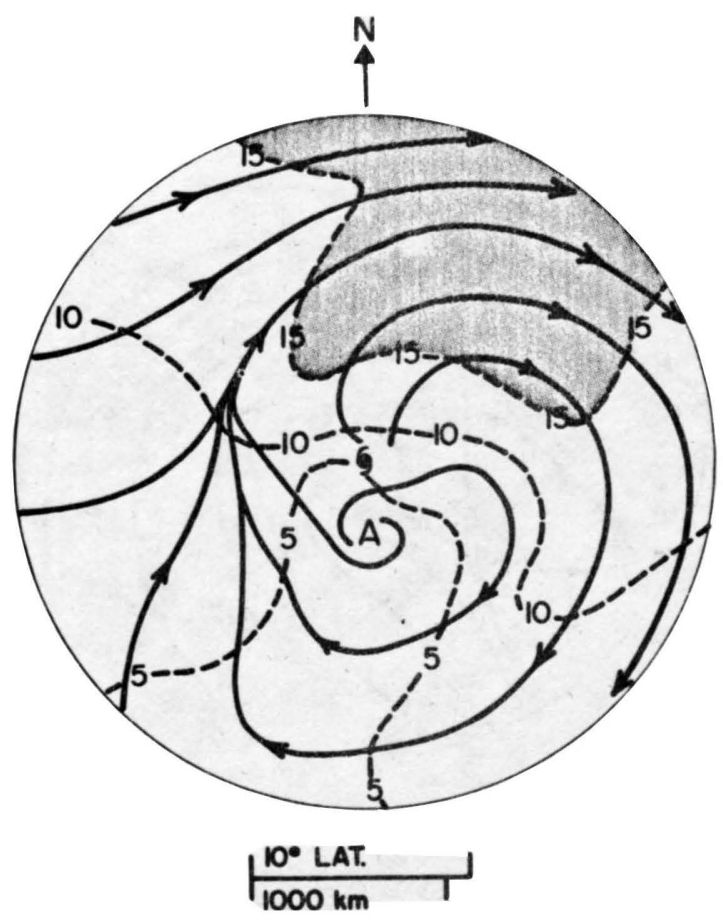

F1g. 2.16. Resul tant winds $\left(\mathrm{m} \mathrm{s}^{-1}\right)$ for the "fixed rotation" (FROT) coordinates as in Fig. 2.14 except calculated and plotted on the polar grid. 


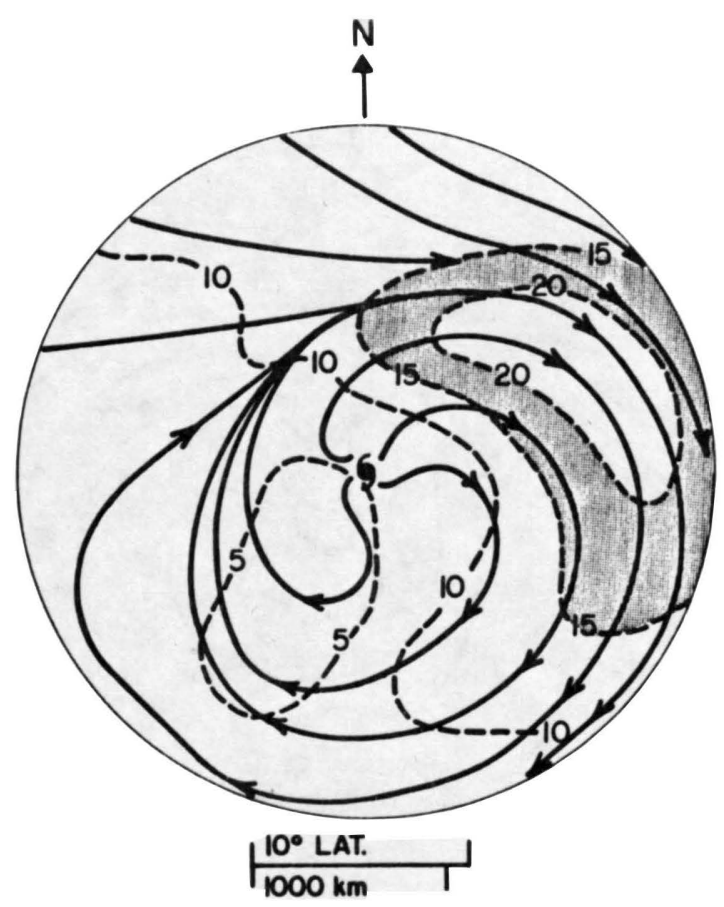

Fig. 2.17. Outflow rotated coordinate resultant winds with cyclone motion subtracted before compositing (MOT-OROT).

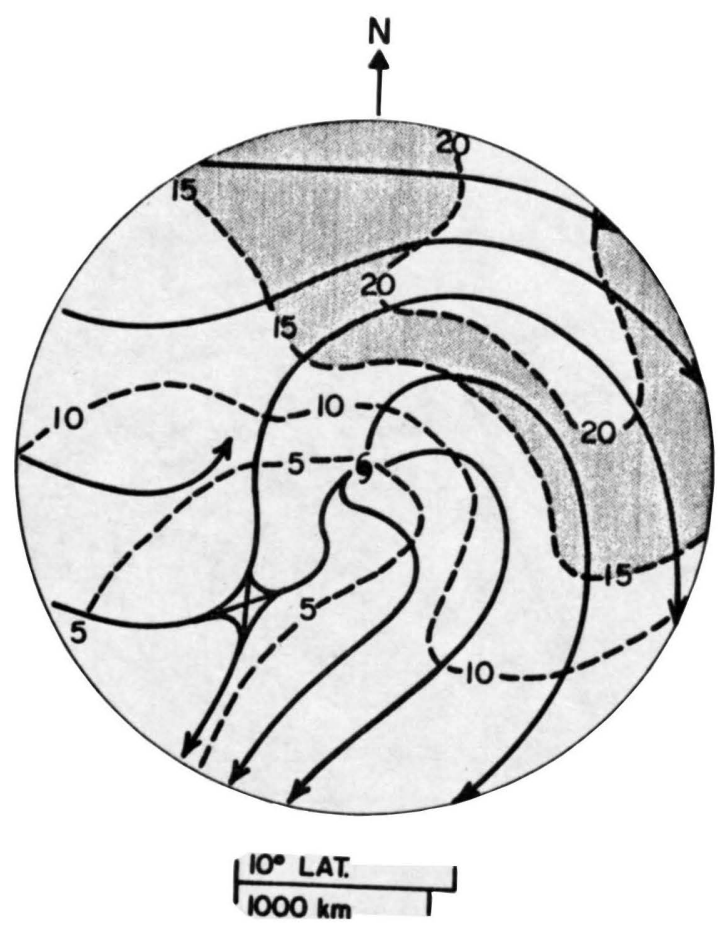

Fig. 2.18. Fixed rotation coordinate resultant winds with cyclone motion subtracted before compositing (MOT-FROT). 
upper-tropospheric aircraft penetrations made in a few hurricanes prior to 1966. Figure 2.16 is the polar representation of the FROT flow. As in the Cartesian grid (Fig. 2.14), the FROT composite indicates a weaker and less distinct outflow jet, less pronounced confluence to the northwest, and a NE-SW elongation of the anticyclone to the southeast of the vortex. Still another representation can be had by subtracting the vector motion of the cyclone from each wind observation during the compositing process to produce the mean flow relative to the moving cyclone. Figure 2.17, MOT-OROT, shows a slightly stronger outflow maximum, the closed anticyclone is no longer evident, and the cyclonic core region is somewhat more symmetric and with weaker wind speeds, Indicating that the upper-tropospheric shearing flow relative to the moving vortex is less than the actual wind of Fig. 2.15. Similar changes occur from FROT to MOT-FROT (F1g. 2.18).

The divergence of the composite flow is similarly affected by the outflow-rotation method with the OROT divergence field (Fig. 2.19) being more asymmetric than the FROT divergence field (Fig. 2.20). Note the strong convergence in the confluence zone to the northwest of the cyclone, and the second center of convergence associated with the exit region of the outflow jet to the east at about $1500-2000 \mathrm{~km}$ radius.

The outflow rotation method has been shown to resolve explicitly the asymmetric jet structure of the outflow layer. The remainder of this Chapter describes the IImitations of this technique and a suggested updrade for future use. In Chapter 3 the method is then applied to the description of the variety of outflow patterns of Atlantic hurricanes and the examination of outflow differences between intensifying and non-intensify ing hurricanes. 


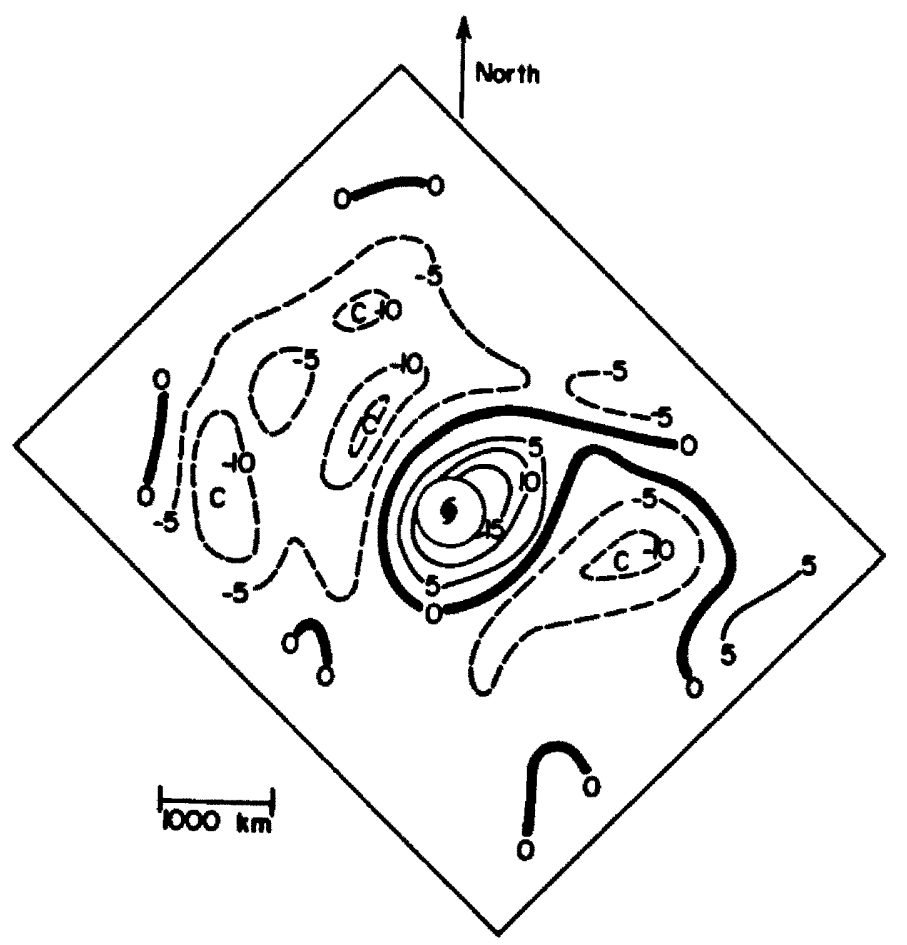

Fig. 2.19. Divergence (units of $10^{-6} \mathrm{~s}^{-1}$ ) for the OROT wind field composite shown in Fig. 2,13 .

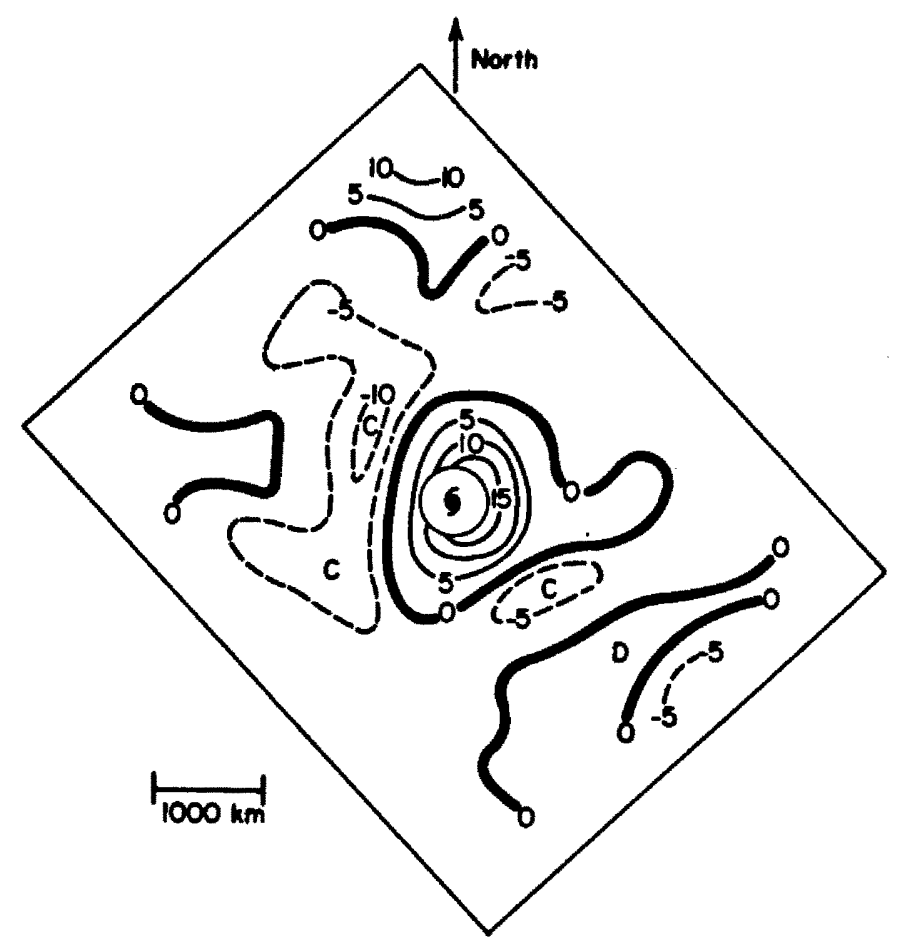

F1g. 2.20. Same as Fig. 2.19 except for the FROT wind field shown in Fig. 2.14 . 
2.6 Discussion of the Composite Method and its Limitations

This chapter describes the combined upper-tropospheric wind set and the rotated-grid compositing method used to study the structure of the hurricane outflow layer. This approach is a natural outgrowth of the rawinsonde compositing method used by William Gray and his graduate students for over a decade. The principal innovations are: 1) the use of a high-density data set with pre-analysis of the fields on a caseby-case basis, and 2) composite processing of cases as a function of flow characteristics. Previous (rawinsonde) compositing studies relied upon archived cyclone parameters (location, motion, intensity, size) to stratify the cases into samples for compositing and to navigate the data relative to the feature of interest (1.e., the hurricane center). This study adds a flow-based parameter, the outflow rotation angle, to the navigation process, and a flow-based description, outflow pattern type, to the stratification process. The result is improved resolution of asymmetries at and near the radius where the rotation angle is defined. Experience with the technique has revealed some deficiencies, and though a descriptive study of the outflow structures of different classes of systems (weaker tropical cyclones, eastern Pacific hurricanes, etc.) using the existing data set and software could be profitable, additional studies of outflow layer kinematics should be preceded by an upgrade of the technique as described below.

The most serious deficiency of the methodology at present is the averaging procedure used to determine the mean value of a parameter in a given grid box. The procedure, lifted directiy from the rawinsonde compositing method, is to take a simple arithmetic mean. With a rawinsonde spacing of 500-1000 km, this means that no more than one 
rawinsonde per map time would fall in each grid cell. With satellite and aircraft winds, observations are frequently taken less than $100 \mathrm{~km}$ apart, and tend to occur in bunches. Because (for synoptic scale structures resolveable by compositing) the wind at a point is highly correlated with the wind $100 \mathrm{~km}$ away, these multiple observations are redundant. Five observations at one synoptic time in one grid cell are not really five observations, but one observation taken five times. The difficulty arises in statistical treatment of the composite means, since the assumption of "Independent observations" comprising the sample in each grid box is clearly violated. Straightforward application of a Ttest, for example, would yield a confidence interval on the mean which is unrealistically small.

The recommended improvement is the use of some sort of objective analysis as a pre-processor on the raw wind data. The simplest would be to use a Cressman-type (Cressman, 1959) objective analysis in the composite program in which the raw data points for each synoptic time within a specified radius of a rotated-grid composite point would be averaged (using a welghted sum) and the resulting average used as a single "data point" in the composite. Data vold areas would yleld no observations within the influence radius of any grid point and would make no contribution to the composite. It would also be possible in such a method to include a variable weighting factor depending upon the type of observation (cloud track wind, rawinsonde, or aircraft). Figure 2.21 shows schematically how wind data for a single map time would be preprocessed before compositing. Grid point 1 would contribute one "observation" to the composite; the observation consists of the weighted average of the six winds within the influence radius. Point 2 


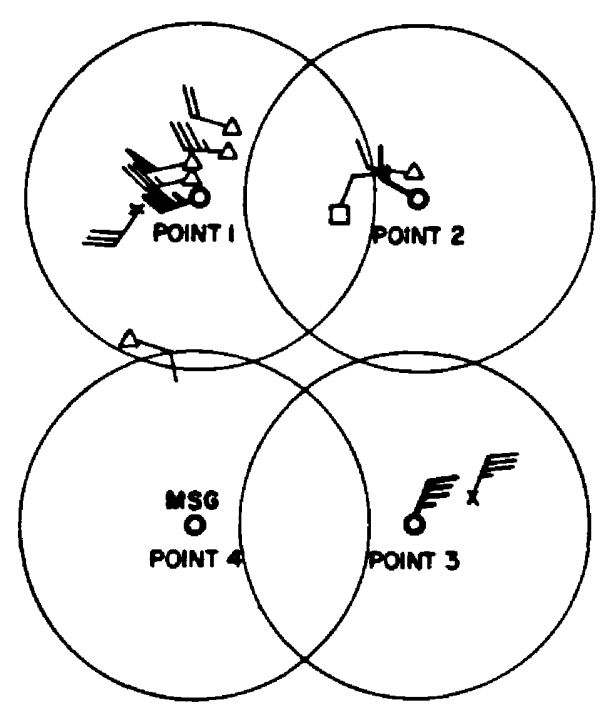

$\begin{array}{ll}\text { - POINT ON ROTATEO GRID } \\ \Delta & \text { SATELLITE WIND } \\ \times & \text { RAWINSONDE } \\ \square & \text { AIRCRAFT REPORT }\end{array}$

F1g. 2.21. Schematic of the uprated composite method suggested for future studies using the combined upper-tropospheric wind set. A complete description is given in the text.

would also contribute one "observation" based on two points - note the heavier welght given to the cloud-motion wind. The single wind within the influence radius of point 3 would be included "as is", and the fourth grid point would be "missing" and make no contribution to the composite. Note that any grid point in the composite could contain no more "observations" than the number of tropical cyclone positions with which the composite was made.

Statistical comparisons between composites using such a method, though still subject to the serial correlation between synoptic times (Neumann, et al., 1977), would be much more conservative than those based upon the present averaging method. Development of the optimum influence radil, distance welghting factors, and observation type weighting factors would require additional research. Only then could 
"production" runs for outflow layer studies be resumed. Other deficiencies of the wind observations themselves have been previously described in section 2.4 . 


\section{OBSERVATIONS OF THE UPPER TROPOSPHERIC ENVIRONMENT}

The previous chapter describes the high-density combined wind set and the compositing method used to process it so as to retain the asymmetric structure of the outflow layer. This chapter describes the application of this data set to the study of outflow layer structure and its relationship with hurricane intensity change. The first section describes the different outflow patterns which occur in the Atlantic basin. These patterns are determined by the synoptic scale flow features surrounding the hurricane. Generally, there are no "Intensifying" or "nor-intensifying" patterns. To see the outflow layer differences which are associated with intensity tendency, it becomes necessary to consider the effect of ocean temperature (section 3.2) and make composites based specifically on intensity trend (section 3.3). The changes in outflow structure as a hurricane intensifies and then begins to $f i l l$ are described in section 3.4 , and a descriptive summary of the observed outflow differences between intensifying and non-intensifying hurricanes is given in section 3.5 .

\subsection{Outflow Pattern Types for Atlantic Hurricanes}

During the individual case analysis process required to determine outflow rotation angles (section 2.5 ), it became apparent that several distinct upper-tropospheric synoptic patterns were associated with Atlantic hurricanes. This finding supplements the work of Chen and Gray (1985) who qualitatively defined synoptic patterns of outflow on a global basis using the FGGE III-b data set, covering a one year period. 
Previous investigators bound by data and/or processing limitations to a small number of cases had tended to concentrate on a single pattern type (Ramage, 1974; Sadler, 1978; Simpson, 1971) or on smaller scales where the pattern differences are not so obv1ous (Black and Anthes, 1971). This section describes eight pattern types and presents uppertropospheric flow composites of each. The usefulness of the patterns in forecasting and further research will then be discussed.

Subtropical origin. Of the 174 hurricane positions available, 30 fell into the "subtropical" pattern. The specifications for this pattern have previously been stated in part (section 2.5) as prior classification of the system as an extratropical or subtropical storm. An additional condition, that of cyclonic curvature and/or shear al of averaged over a $1000 \mathrm{~km}$ radius from the surface center, has been added. Subtropical-origin hurricanes tend to intensify slowly and, in the limited sample available, have a lower bound on pressure at about 970 mb. This may be due to the high latitudes (usually poleward of $30^{\circ} \mathrm{N}$ ) and cooler sea-surface temperatures at which they are usually found, or to the overall "Inefficiency" of the pattern. The latter is thought to be the case since several other (non-subtropical origin) hurricanes (Gladys 1975, Ella 1978, Debby 1982) have traversed the same area as the most intense subtropical pattern system (Emily 1981) while reaching lower minimum pressures. Subtropical-pattern systems tend to be much larger than average (Merri11, 1984) and those investigated by reconnaissance are found to have a relatively weaker maximum wind than is typical for their observed minimum pressure. These subtropical pattern hurricanes often have a small, symmetric central dense overcast (CDO) with a pronounced front-like banding feature. The overall 
appearance is not unlike that of an occluded baroclinic low with a "hurricane" in the middle. Another type of "subtropical storm" (Hebert and Poteat, 1975) is a mesoscale vortex within a predominantly linear cloud band, an example of which struck the Tampa Bay area on 18 June 1982 (Clark, 1983) and caused appreciable wind damage and local flooding. These tend to be small and quick-moving and of less than hurricane intensity. None of these systems are contained in the composite data sets in this paper.

The Cartesian grid composite wind field (OROT) for intensifying subtropical-origin hurricanes is shown in Fig. 3.1. A comparison with Fig. 2.13 (OROT resul tant winds for all hurricanes) reveals some general similarities; both have a ridge axis to the southeast and a trough far to the east-southeast. Differences outweigh the similarities as the subtropical-origin pattern indicates a mean trough over the low-level vortex, cyclonic curvature and shear, and strong upper-level winds over the center. The center is located to the left rear, rather than the right rear, of the outflow speed maximum.

Subtropical jet. The subtropical jet pattern is similar to that described by Ramage (1974) in connection with several intensifying typhoons in the South China Sea. These cases tend to occur early and/or late in the season. Figure 3.2 shows the Cartesian grid composite. The outflow consists of a wind maximum emanating from the cyclone to the northeast, where it merges with a very strong (scmetimes $260 \mathrm{~m} \mathrm{~s}^{-1}$ in Individual cases) westerly subtropical jet stream. A trough is of ten present northwest of the cyclone as well. The pattern is defined by an outflow streamline from the nor theast quadrant which remains at 


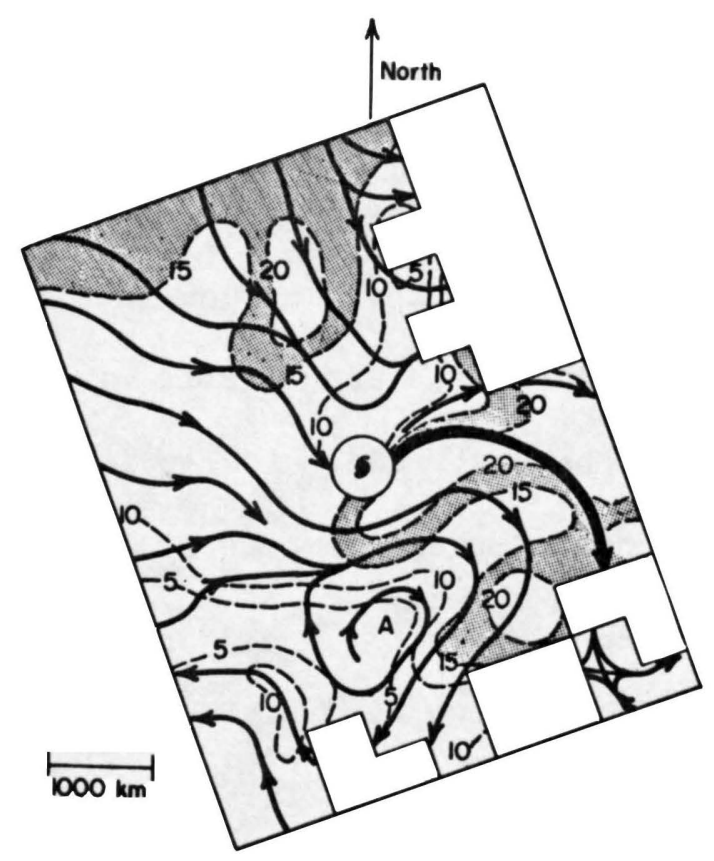

Fig. 3.1. Resul tant upper-tropospheric winds $\left(\mathrm{m}^{-1}\right)$ for the outflowrotated (OROT) composite of subtropical origin hurricanes. The indicated "north" corresponds to a grid rotation equal to the mean outflow rotation angle for all positions in the composite.

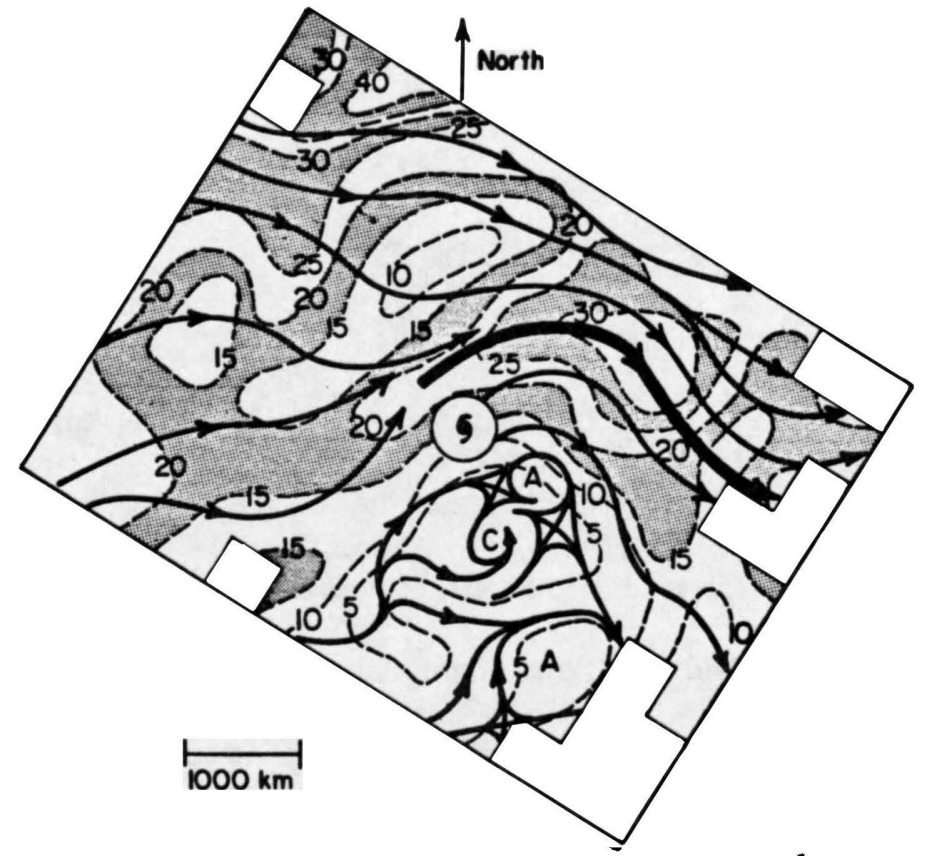

Fig. 3.2. Resultant upper-tropospheric winds (m $\mathrm{s}^{-1}$ ) for the outflowrotated (OROT) composite of hurricanes having a subtropical jet outflow pattern. The indicated "north" is defined as in Fig. 3.1 . 
a higher latitude than the cyclone out to $2000 \mathrm{~km}$ eastward, and a surface cyclone location more than $500 \mathrm{~km}$ poleward of the principal east-west ridge axis. Baroclinity in the mid-troposphere is apparently present in this pattern, as these cyclones are generally moving poleward ahead of the westerly trough, thus indicating a midlevel southerly component to the environmental steering flow. Intensification may be relatively rapid but is often limited to a short period of $24 \mathrm{~h}$ or less as the poleward motion soon carries the cyclone under the upper westerlies where it is destroyed by vertical shear.

IUTT pattern. The definition of the Tropical Upper Tropospheric Trough (TUTT) pattern is identical to that for the subtropical jet pattern, except that the cyclone is now located equatorward, or less than $500 \mathrm{~km}$ poleward, of the principal east-west ridge axis. This is the pattern described by Simpson (1971) and Sadler (1978) for lowlatitude $\left(\leq 20^{\circ} \mathrm{N}\right)$ Atlantic hurricanes and Pacific typhoons respectively. This pattern (Fig. 3.3) shows outflow again to the northeast, along the southeastern margin of a trough or shear line. This trough is a semipermanent feature of the central Atlantic (and Pacific) during summer, and the baroclinicity associated with the TUTT and associated cold lows is typically more shallow than that of midlatitude troughs, being largely confined to the upper troposphere (Kelley and Mock, 1982; Carlson, 1967) with the maximum cold anomaly at $300 \mathrm{mb}$. If sufficiently shallow, a TUTT does not strongly perturb the low and mid-tropospheric "steering flow" of the tropical cyclone. The hurricane can therefore remain south of the subtropical ridge in the trade wind environment with relatively weak vertical shear. Intensification can therefore be both rapid and prolonged. Hurricane David 1979 exhibited this pattern as it 


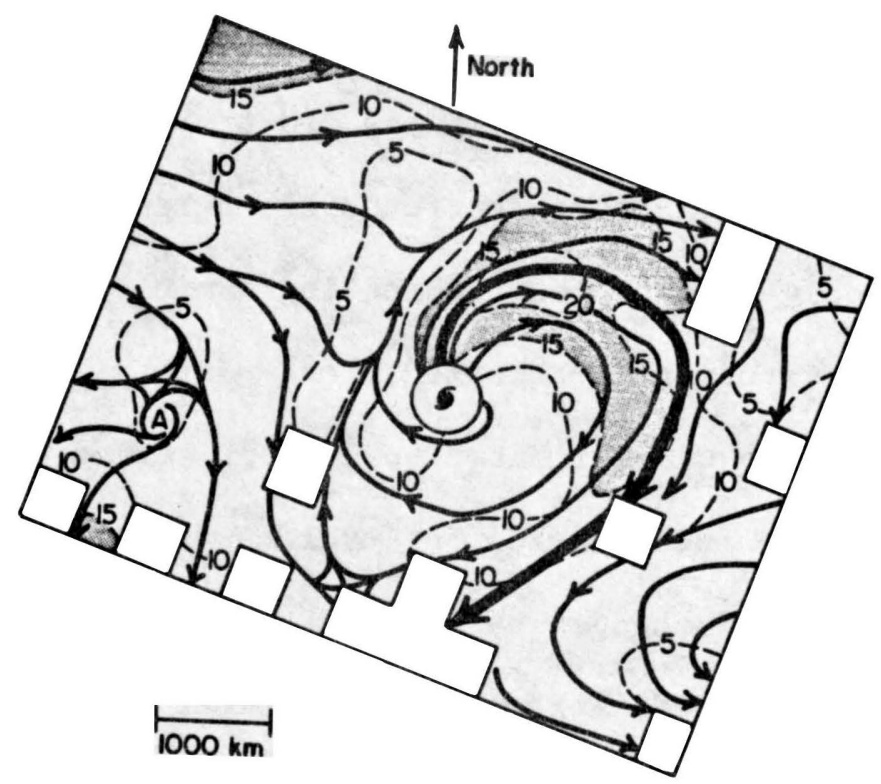

Fig. 3.3. Resultant upper-tropospheric winds $\left(m \mathrm{~s}^{-1}\right)$ for the outflow rotated (OROT) composite of hurricanes having a TUTT (tropical upper tropospheric trough) outflow pattern. The indicated "north" is defined as in Fig. 3.1.

traversed the western Atlantic and eastern Caribbean, attaining a minimum sea level pressure of $924 \mathrm{mb}$. In contrast, hurricanes with outflow ahead of a trough in the westerlies (subtropical jet or westerly trough pattern) would have their steering flow altered as well and would be drawn poleward and disrupted by increasing vertical shear after only a short period of intensification. Even the TUTT is not a favorable influence on tropical cyclones unless it is in the proper location. The strong westerly winds and associated vertical shear south of the semipermanent mid-Atlantic trough of summer are a frequent cause of non-development of disturbances and depressions. Clark (1983) and Case and Gerrish (1984) describe entire Atlantic hurricane seasons which were dominated by this phenomenon.

As hurricanes track (or form) in the more northerly or westerly latitudes of the tropical Atlantic, they are situated to the northwest 
of the mean TUTT location, and their outflow pattern, though still influenced by the upper trough, is of a different character. Rather than occupying a position to the northwest or north as is possible with a low-latitude hurricane, the TUTT now is evident as a more meridionally-oriented trough to the east or even southeast of the hurricane. Patterns of this type are the most prevalent in the Atlantic basin and are called Atlantic type $I$ and Atlantic type II, and similar configurations have been noted by Chen and Gray (1985) in the northwest Pacific, though less frequently.

Atlantic type I. Figure 3.4 shows the Cartesian grid composite resultant winds for Atlantic type I. This type is defined by the existence of an outflow streamilne which begins with a westerly component, curves anticyclonically, and recurves to an easterly component within $2500 \mathrm{~km}$ east of the cyclone center. A pattern is also classified as type I if the primary outflow streamline fails to recurve but a definable (section 2.5 ) outflow into the easterly stream equatorward of the hurricane exists. Subjectively, this pattern is characterized by extensive easterlies at low latitudes and strong northerly flow about $2000 \mathrm{~km}$ east of the hurricane, associated with an upper trough further to the east. These features are also evident in the composite.

Atlantic type II. Figure 3.5 shows the resul tant wind composite for the Atlantic type II, which is defined by an outflow streamline which drops below the latitude of the cyclone but falls to recurve within $2500 \mathrm{~km}$ east of the center. The type II is generally similar to the type I. Differences include an outflow streamline which goes off 


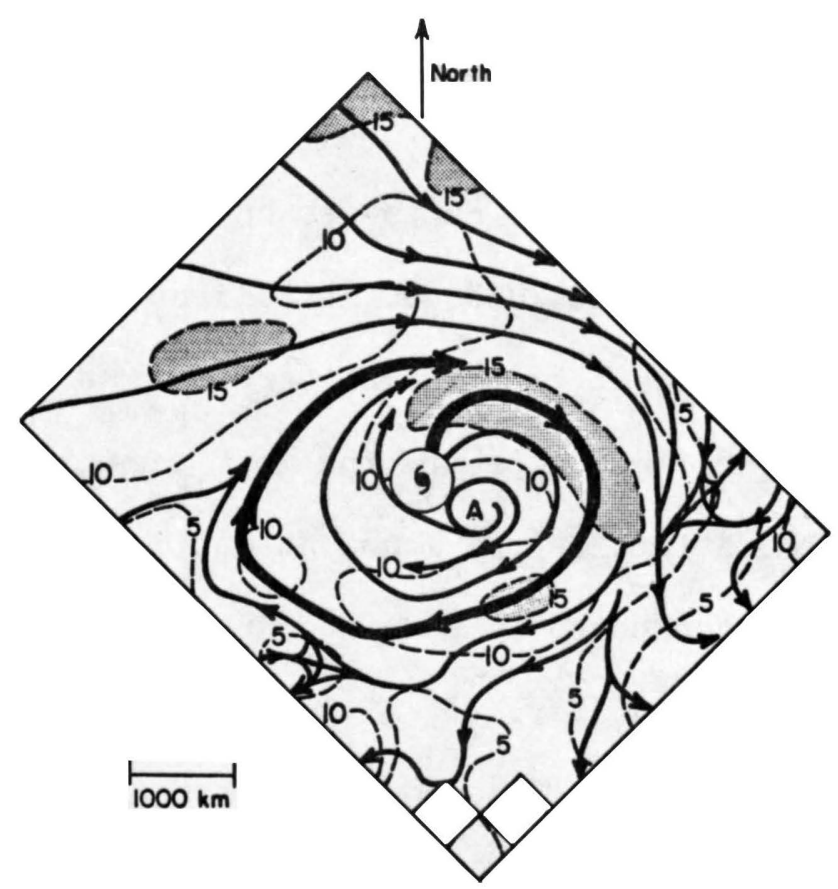

Fig. 3.4. Resul tant upper-tropospheric winds $\left(m \mathrm{~s}^{-1}\right)$ for the outflowrotated (OROT) composite of hurricanes having an Atlantic type I outflow pettern. The indicated "north" is defined as in Fig. 3.1.

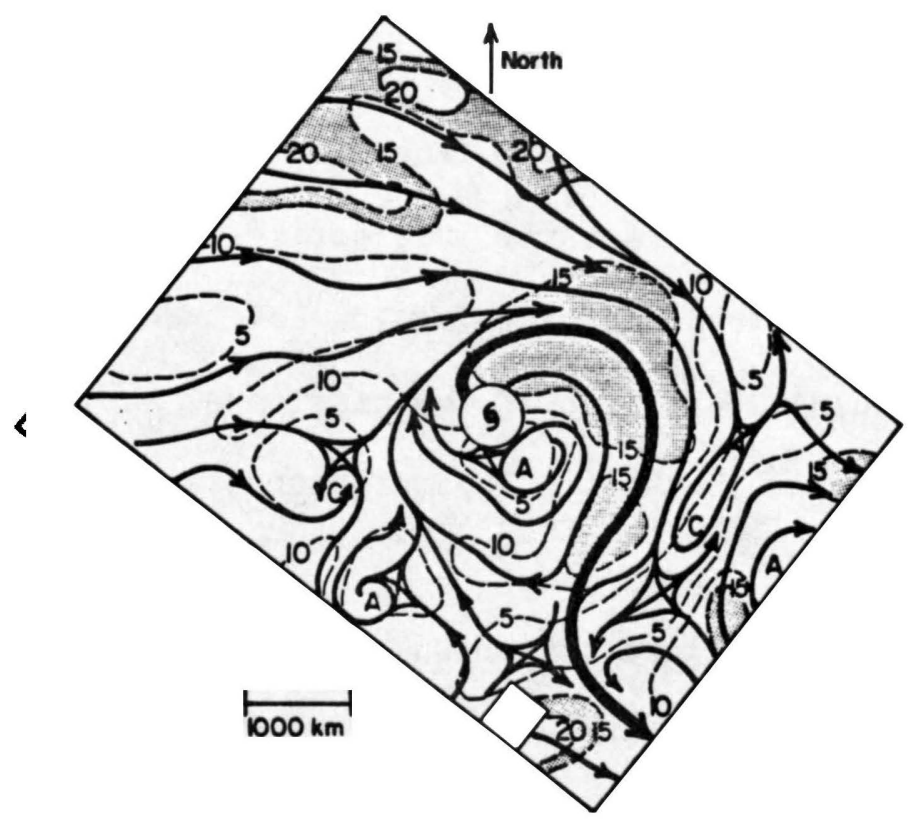

Fig. 3.5. Resultant upper-tropospheric winds $\left(\mathrm{m} \mathrm{s}^{-1}\right)$ for the outflowrotated (OROT) composite of hurricanes having an Atlantic type II outflow pattern. The indicated "north" is defined as in Fig. 3.1. 
the grid to the southeast, a more clearly defined trough to the east, and some semblance of an upper trough and cut of $f$ low to the westsouthwest. The type II generally occurs at higher latitudes than the type I, although individual hurricanes change between these two patterns relatively frequently. Hurricanes with these patterns fill and intensify with nearly equal frequency, and filling is often associated with increased upper-tropospheric shear. These sheared cases have been largely eliminated from Figs. 3.4-3.5 by compositing only those cases with $24 \mathrm{~h}$ maximum wind changes which are positive.

Westerly-trough Pattern. Figure 3.6 shows the mean resul tant wind for the westerly-trough pattern, associated with poleward-moving cyclones ahead of a major trough in the westerlies. Outflow is to the northeast, and the streamline is more than $1500 \mathrm{~km}$ north of the hurricane center when it crosses a line $1500 \mathrm{~km}$ each of the center. This pattern is similar to the subtropical jet pattern in that intensification is of ten rapid but short lived, and is terminated by upper-level shear which separates the hurricane's convection from the low level center, or by an extratropical transformation. The possibility of the latter makes this pattern a potentially dangerous one because of the possible simultaneous occurrence of a sudden course change and/or increase in intensity and/or strength as a large source of baroclinic energy release is suddenly tapped by an existing hurricane. Hurricanes Hazel 1954 (Palmen, 1958), and Eloise 1975 are notable examples of this explosive development. The tendency of typhoons to reach maximum intensity at or just prior to recurvature (Riehl, 1972) may be due to the development of this pattern type and the resulting enhancement of outflow mechanisms as a tropical cyclone recurves. 


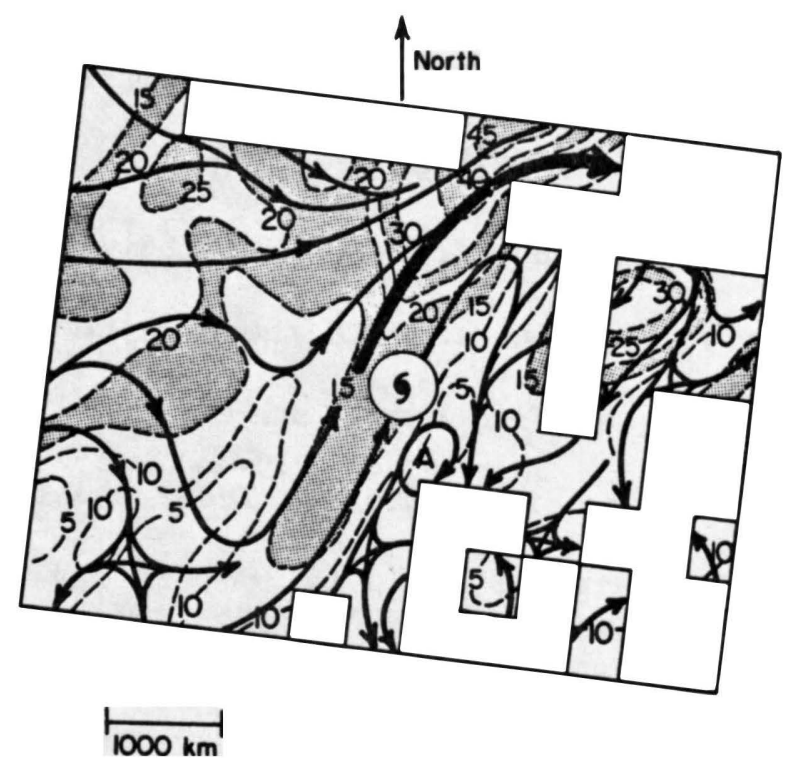

Fig. 3.6. Resultant upper-tropospheric winds $\left(m \mathrm{~s}^{-1}\right)$ for the outflowrotated (OROT) composite of hurricanes having a westerly trough outflow pattern. The indicated "north" is defined as in Fig. 3.1.

Assessment of the magnitude of the intensification to be expected according to the structure of the approaching trough remalns a difficult forecast problem. Perhaps the planned study of the extratropical "bomb," (Sanders and Gyakum, 1980) a midlatitude cyclone which Intensifles rapidly as oceanic energy is tapped, will shed additional Ilght on the behavior of cyclones in which both latent heating and baroclinic instability are 1mportant energy sources.

Easterly pattern. Only 5 positions (associated with Hurricane Allen in 1980) had their principal outflow to the west, and all were intensifying. Figure 3.7 shows the composite of this outflow type. Note the existence of an outflow jet to the northeast as well, such that this pattern resembles the double outflow channel patterns of chen and Gray (1985), which are especially conducive to intensification. Even in 


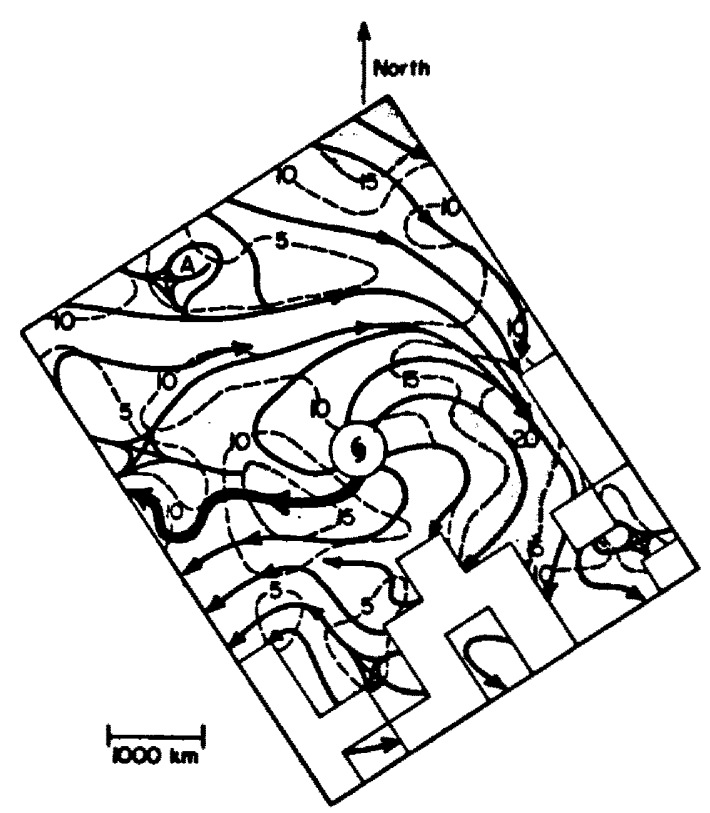

Fig. 3.7. Resul tant upper-tropospheric winds $\left(m \mathrm{~s}^{-1}\right.$ ) for the outflow rotated (OROT) composite of hurricanes having an easterly outflow pattern. The indicated "north" is defined as in Fig. 3.1.

individual cases, the easterly-flow outflow maximum is less

anticyclonically curved and less concentrated than is outflow into the westerlies. This pattern is apparently much more common in the Pacific, where the upper tropospheric equatorial easterlies are a climatological feature over the bulk of the tropical cyclone formation areas in mid season.

Gulf of Mexico pattern. The 7 positions exhibiting this pattern occurred with two Gulf of Mexico hurricanes, Anita 1977 and Allcia 1983 , both of which were intensifying. Caroline (1975), though before the period of record for this study, was also of this type (Rodgers and Gentry, 1983). The pattern (FIg. 3.8) is unique in that the large anticyclone is typicaliy located to the northwest of the hurricane 


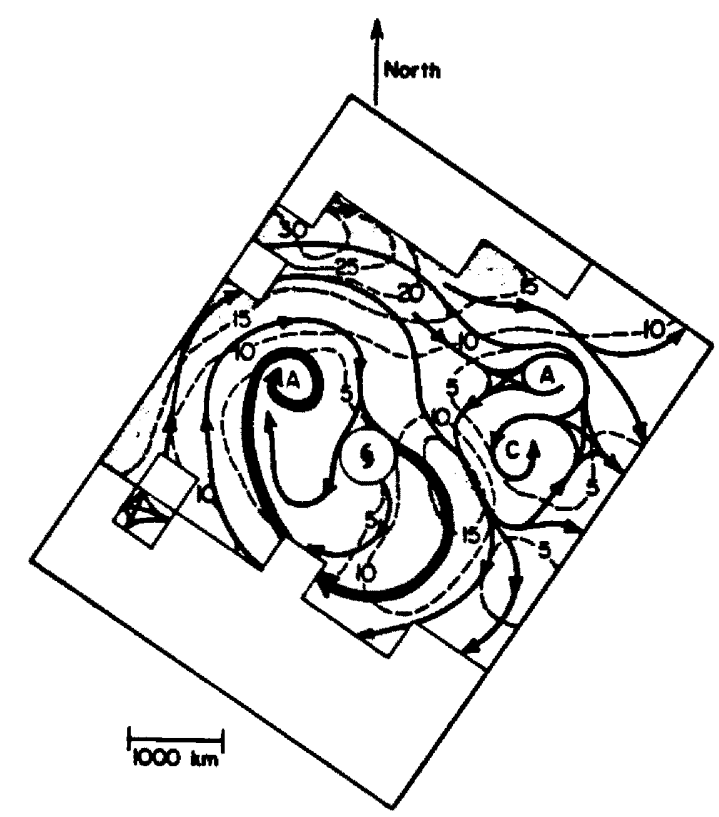

Fig. 3.8. Resul tant upper-tropospheric winds $\left(\mathrm{m} \mathrm{s}^{-1}\right)$ for the outflow rotated (OROT) composite of hurricanes having a "Gulf of Mexico" outflow pattern. The indicated "north" is defined as in Fig. 3.1 .

center, and is associated with the intense heating over subtropical North America in midsummer. The hurricane is located under the northerly flow between this large anticyclone and the western extremity of the TUTT. The pattern vaguely resembles an Atlantic type I or type II with the hurricane displaced 1500-2000 $\mathrm{km}$ to the east, under the northerly flow. A similar pattern is observed in the western Pacific (Chen and Gray 1985) in midsummer, when the synoptic situation in the outflow layer is dominated by the Tibetan anticyclone.

Discussion. Figures 3.1-3.8 represent the most comprehensive documentation of Atlantic hurricane outflow patterns to date. These patterns are summarized in Fig. 3.9. Because of the higher average latitude of Atlantic basin hurricanes (Gray, 1979) compared with the global mean, one might al so expect that the distribution of pattern types is different than that for the rest of the world. As previously 


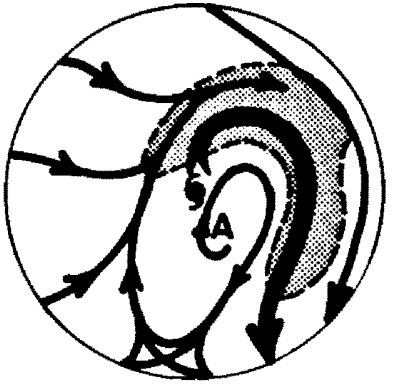

ATLANTIC TYPE ㅍ

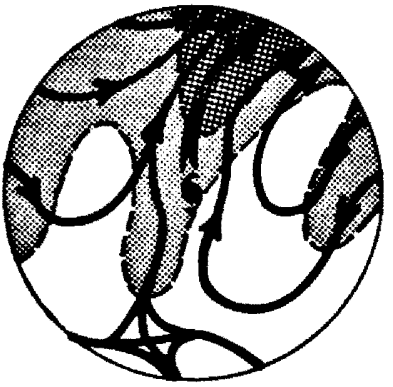

WESTERLY TROUGH

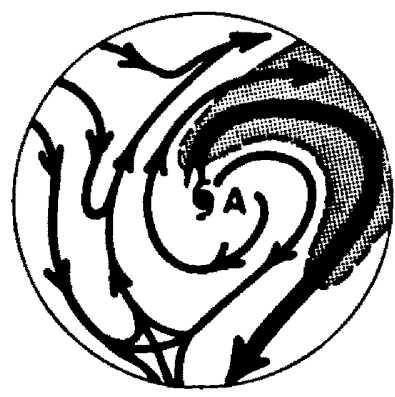

TUTT

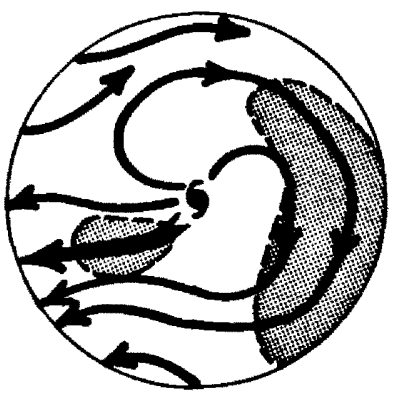

EASTERLY

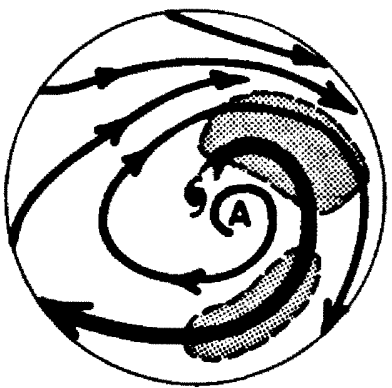

ATLANTIC TYPE I

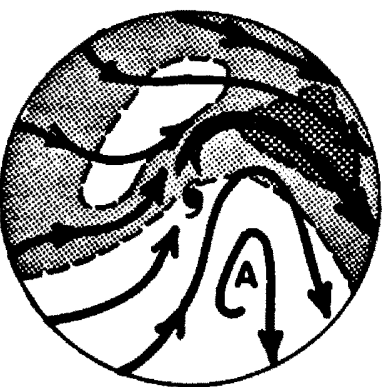

SUBTROPICAL JET

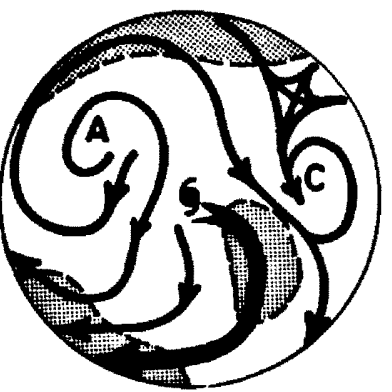

GULF OF MEXICO

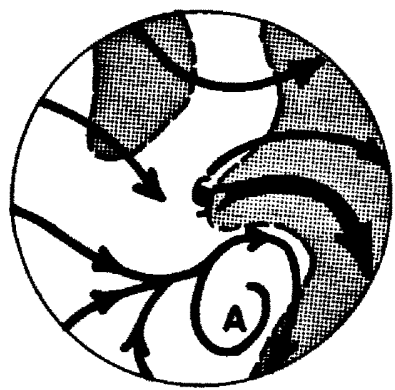

SUBTROPICAL ORIGIN

Fig. 3.9. Summary of Atlantic outflow pattern types shown separately in Figs. 3.1-3.8. Light shading and dark shading denote wind speeds in excess of $15 \mathrm{~m} \mathrm{~s}^{-1}$ and $30 \mathrm{~m} \mathrm{~s}^{-1}$ respectively, and the wide arrow shows the location of the principal outflow streamline. The circular domain of each analysis is $2500 \mathrm{~km}$ in radius. 
mentioned, Chen and Gray (1985) describe outflow patterns from a global perspective using the FGGE III-b analyses. Although their definition of an "outflow channel" is partially dependent on the satelitte cloud signature and is less rigid than that used in this paper, it can be inferred that outflow channnels to the equatorward side are more prevalent in the other basins of the Northern Hemisphere than in the Atlantic. This is perhaps due to the climatological lack of upper troposheric equatorial easterlies over the central and western Atlantic. The structure and dynamics of equatorial outflow merit a separate observational study because it differs in appearance from poleward outflow, yet is thought to have a similar effect on tropical cyclone behavior.

One of the motivations for this pattern classification was to supplement the outflow rotation composite methodology and resolve st1ll more of the variability in the hope that alfferences between intensifying and non-intensifying systems would be more apparent. Composites were thus made for Intensifying and filling cases for type I and type II, but the results were inconclusive. The filling systems had larger vertical shear over and near the hurricane center, but differences in outflow pattern were not readily apparent on the larger scales. Along the same line (though less likely, it was thought) was the possibility that certain patterns would be associated with intensification and others with filling. With the exception of the "Gulf of Mexico" and "Easterly" patterns, none of the pattern types appeared to be associated consistently with either filling or intensification. Comparison of composite characteristics for the various pattern types (and for other composites based on intensity 
tendency and not pattern) ylelded an interesting result. Much of the variability in intensity and intensity change was related to latitude and longltude. A similar result had been obtalned from a review by the author of the atmospheric variables selected by Dropco (1981) for Intensity change prediction; all selected predictors exhlbited a high correlation with a quadratic function of day number. These indications of variability of intensity change and intensity with location and time of year and without obvious regard to atmospheric flow conditions led to the re-evaluation of sea-surface temperature as a control on intensity and intensity change. Section 3.2 discusses past findings concerning this relationship and the development of a sea-surface temperature data base for this study, and section 3.3 describes the results of upper wind composites stratifled by Intensity change with sea-surface temperature taken into account.

\subsection{Sea-surface Temperature Influences on Hurricane Intensity}

The dependence of the hurricane upon the ocean has long been known. More than any other weather system the tropical cyclone is an oceanic disturbance. They develop over the oceans and, when borne over land by the prevalling winds, immediately begin to break up. The dependence has at various times been ascribed to reduced drag and Increased evaporation over the oceans. Riehl (1954) noted that as the alr flowed into the hurricane, the expansion and lowering of pressure was an isothermal, rather than adiabat1c, process. The heat was presumed to come from the sea surface. The partition of the flux between sensible and latent heat is still a subject of discussion, but the necessity of an oceanic energy source is agreed upon. 
The possibility of a dependence of intensity on sea surface temperature (SST) can be inferred in a variety of ways. Malkus and Riehl (1960) showed that transformation (by convective transports) of the mean tropical sounding to a molst-adiabatic sounding at the surface value of $\theta_{e}$ (equivalent potential temperature) resulted in a surface pressure of only $1000 \mathrm{mb}$, and only by an increase in $\theta_{e}$ by surface flux can a lower pressure be attained. Since $q_{s}$ (the mixing ratio at saturation) of alr goes up exponentially with temperature, the values of $\theta_{e}$ which are theoretically attainable by isothermal expansion and evaporation over an ocean surface rise sharply with increasing SST. Miller (1958), using a similar purely thermodynamic approach, estimated the character of eye soundings given low-level $\theta_{e}$ values, and presented a graph of minimum attainable pressure as a function of SST (Fig. 3.10). Early numerical simulations all indicated a dependence of intensity on SST, and SST sensitivity became a standard experiment for modelers (Ooyama, 1969; Sundqvist, 1970, 1972; Anthes and Chang, 1978). These numerical models lacked environmental influences and SST was of ten the factor controlling the peak intensity reached by the simulated hurricane. This result enjoyed a symblotic relationship with the CISKbased concept of the hurricane as an instab1lity phenomenon; once the instability was triggered, it would amplify until it reached the iimit imposed by the SST, subject only to the internal efficiency which affected the growth rate.

Observations of hurricane climatology also indicated an SST influence. Palmen (1948) and later Gray (1968) discussed and presented evidence of a low-temperature cut-off at $26.5^{\circ} \mathrm{C}$. Miller (1958) 


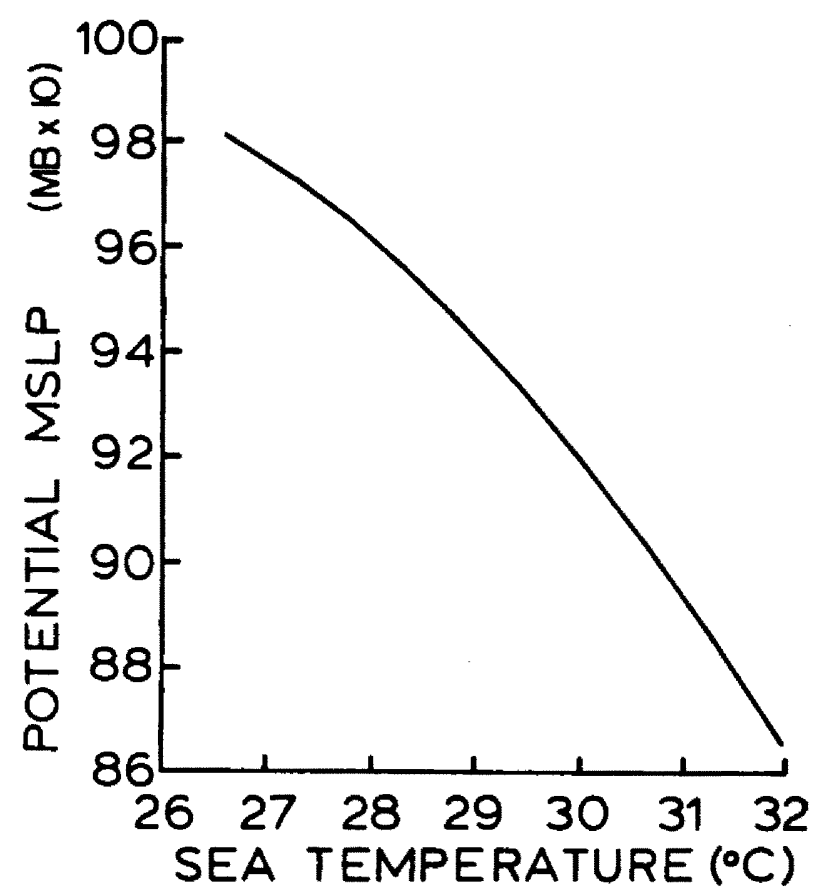

F1g. 3.10. Graph from Miller (1958) relating the theoretical minimum sea level pressure attainable by a tropical cyclone to the sea surface temperature. A discussion of Miller's work can be found in Chapter 4.

presented evidence that the minimum observed pressures in several intense hurricanes were close to the theoretical minimums as deduced from the SST but also stated that specific environmental conditions in the upper troposphere were necessary to reach the peak theoretical intensity. Despite Miller's comments, it apparently became commonplace In the late $1950^{\prime} s$ to relate intensity changes (al though not necessarily formation, for which an "Instability trigger" in the atmosphere was still sought) to changes in SST beneath the vortex, brought about by elther motion of the hurricane or upwelling induced by it. The idea of SST control of hurricane behavior reached its zenith in the work of Perlroth (1967) who attempted to relate motion, as well as intensity change, to the detalled SST structure. Perlroth presented several maps of hurricane tracks and SST and graphs of minimum pressure against SST (Fig. 3.11), and quoted a correlation of -0.7 between SST change and 


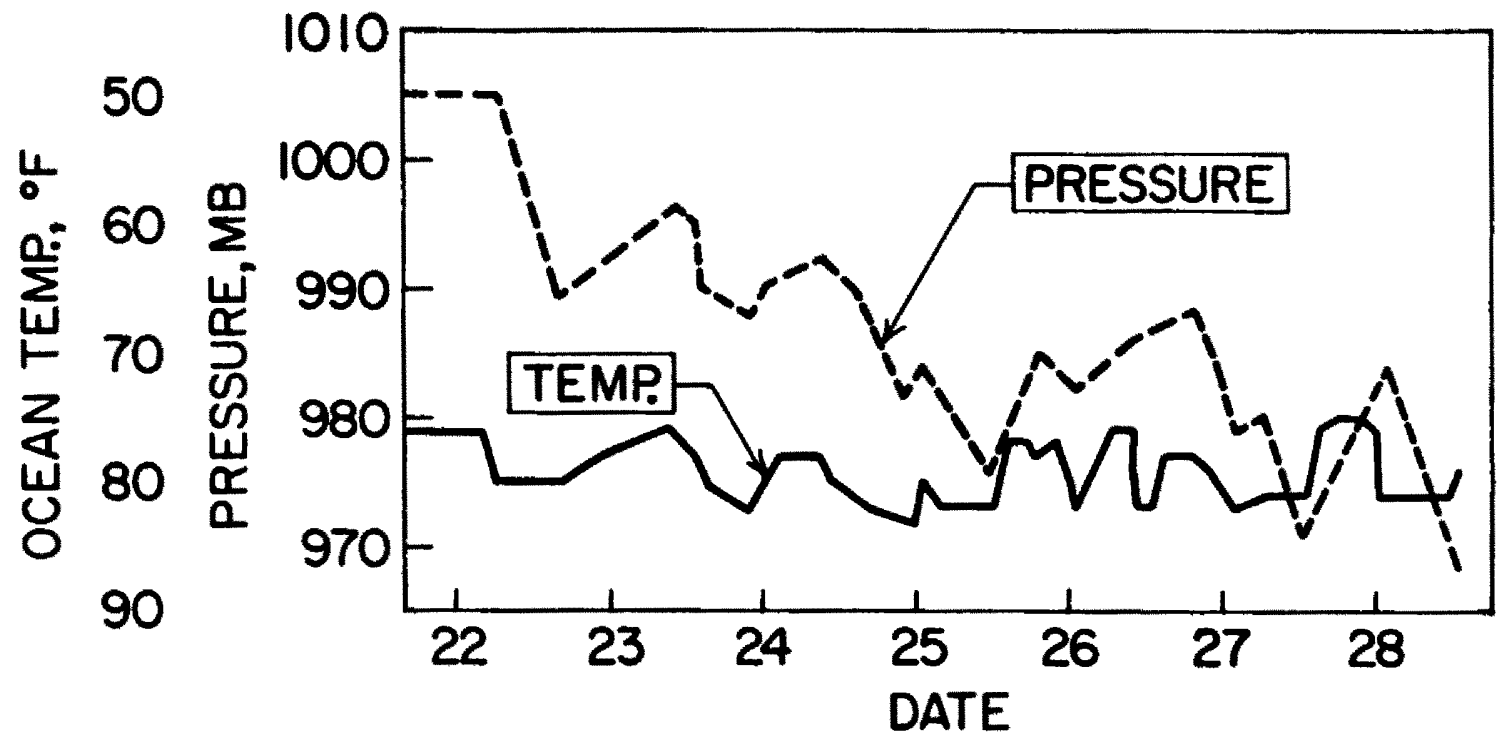

F \pm . 3.11. Relationship between minimum sea level pressure and sea surface temperature for Hurricane Ginny (1963). Figure from Perlroth (1967).

pressure changes over $12 \mathrm{~h}$ intervals. Though not commented on by Perlroth, Fig. 3.11 indicates that the direct relationship between Intensity and SST is much weaker. Note that SST of $80^{\circ} \mathrm{F}$ is associated with pressures ranging from 1005 to $970 \mathrm{mb}$ over the life of Ginny, indicating that the relationship only works for short time periods.

Criticism of the overly simplistic conclusions of Perlroth (1967) was soon forthcoming. Ramage (1974) stresses the case of a typhoon which intensified even though crossing the cold "wake" left in the ocean by a previous typhoon. It was concluded that the presence of a trough to the northwest was overriding the oceanic influence. Riehl (1979) presents the case of Hurricane Ella (1958) which, though extensively documented by research aircraft, received little attention In the literature because it falled to re-intensify over the warm Gulf of Mexico after weakening over Cuba. Riehl then reiterated the importance of enviromental flow conditions, specificaliy 
mass circulation through the vortex which was apparently lacking in the Ella case. Based on these findings and the criticism by forecasters of direct SST relationships with intensity change, SST was neglected altogether at the outset of this observational study. Its reintroduction in this research was prompted by the discouraging results of pattern classification as described at the end of section 3.1 .

The conceptual view adopted as a starting point was that of Miller (1958): SST imposes an upper bound, and environmental conditions determine the fraction of this maximum intensity actually reached. The proposed relationship is:

$$
\begin{gathered}
I \doteq E I_{S S T} \text { and } \\
\frac{d I}{d t} \doteq E \frac{d I_{S S T}}{d t}+I_{S S T} \frac{d E}{d t},
\end{gathered}
$$

where $I=$ current intensity, $E=$ efficiency, and $I_{S S T}=$ theoretical maximum intensity based on SST. Perlroth's conclusions regarding short-interval intensity changes in which SST variability dominates environmental effects can thus be viewed as

$$
\frac{d I}{d t_{\text {short }}}=E \frac{d I_{S S T}}{d t} .
$$

What we seek is the effect of the second term in Eq. 3.2, relating to the changes in efficiency, which we hypothesize are occurring more slowly, so that

$$
\frac{d I}{d t_{\text {long }}}=\left(I_{S S T}\right)_{\text {average }} \frac{d E}{d t}
$$


is more representative. Charts of mean monthly SST are considered to be an adequate representation of the SST influence for intensity and changes on time scales of a day or more. It is understood that shortInterval fluctuations in intensity may be associated with SST variability of $1^{\circ}-2^{\circ} \mathrm{C}$ on a time scale of $12 \mathrm{~h}$ (upwelling, etc.) or a space scale of $100 \mathrm{~km}$ (motion).

Monthly mean SST data were read from charts in Gorshkov (1979) for each hurricane position. During the first or last five days of each month, the value for the current month and the next nearest month were averaged. All SST values were then rounded to the nearest $0.5^{\circ} \mathrm{C}$. Once all hurricane positions have been assigned an SST value, it is then necessary to estimate the theoretical maximum intensity associated with a given value of SST. This was done by plotting the observed intensities of hurricanes for each $0.5^{\circ} \mathrm{C}$ class of SST (Fig. 3.12). The existence of an empirical "potential intensity" can be clearly seen, and it increases with increasing SST as expected. Either a straight line or a smooth curve can be fitted, and a function $V_{S S T}$ (the maximum wind possible for a given mean SST value) specified. For each position, the efficiency $E$ can then be estimated as $V_{\max } / V_{S S T}$, where $v_{\max }$ is the observed best-track maximum wind. Use of these efficiencies in place of actual wind speeds normalizes for the varying influence of SST and enables a somewhat clearer look at the environmental flow differences between intensifying and non-intensifying hurricanes.

\subsection{Composites of Intensity and Intensity Change Normalized According to Sea Surface Temperature \\ After each position was classified according to sea surface temperature and efficiency, the positions were stratified for compositing purposes. Stratification was first made for sea temperature}




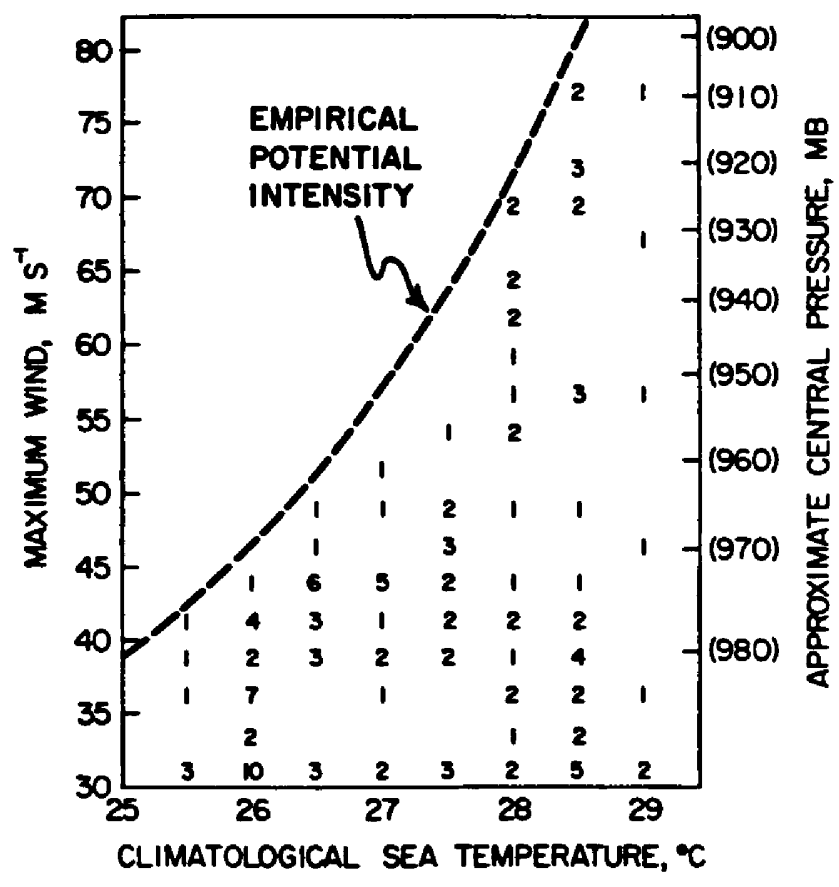

Fig. 3.12. Empirical relationship between sea surface temperature and best-track maximum wind for the sample of hurricanes used in upper tropospheric wind compositing. The dashed line is an empirical upper bound on intensity as a function of SST.

(warm or cold water), then for intensity (expressed as an efficiency or normalized wind speed) and lastly for intensity change (change in best track wind speed over the $24 \mathrm{~h}$ period centered on the position time). Efficiency and intensity change stratificaticn criteria were chosen so as to divide the sample into nearly equal halves. In initial composite analysis with these relatively small samples, it was found that inclusion of even one position with an atypical (for the sample) outflow pattern or rotation angle tended to distort the flow, particularly at the larger radi1, by the ef fect discussed in section 2.5 . Such distortions make comparisons between sets difficult, so the samples were further screened and the cases with extreme rotation angles were 
removed, such that all rotation angles in the final sample for each fell within a $120^{\circ}$ range.

The fine stratifications and the elimination of "hidden" sources of intensity change variability carries with it a price; the sample sizes are small and often draw all of the positions from 2 or 3 cyclones. The results will therefore be discussed in a general, largely non-quantitative way. What is most striking is the lack of "obvious" differences. Perhaps this explains the historically frustrating nature of this topic of environmental effects on intensity change. The differences are largely "second-order" and are easily obscured by other sources of variance, most notably current intensity and sea surface temperature (which is also related to latitude and season and thus to the mean planetary scale flow, which may or may not be related to the hurricane's behavior). One important factor which has been neglected is strength (Weatherford, 1985) which may be related to the bulk properties of the outflow (total mass circulation and total momentum flux) as well.

The first palr of composites to be considered are the low efficlency $\left(V_{\max } / V_{S S T} \leq .65\right)$ warm water $\left(S S T \geq 28^{\circ} \mathrm{C}\right)$ results. Figure 3.13 is the intensifying composite $\left(24 \mathrm{~h}\right.$ wind increase of $7.5 \mathrm{~m} \mathrm{~s}^{-1}$ or more) and Fig. 3.14 is the non-intensify ing composite (wind inorease of less than $7.5 \mathrm{~m} \mathrm{~s}^{-1}$ in $24 \mathrm{~h}$ ). The average intensities are $41 \mathrm{~m} \mathrm{~s}^{-1}$ and $37 \mathrm{~m} \mathrm{~s}^{-1}$ and $24 \mathrm{~h}$ maximum wind changes changes $14 \mathrm{~m} \mathrm{~s}^{-1}$ and $1 \mathrm{~m} \mathrm{~s}^{-1}$, respectively. The two systems are qualitatively similar; both show outflow to the northeast of the cyclone center, a closed anticyclone of around $1000 \mathrm{~km}$ radius, and an envirormental flow of westerlies to the poleward side. Intensifying systems show: 1) 


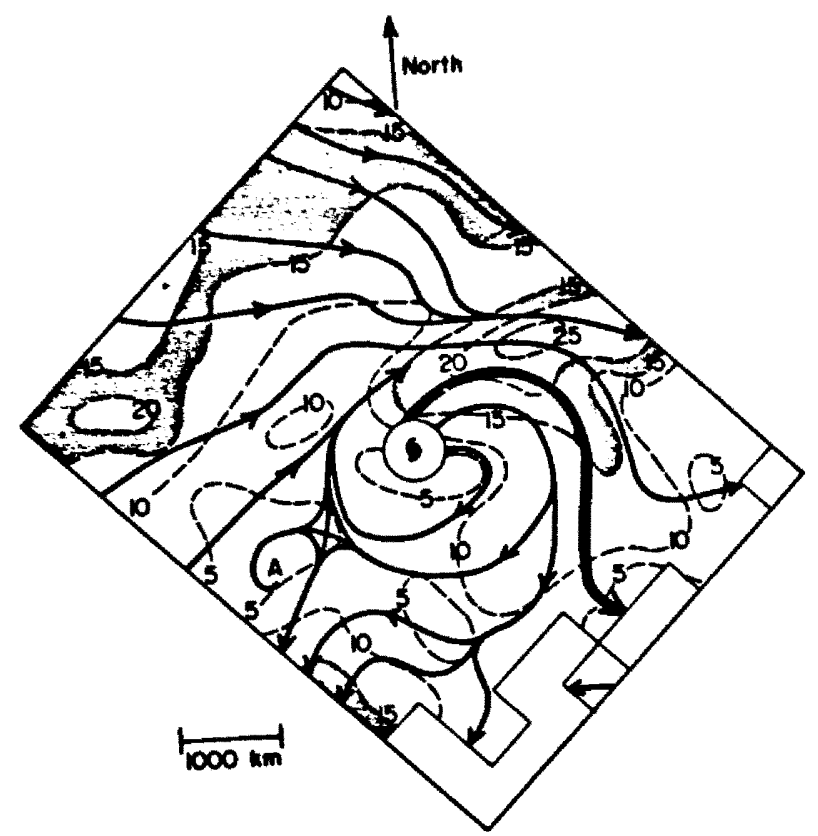

FIg. 3.13. Resultant upper-tropospheric winds ( $\mathrm{s}^{-1}$ ) for the outflow rotated (OROT) composite of warm water (SST $\geq 28^{\circ} \mathrm{C}$ ), low efficiency $\left(V_{\max } / V_{S S T}<.65\right)$, intensifying $\left(\Delta V_{\max } / 24 \mathrm{~h} \geq 7.5\right.$ $\mathrm{m} \mathrm{s}^{-1}$ ) hurricanes. The indicated "north" is defined as in FIg. 3.1.

stronger flow speeds at distances of $1500 \mathrm{~km}$ and beyond to the northeast, 2) weaker northerly flow along the east flank of the anticyclone, 3) a more prominent trough far to the east $(2000 \mathrm{~km}$ or more), 4) weaker southwesterly flow to the southwest, and 5) stronger westerlies far to the west with a tendency towards ridging to the northwest. Comparison of Figs. 3.13 and 3.14 reveals that the flow speed in the outflow streamline is weaker for the intensifying case both at the $1000 \mathrm{~km}$ radius used to define the rotation angle and further downind along the east flank of the anticyclone. This is opposite of what might be expected. The "enhancement" of the intensifying cyclone's outflow consists of item 1) above; compare the shape of the 20 $\mathrm{m} \mathrm{s}^{-1}$ isotach northeast of the center in Figs. 3.13 and 3.14 . 


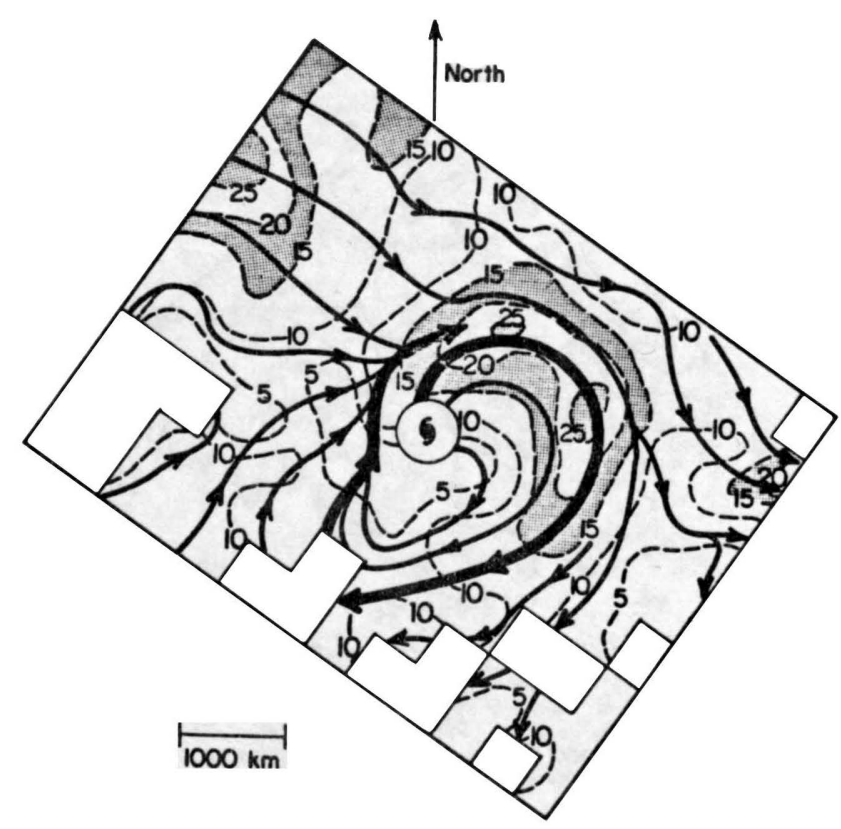

F1g. 3.14. As Fig. 3.13 except for non-intensifying $\left(\Delta V_{\max } / 24 \mathrm{~h}<7.5 \mathrm{~m}\right.$ $\mathrm{s}^{-1}$ ) hurricanes.

The high efficiency $\left.\left(V_{\max } / V_{S S T}\right) .65\right)$ warm water composites (Figs. 3.15 and 3.16) show fewer obvious differences than the low-efficiency cases. Both are relatively "heal thy" systems (maximum winds of $66 \mathrm{~m}$ $\mathrm{s}^{-1}$ for the intensifying set and $67 \mathrm{~m} \mathrm{~s}^{-1}$ for the non-intensifying set), and the intensity change difference $\left(+15 \mathrm{~m} \mathrm{~s}^{-1}\right.$ and $\left.-5 \mathrm{~m} \mathrm{~s}^{-1}\right)$ is large. The greatest flow difference lies to the northwest, with ridging in the intensifying case and troughing in the non-intensifying case. The lowefficlency palr (Figs. 3.13 and 3.14 ) also show this difference but to a lesser extent. Pattern "constriction" (in which the outflow streamline doubles back on itself as in Fig. 3.14) is not noticeable in either of the high-efficiency sets, although the outflow streamline is $500-750 \mathrm{~km}$ closer to the center during its run down the eastern flank of the outflow anticyclone in the non-intensifying case. Part of the difficulty with this comparison may be the specification of "intensification" or "non-intensification" based on a $24 \mathrm{~h}$ centered 


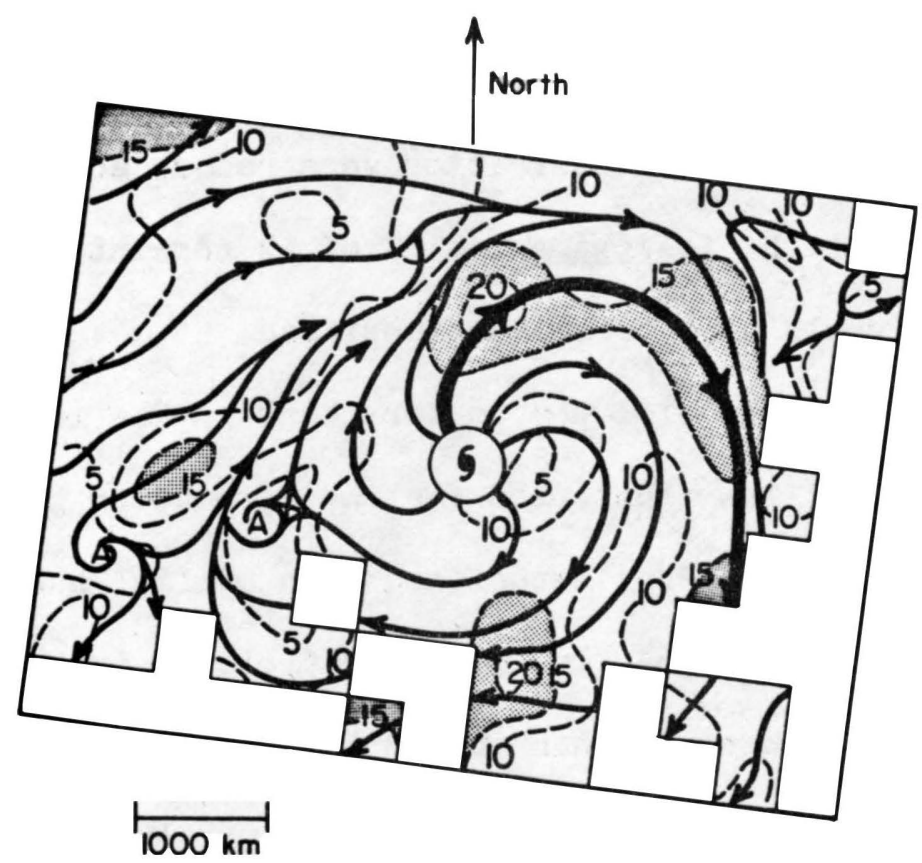

Fig. 3.15. Resul tant upper-tropospheric winds $\left(\mathrm{m} \mathrm{s}^{-1}\right)$ for the outflowrotated (OROT) composite of warm water (SST $>28^{\circ} \mathrm{C}$ ), high efficiency $\left(V_{\max } / V_{S S T}>.65\right)$, intensifying $\left(\Delta V_{\max } / 24 \mathrm{~h} \geq 7.5\right.$ $m \mathrm{~s}^{-1}$ ) hurricanes. The indicated "north" is as defined in Fig. 3.1.

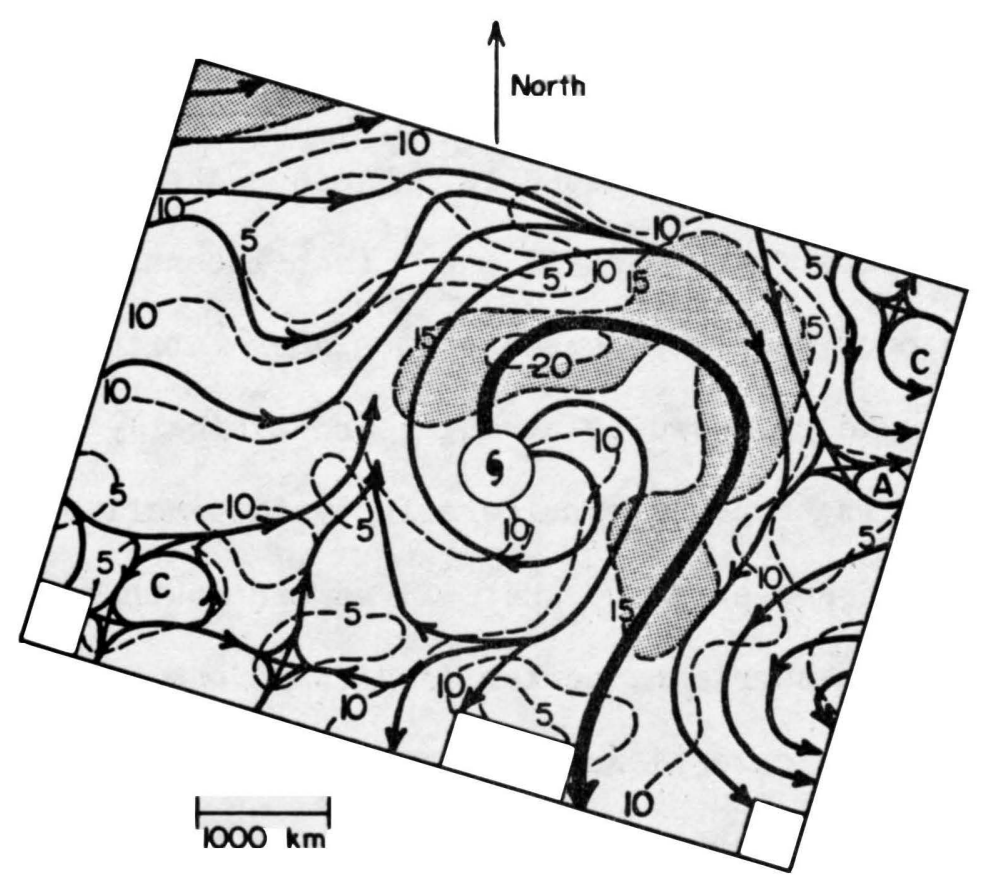

Fig. 3.16. As Fig. 3.17 except for non-intensifying $\left(\Delta V_{\max } / 24 \mathrm{~h}<7.5\right.$ m $\mathrm{s}^{-1}$ ) hurricanes. 
difference. At the end of this section, evidence that the outflow character is a leading indicator of intensity change will be discussed. If the intensity change classification were redone according to maximum wind tendency over the following $24 \mathrm{~h}, 3$ of 12 non-intensifiers and 4 of 9 intensifiers would have to be re-classified.

Cool water systems will now be considered. The intensity threshold of .74 is different than that used for the warm water group because $V_{S S T}$ is lower, and the efficiency associated with minimal hurricane intensity is higher, over cool water. The median efflciency is thus higher for the cool water set when only hurricanes are included. This does not imply that cool water systems are more efficient in an energetic sense, but only that more of them are near the observed upper limit of intensity. This could be due to the fact that the lowest possible efficiency (minimal hurricane) is higher, or to the presence in cool water areas of weakening hurricanes from lower latitudes.

The cool water $\left(S S T 26.5-27.5^{\circ} \mathrm{C}\right)$ low efficiency patr $\left(V_{\max } / V_{S S T} \leq\right.$ .74) is shown in Figs. 3.17 and 3.18. These systems have maximum winds of $40 \mathrm{~m} \mathrm{~s}^{-1}$ (Intensifying) and $38 \mathrm{~m} \mathrm{~s}^{-1}$ (non-intensifying). As would be expected, the cool water systems are in higher latitudes and the westerly stream to poleward is stronger and typically more extensive. This comparison is hampered considerably by the small sample size, but differences which are apparent are: 1) weaker southwesterlies over and to the west of the hurricane center (and therefore less vertical shear) In the intensifying case 2) anticyclone centered over the hurricane in the intensifylng case and displaced $1000 \mathrm{~km}$ to the east southeast in the filling case, 3) greater lateral extent of the $20 \mathrm{~m} \mathrm{~s}^{-1}$ isotach 


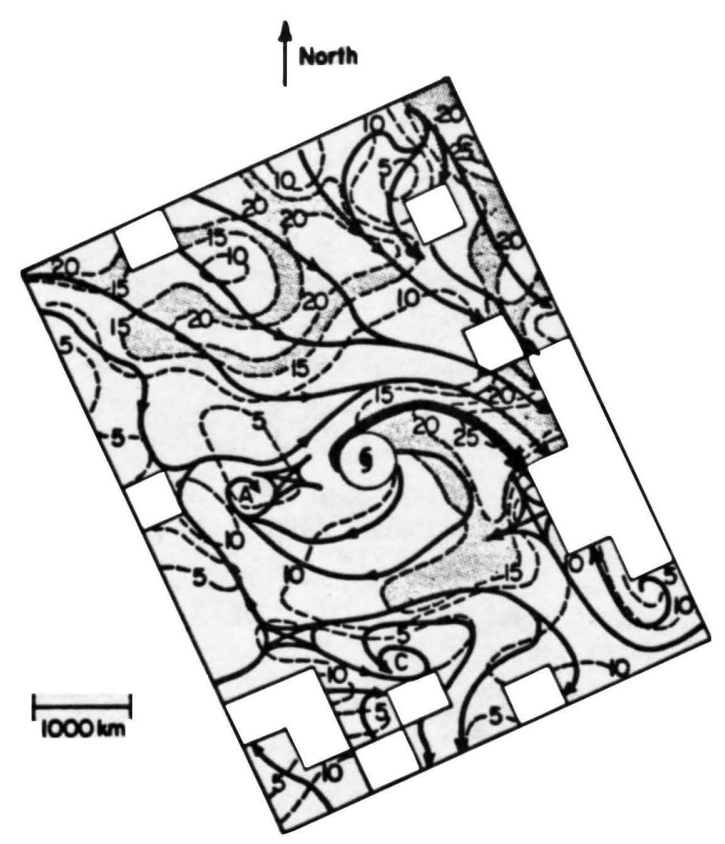

Fig. 3.17. Resultant upper-tropospheric winds $\left(\mathrm{m} \mathrm{s}^{-1}\right)$ for the outflow rotated (OROT) composite of cool water $\left(26.5^{\circ} \mathrm{C} \leq \mathrm{SST} \leq\right.$ $\left.27.5^{\circ} \mathrm{C}\right)$, low efficiency $\left(V_{\max } / V_{S S T} \leq .74\right)$, intensifying $\left(\Delta V_{\max } / 24 \mathrm{~h} \geq 5 \mathrm{~m} \mathrm{~s}^{-1}\right)$ hurricanes. The indicated "nor th" is as defined in Fig. 3.1 .
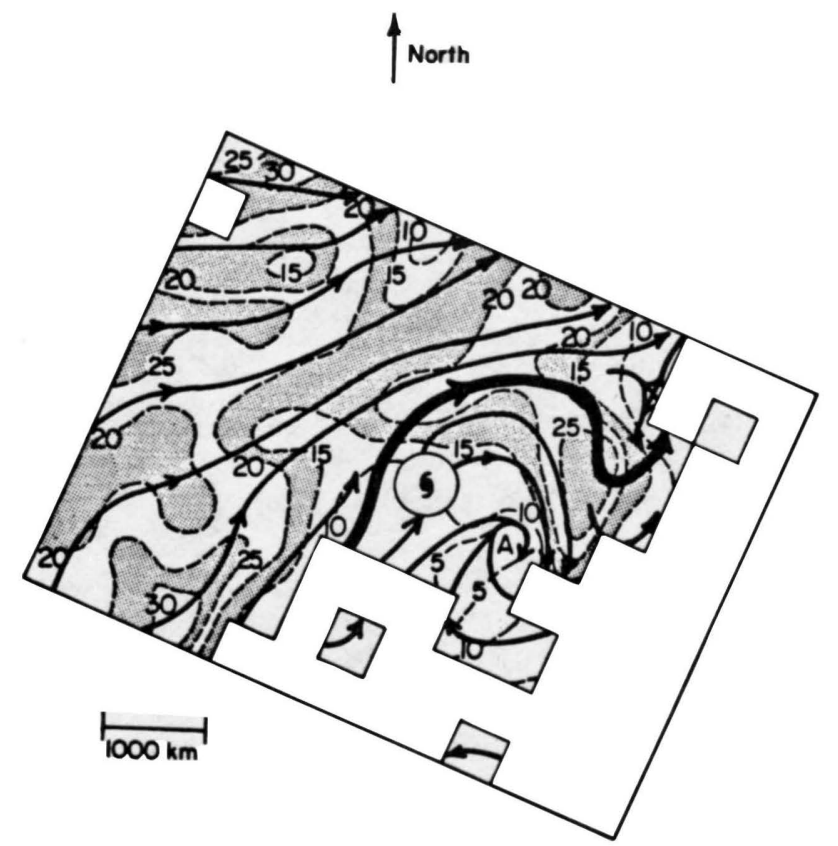

Fig. 3.18. As Fig. 3.17 except for non-intensifying $\left(\Delta V_{\max } / 24 \mathrm{~h}<5 \mathrm{~m}\right.$ $\mathrm{s}^{-1}$ ) hurricanes. 
assoclated with the outflow jet, 4) lack of troughing upstream, and 5) lack of pattern constriction (outflow streamlines do not double back) in the intensifying case.

The cool water high efficiency pair are shown in Fig. 3.19 (intensifying $2.5 \mathrm{~m} \mathrm{~s}^{-1}$ or more in $24 \mathrm{hr}$ ) and Figs. 3.20 (intensifying less than $2.5 \mathrm{~m} \mathrm{~s}^{-1}$ or filling in $24 \mathrm{hr}$ ). Agatn, the non-intensifying case appears vertically sheared, its outflow anticyclone relatively isolated, outflow speed maximum of limited nor theastward extent, and flow upstream dominated by southwesterlies. The intensifying case has low vertical shear, a trough to the west, an anticyclone upstream, and a radially extensive speed maximum associated with the outflow jet. Unlike previous cases, the intensifying system also has stronger outflow speeds within and at $1000 \mathrm{~km}$ radius.

\subsection{Temporal Changes of Outflow Character During Intensification}

Based upon the results described above, it is asserted that systematic, though subtle, differences in outflow pattern exist between intensifying and non-intensifying hurricanes. It can still however be argued that the differences are: a) coincidental differences between pairs of cyclones which were intensifying or filling for some other reason and are not applicable to the temporal progression of outflow development in an individual case, or b) passive differences arising subsequent to a change in the hurricane's behavior.

From the total sample described in section 2.2 , hurricanes were sought which showed a continuous, monotonic increase in efficiency $\left(V_{m} / V_{S S T}\right)$ for at least $24 \mathrm{~h}$ ( 3 positions), followed by a period in which the efficiency did not increase but the winds remained above hurricane force. Six candidates were found: David 1979, Frederic 1979, Allen 


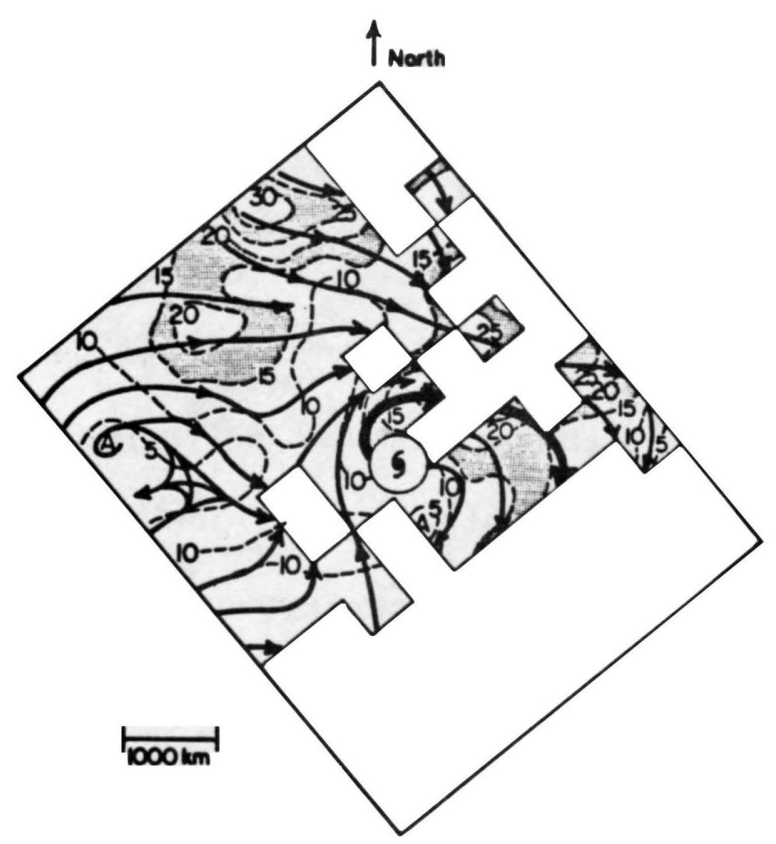

Fig. 3.19. Resultant upper-tropospheric winds $\left(m \mathrm{~s}^{-1}\right)$ for the outflow rotated (OROT) composite of cool water $\left(26.5^{\circ} \mathrm{C} \leq \mathrm{SST} \leq\right.$ $\left.27.5^{\circ} \mathrm{C}\right)$, high efficiency $\left(\left(\Delta V_{\max } / V_{S S T}\right) .74\right)$, intensifying $\left(\Delta V_{\max } / 24 \mathrm{~h} 22.5 \mathrm{~m} \mathrm{~s}^{-1}\right)$ hurricanes. The indicated "north" is as defined in Fig. 3.1.

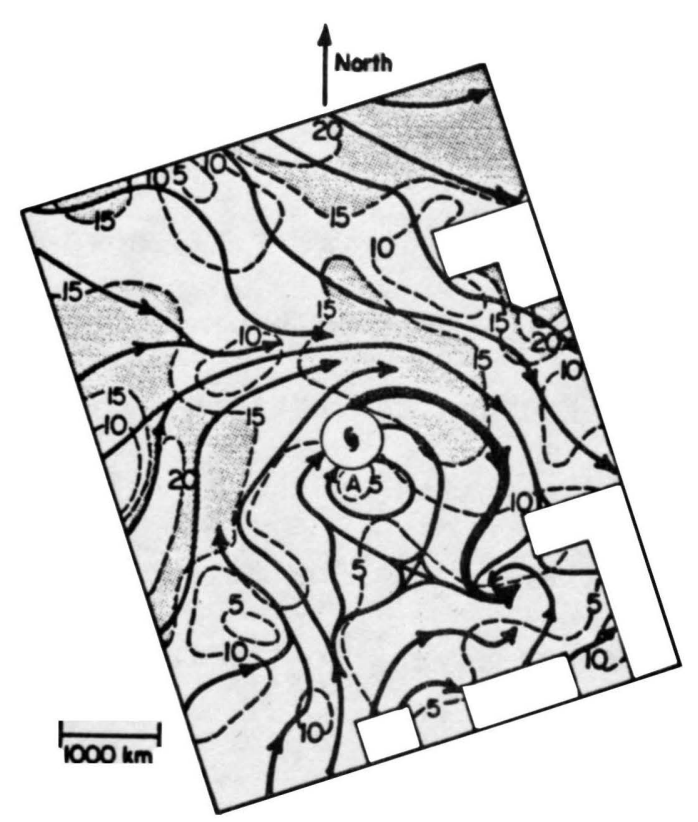

F18. 3.20. As FIg. 3.21 except for non-intensifying $\left(\Delta V_{\max } / 24 \mathrm{~h}<2.5 \mathrm{~m}\right.$ $\left.\mathrm{s}^{-1}\right)$ hurricanes. 
1980 (two occurrences, designated Allen-1 and Allen-2). Frances 1980 , Floyd 1981, and Harvey 1981. The positions for each hurricane were then assigned to one of three groups. The first two positions of a $36 \mathrm{~h}$ deepening period (first position of a $24 \mathrm{~h}$ period for David and Allen-2 were assigned to "early intensifying", the following two positions (the second of which is the position at which peak efficiency for that hurricane is attained) to "late intensifying" and the following one or two positions to "filling". Upper tropospheric composites were then made for each group to illustrate the temporal changes in outflow layer structure as a hurricane intensifies, peaks, and begins to fill. The resultant winds are shown in Fig. 3.21 (early), Fig. 3.22 (late), and Fig. 3.23 (filling).

The mean radial winds (not shown) do not change substantially or consistently. Tangential winds become progressively more negative beyond 6 degrees radius between the early and late stages, with a decrease from $-9.4 \mathrm{~m} \mathrm{~s}^{-1}$ to $-12.6 \mathrm{~m} \mathrm{~s}^{-1}$ in the 9-11 0 latitude radius belt. Comparing Fig. 3.21 and Fig. 3.22 shows this increase in anticyclonic flow, particularly to the southeast. The outflow also becomes more constricted and the pattern more symmetric. Also apparent is an anticycionic progression of the outward extension of the wind maximum associated with the outflow at a speed of motion of about $5 \mathrm{~m} \mathrm{~s}^{-1}$.

From the late intensifying to the flling stage the tangential winds continue to become more anticyclonic beyond $10^{\circ}$ radius (decrease from $-8.5 \mathrm{~m} \mathrm{~s}^{-1}$ to $-11.8 \mathrm{~m} \mathrm{~s}^{-1}$ in the $13-15^{\circ}$ radius belt) and the filling system's outflow (Fig. 3.23) is clearly constricted. A data sparse area (density of 5 observations or less per grid point) obscures 


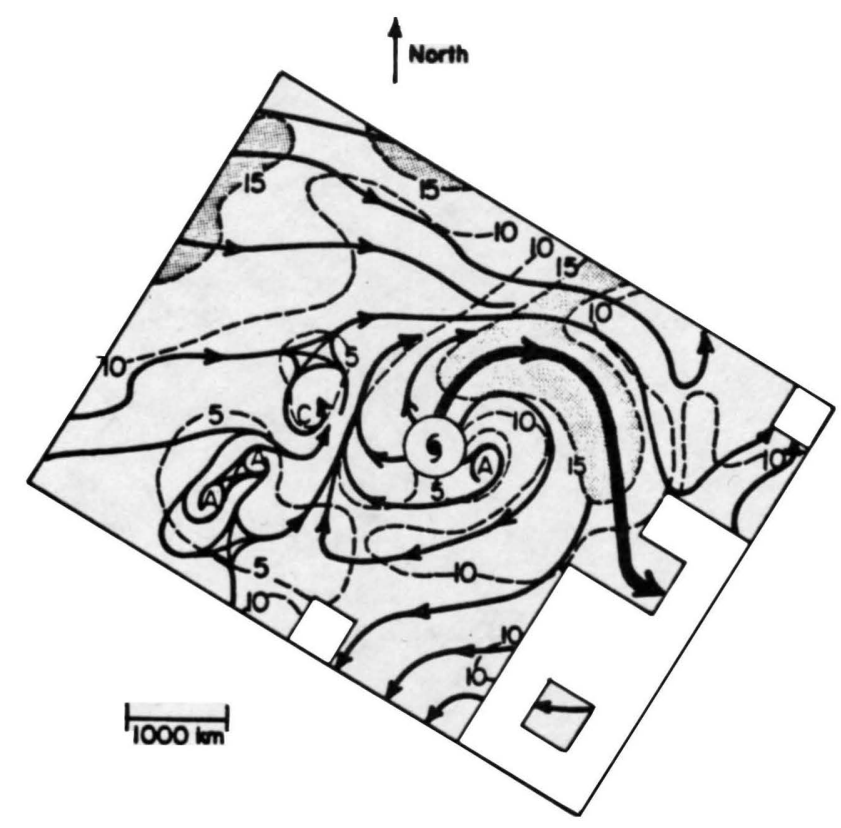

F1g. 3.21. Resul tant upper-tropospheric winds $\left(n \mathrm{~s}^{-1}\right)$ for the outflowrotated (OROT) composite early Intensifying hurricanes. The average intensity is $48 \mathrm{~m} \mathrm{~s}^{-1}$ and intensity increase is $16 \mathrm{~m}$ $\mathrm{s}^{-1}$ in $24 \mathrm{~h}$. The indicated "north" is as defined in Fig. 3.1.

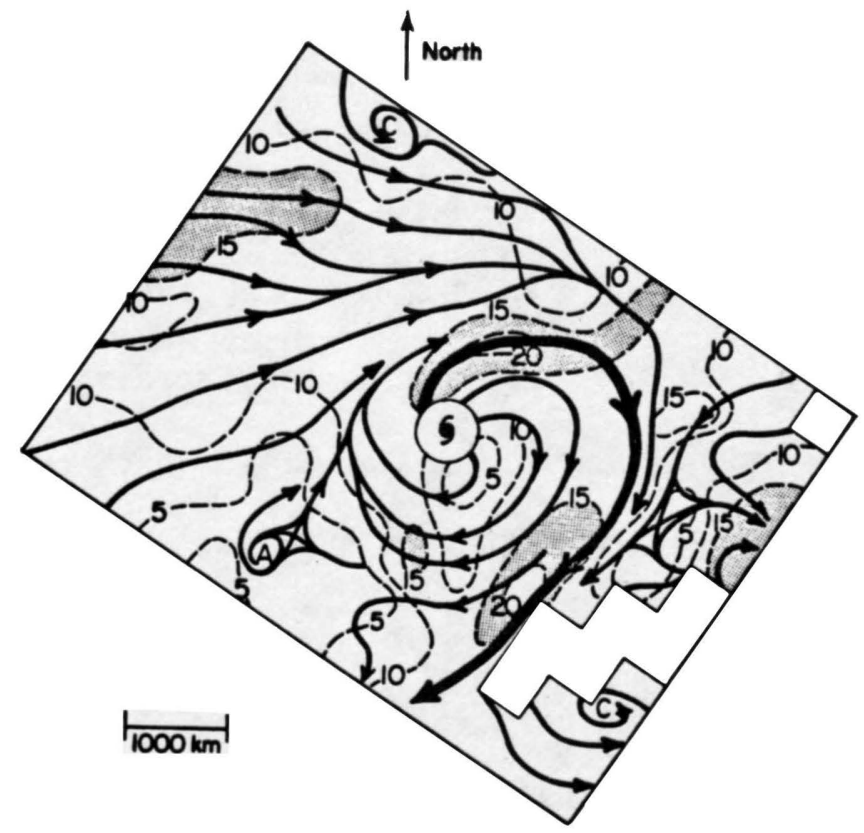

Fig. 3.22. As Fig. 3.21 except for late intensifying hurricanes. The average intensity is $62 \mathrm{~m} \mathrm{~s}^{-1}$ and intensity increase is $6 \mathrm{~m}$ $\mathrm{s}^{-1}$ in $24 \mathrm{~h}$. 


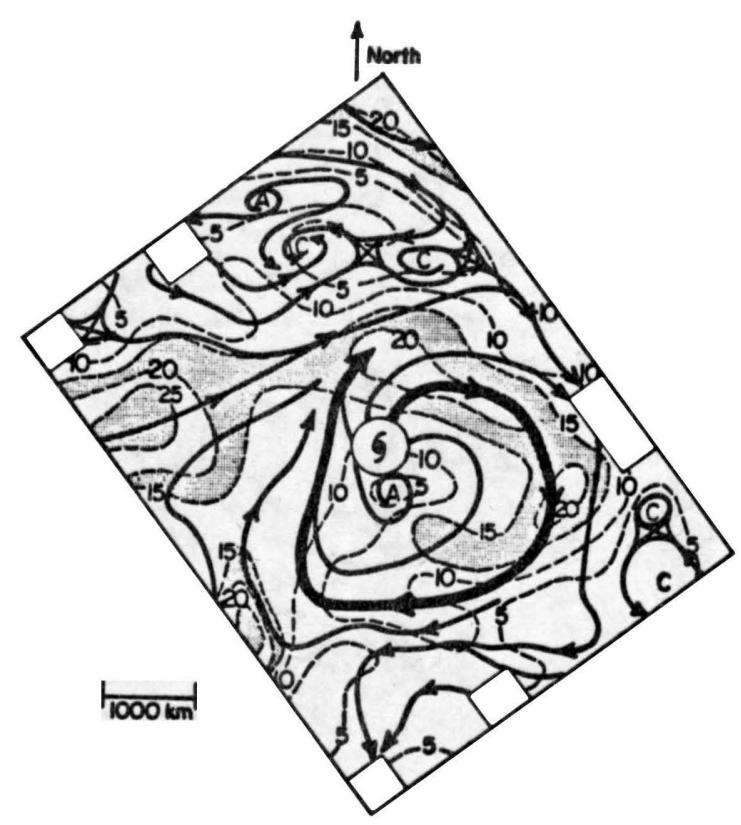

Fig. 3.23. As Fig. 3.21 except for steady/filling hurricanes. The average intensity is $55 \mathrm{~m} \mathrm{~s}^{-1}$, and decreases an average of $10 \mathrm{~m} \mathrm{~s}^{-1}$ in $24 \mathrm{~h}$.

the location of the radial elongation of the outflow maximum, but a continued anticyclonic motion of this region is weakly indicated.

A tendency towards the "non-intensifying" flow characteristics described in section 3.3 (more negative tangential flow at outer radii and a more constricted outflow streamline pattern seems to occur as a hurricane "peaks", with the tendency already apparent even while intensification is still occurring. Changes in outflow character may therefore furnish a "leading indicator" for at least the cessation of intensification.

\subsection{Summary of Observational Results}

Comparisons of outflow layer composites made for intensifying and non-intensifying hurricanes have revealed differences which are summarized in Fig. 3.24. This is a collection of differences, and no 


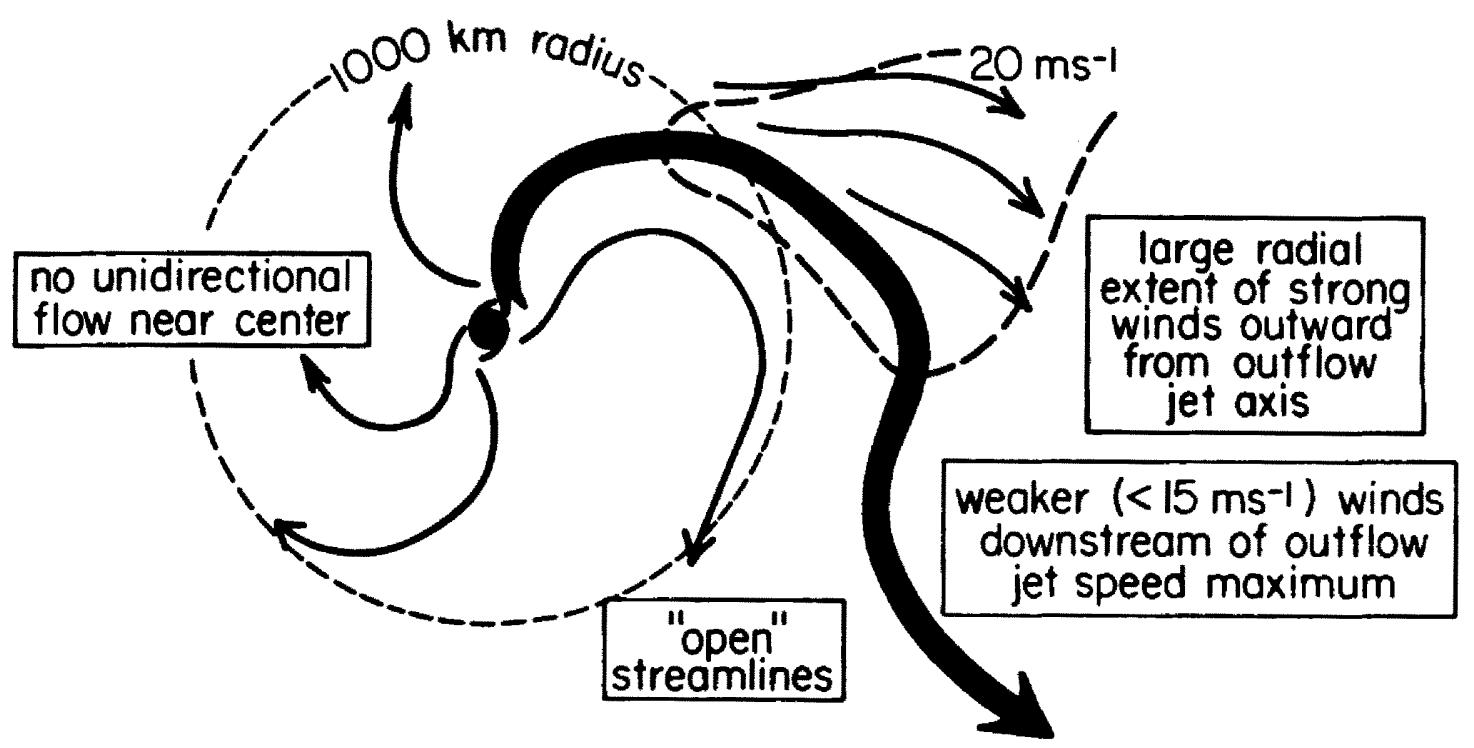

INTENSIFYING

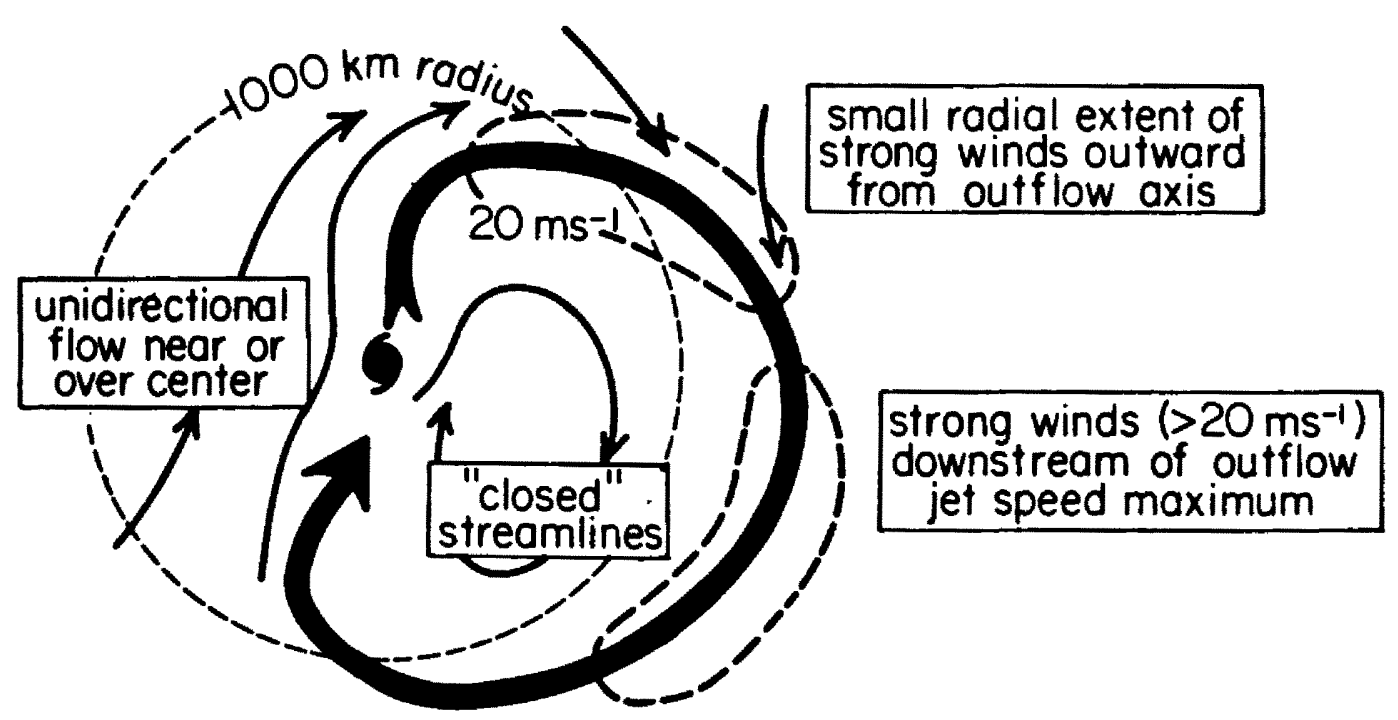

NON-INTENSIFYING

F18. 3.24. Summary of outflow differences between intensifying (top) and non-intensifying (bottom) hurricanes. 
one composite pair shows all of them. In particular, unidirectional flow over the center appears to be a feature of filling over cooler water (at higher latitudes) while pattern constriction is more of a warm water phenomenon. The greater radial extent of strong winds outward from the outflow channel appears in both warm and cold water differences, though not so clearly in the time sequence in Figs. 3.213.23 .

These differences are not obvious and it is easy to see why the outflow influence hypothesis has been slow to gain acceptance. Because these differences are associated with variability in the asymmetric structure on large scales, it is not surprising that previous studies which concentrated on detecting differences in the symmetric transverse circulation were disappointing. Studies of tropical cyclogenesis by McBride and Zehr (1981) and the convective properties of developing cyclones by Arnold (1977) indicated that the mean upward motion inside $4^{\circ}$ radius was not systematically greater in developing systems, and that as development proceeded the total convective activity decreased. The role of favorable upper tropospheric outflow in stimulating the radial circulation was therefore opposed by Gray (1979), who hypothesized that intensification might be caused by a decrease in the radial circulation and thus a reduced export of moist static energy. From hydrostatic considerations, this energy increase would lead to warming and a pressure drop.

It has since been realized that intensification is associated with concentration of this pressure drop in the eye, and stimulation of the convective activity in the core region. A vortex may "Intensify" without "strengthening," and the associated change in symmetric 
transverse circulation be confined to radil of $100 \mathrm{~km}$ or less, inside the area resolvable by compositing studies. A study of the rainfall distribution in the core of Hurricane Allen by Marks (1985) indicated that the net rainfall within $1^{\circ}$ latitude radius of the center remalned nearly constant, but as the hurricane intensifled the rainfall became more concentrated near the center.

It is therefore crucial that the observed differences in Fig. 3.24 be related to changes in the core region if they are to influence intensity. Developing this concept requires an understanding of the structure and dynamics of the hurricane core, to be covered in sections 4.2 and 4.3 of the following chapter. Because the observed differences in radial wind are so small, the hypothesized lateral coupling between hurricane core and environment must be occurring through the asymmetric flow, the dynamics of which is discussed in sections 4.4 through 4.6 . The impact of the hypothesized asymmetric flow variability on the structure of the hurricane and its intensity is the subject of Chapter 5. 
4. SYMMETRIC AND ASYMMETRIC ASPECTS OF HURRICANE STRUCTURE AND DYNAMICS

\subsection{Historical Context}

As discussed in Chapter 1, conceptual thought regarding interactions between hurricanes and enviromental flows came to a near standstill with the development of CISK theory and numerical modeling efforts. A partial explanation of this was that no one consistent explanation of how the enviroment af fected the hurricane was accepted even among those who contended that it did. Investigators such as Riehl (1954) and Sadler (1978) stressed the importance of an "outflow mechanism" which would help "remove excess heat," while the widely known observation that vertical wind shear in the environment could weaken a hurricane (Lew1s and Jorgensen, 1978) or prevent its development altogether was explained as the "prevention of the accumulation of heat" by a "ventilation" process (Dunn and Miller, 1964; Gray, 1968, Riehl, 1979). When contrasted with the conciseness of CISK, the academic prestige of its proponents, and the quantitative results of modeling, it is no wonder that the environmental-effects concept was not warmly embraced by the research community. Axisymmetric representations of the hurricane are simpler, both dynamically and conceptually, than asymmetric ones, and the asymmetries are even more nearly intractable if they increase in amplitude with radius. The hurricane vortex was regarded as a complex enough research topic without encumbering it with surrounding flow variability if possible, and the 
asymetric structure has become the subject of determined study only in the past ten years, beginning with efforts to understand rain bands. The intent of this chapter is to develop a conceptual picture of hurricane structure and dynamics such that the purely observational conclusions of Chapter 3 can be interpreted physically. The simpler axisymmetric approximation to the hurricane will be explored first, by reviewing observations in section 4.2 and analytical models in section 4.3. Asymmetric features will then be introduced by a discussion of their bulk role in angular momentum transports (section 4.4), and their representation as wave motions in studies of spiral bands (section 4.5 ).

Section 4.6 combines the dynamical properties of waves in vortices as discussed in section 4.5 with a wave-mean flow decomposition of an outflow layer composite in rotated coordinates to describe the wave motion responsible for the diagnosed eddy import of angular momentum alluded to in section 4.4. Also discussed is the possible instability responsible for this wave, and the way in which this wave may connect the symmetric vortex structure at inner radil with the large-scale environment.

\subsection{Structure and Dynamics of the High-energy Core}

As discussed in Chapter 1, changes in hurricane intensity involve a very small region of the entire cyclone. Though this paper contends that intensification is influenced by the environment, the actual intensity changes occur within the high-energy core. This region of approximately $100 \mathrm{~km}$ radius contains the eye, the eyewall, and perhaps a rain band or two. It is structurally and dynamically very complex, but has also received the ilon's share of the research attention. Research alrcraft flights since 1957 have been specifically 
aimed at sensing core features, and numerous case studies and several more general treatments of the data (Shea and Gray, 1973; Gray and Shea, 1973; Willoughby et al. 1982, 1984, and Jorgensen, 1984) have resulted In a relatively complete description. Many of the features have been modeled, and theoretical work on the response of vortices to diabatic effects (Schubert and Hack, 1982; Shapiro and Willoughby, 1982) have led to a fuller conceptual understanding.

The most striking aspect of the structure of a mature hurricane is the degree of concentration. Fig 4.1 shows composite research flight legs (Shea and Gray, 1973); note that about one-half of the total pressure drop and temperature increase from periphery to center occur within the eyewall. The warmest temperatures and lowest pressures are found within the eye, and are associated with subsidence. Heat is released in eyewall convection and redistributed from eyewall to eye by a secondary circulation with subsidence in the eye and ascent in the eyewal 1.

The processes which maintain the subsidence in the eye have been debated for a long time. Malkus (1958) and Kuo (1959) attribute the subsidence to the existence of supergradient winds inside the eyewall in the low levels. Associated with the supergradient winds is an outward acceleration and a weak outflow from eye to eyewall. Continuity is satisfied by weak subsidence in the eye. The supergradient winds are maintained by an inward mixing of momentum across the eyewall. Composite analyses of flight legs (Gray and Shea, 1973) Indicate that supergradient winds are present while some individual cases (Hawkins and Rubsam, 1968; Willoughby, 1979b) indicate that near-gradient balance exists. Some supergradient winds would be expected in the region where 


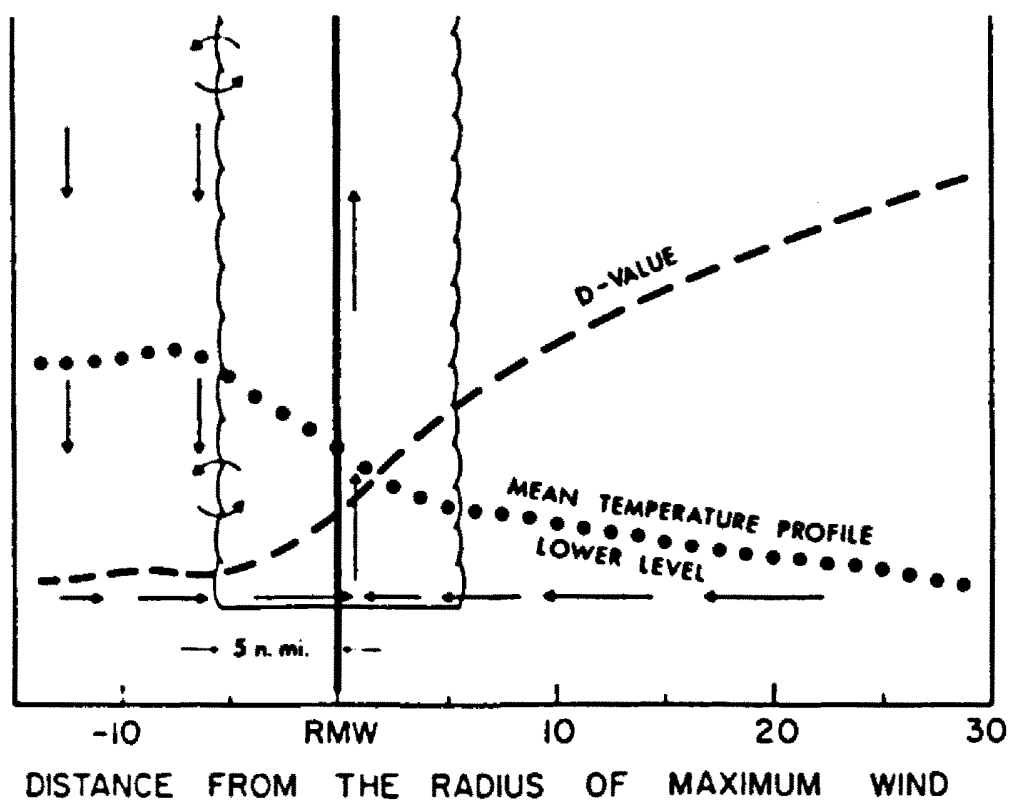

(n. mi.)

Fig. 4.1. Composite profiles of D-values, temperature and wind speeds relative to the radius of maximum wind, as measured by research aircraft. Figure from Shea and Gray (1973).

the inflowing air is decreasing in radial speed (outward acceleration) so even those cases which do Indicate supergradient winds may not indicate outflow. The maintenance of subsidence by a momentum mixing process is an adjustment of the mass fleld to the wind field forcing. which is unlikely in the high-rotation region of the hurricane core (Schubert and Hack, 1982).

An alternate explanation involving wind-to-mass adjustment has been proposed by Shaptro and Willoughby (1982). They studied the response of a balanced vortex to point ( $r$ ing, if the axisymmetry is considered) sources of heat, and found that for a high enough rotation rate, the horizontal scale of the response to the heating became smaller than the radius at which the heating occurs, leading to subsidence inside as well as outside the ring. Sufficiently strong subsidence would act to suppress convection within the ring, leading to the formation of an eye. Note that this is not a closed theory, related as it is to the specified 
location of the heating. However, axisymmetric, hydrostatic hurricane models using a convective parameterization that depends on low level convergence will produce an eye in this manner; Eliassen (1971) shows that frictionally induced vertical motion (Ekman pumping) decreases Inward near the center of rotation and convective heating would likewise decrease inward. At sufficiently high rotation rates (intensities), subsidence begins near the center (as explained by Shapiro and Willoughby, 1982) and an eye forms. The heating distribution in an actual hurricane is much more difficult to determine, but modifications of the heating structure are a likely way to induce an intensity change. Such was the aim of the Stormfury modification experiments, and modifications of the heating field must ultimately be included in a cyclone-environment interaction hypothesis.

The relationship between eye character and intensity change was discussed by Jordan (1961); intensifying typhoons have warmer, drier eyes and a lower temperature inversion, indicating stronger subsidence. To the extent that the concepts of Shapiro and Willoughby (1982) are correct, this is the result of increased or redistributed latent heat release or possibly a different rotational flow field resulting in a different response. The warming effect of the eye subsidence can also be of fset by a number of factors, such as turbulent mixing of warm air out of the eye, introduction of water condensate into the eye where evaporative cooling results, and radiative cooling. In a "steadystate" situation, the subsidence and these three dissipative effects are presumably in near-balance. Intensity change would result when any of the processes is enhanced or retarded, disturbing the balance. 
The above is a largely thermodynamic description of the balance which determines the eye temperature. From the energetic standpoint, the intensity of a hurricane is determined by a balance between the dissipation of kinetic energy in the eyewall with the production of avallable potential energy by latent heat release in the eyewall (assuming effective conversion from APE to $\mathrm{XE}$ ). The amount of latent heat is determined by the convergence of molsture (strongly controlled by frictional convergence in the core of a fully developed vortex) and by evaporation, which is determined by a poorly understood combination of surface wind speed, ocean temperature and surface conditions (related to drag and evaporation coefficients), and boundary layer drying by convective downdrafts. The latter in turn is related to the buoyancy of the updrafts and thus the intensity of the convection, making the whole situation highly nonlinear. The energetics do seem to impose some basic constraints, whlch were alluded to in the discussion of sea-surface temperature and will be discussed in detail in the next section.

\subsection{Thermodynamic Influences on Cyclone Intensity}

This section discusses a hierarchy of empirical and analytical models describing the thermal structure and intensity of a steady state hurricane. The first two models (Miller, 1958 and Riehl, 1963) describe environmental effects only qualitatively. The last (Emanuel, 1985, Lilly and Emanuel, 1985) explicitly relates intensity of a steady-state hurricane to a set of oceanic and atmospheric parameters including an "outflow temperature." Each model and its treatment of environmental effects will be briefly described in succession.

The earliest and simplest "model" is that of Miller (1958), who sought to estimate the character of eye soundings which could be 
developed from moist ascent and subsidence of surface air. Jordan (1952) contended that the high temperatures in the eye could be obtained only by subsidence from the upper troposphere, while the relatively high humidities found in the eye required the addition of water vapor via horizontal mixing. The air in the eye at a given level was considered to be a blend of eyewall air which had subsided from the upper troposphere, and eyewall air from that level which had laterally mixed into the eye. Miller (1958) assumed a surface parcel with temperature equal to the ocean temperature and a relative humidity of $85 \%$ and lifted such a parcel to $100 \mathrm{mb}$ to determine the eyewall sounding. The eye sounding was begun with eyewall air a $100 \mathrm{mb}$ and subsided dryadiabatically, with eyewall al $r$ mixed in at each level according to an empirical function of height. Assuming the mean helght for an undisturbed tropical atmosphere at $100 \mathrm{mb}$ and integrating the hypsometric equation yielded a surface pressure, which depended upon the initial sea-surface temperature. These results are shown in Fig. 3.12 . Miller considered the pressure so obtained to be a theoretical lower bound, and presented several cases of hurricanes which deepened to this pressure and then leveled off. Whether or not a given hurricane attained its potential was considered a function of the envirormental flow, particularly at $200 \mathrm{mb}$. Miller attempted to examine this influence by averaging daily $200 \mathrm{mb}$ height analyses for 5 hurricanes which reached the theoretical maximum intensity, beginning with two days prior to maximum and continuing to two days after maximum. The resulting series of 5 "dally" helght composites showed a trough to the northwest, a major high to the east, and a secondary high to the southwest of the hurricane center. Intensification was accompanied by a 
deepening of the trough and a building of the high to the east. A similar series of composites for 4 non-intensifying hurricanes revealed that the trough to the northwest and the high to the east were much less pronounced. Without wind composites the exact nature of the outflow is unknown, but Miller felt that the intensifying case developed outflow to the north.

Miller's attempt to estimate intensity involved a number of assumptions and empirical relationships. In particular, the moisture distribution (hence the subsidence and mixing) rates are known to be quite variable, particularly as the cyclone changes from intensifying to filling (Jordon, 1961). Later estimates of hurricane intensity use a more complete analytical model (Riehl, 1963; Emanuel, 1985) or fully nonlinear numerical simulation. (Anthes, 1982 provides a review and bibliography of this much-studied subject.) These have generally concentrated on the inner workings of the hurricane and have kept the "enviroment" (usually some specified outer boundary condition) as simple as possible.

The analytical model of Riehl (1963) uses the steady-state assumption to deduce the maximum wind which will satisfy angular momentum and energy constraints. The inflow is assumed to conserve potential vorticity, which for a bulk aerodynamic estimate of surface stress requires a tangential wind profile of $\mathrm{vr}^{1 / 2}=$ constant. Given a tangential wind in inflow and outflow layers at a fixed boundary radius, the angular momentum loss between inflow and outflow can be calculated, and the low-level wind profile integrated imward until the required loss accounted for. This specifies a maximum wind and radius of maximum wind for steady-state conditions. 
Another maximum wind estimate in the Riehl (1963) model is derived from energy considerations. The energy gain from the sea surface in the inflow is obtained from a bulk-aerodynamic formulation and the increase in $\theta_{e}$ from the boundary radius to the radius of maximum winds (as was estimated from momentum balance) is computed. This is then converted to a surface-pressure drop by using an empirical linear relationship between $\theta_{e}$ increase and pressure decrease, and the pressure decrease used to estimate the maximum wind attainable by KE generation. For steady state vortices, this estimate and that based on the angular momentum budget should be the same.

Though Riehl (1963) stresses the influence of enviromental factors on hurricane structure in the conclusions, the model does not explicitly contain enviromental effects. In order for the angular momentum constraint to be valid, eddy imports in the upper troposphere must be negligible. Angular momentum budgets (Holland, 1983) indicate that this is likely true only at inner radil. Because it imposes no environmental condition on the thermal structure and uses a linear relationship between $\theta_{e}$ increase and pressure drop, the Riehl (1963) model has no sea-surface temperature sensitivity at all.

The Emanuel (1985) model Includes some environmental effects explicitly by assuming an enviromental sounding (constant saturated molst static energy $h *$ in the troposphere and increasing $h *$ with height In the stratosphere) and matching the vortex structure to it. It also assumed that the model is axisymmetric and neutral to moist slantwise (along absolute angular momentum $M_{a}$ surfaces) convection, so that the $M_{a}$ and $h *$ surfaces in the vortex interior coincide, and that the moist thermodynamics are reversible. The latter is valid in regions of strong 
convection or mean upward motion. Under thermal wind balance, this allows calculation of the slope of an $\left(h *, M_{a}\right)$ surface; Integration of the slope with prescribed environmental thermal structure gives a relationship between $h *$ and $M_{a}$ gradients at the top of the boundary layer. Conversion from $M_{a}$ to pressure using gradient balance and integration from the "environment" (no rotation) yields a relationship between central pressure and central $h *$. By assuming 1sothermal Inflow, the explicit $h *$ dependence can be eliminated, ylelding central pressure as a function of $\left(T_{B}-T_{0}\right) / T_{B}$ where $T_{B}$ is boundary layer temperature and $T_{0}$ is outflow temperature) and $\mathrm{RH}_{s}-\mathrm{RH}_{a}$, (relative humidity at center and enviroment respectively).

Figure 4.2 shows Emanuel's "theoretical limit" on intensity, obtained by setting $\mathrm{RH}_{S}=100 \%$ and assuming an environmental pressure of $1015 \mathrm{mb}$ and $\mathrm{RH}_{\mathrm{a}}$ of $80 \%$ at $20^{\circ} \mathrm{N}$. Central pressures are obtained for a range of surface air temperatures and tropopause temperatures. Seasurface temperatures are assumed to be about $2^{\circ} \mathrm{C}$ higher than air temperatures in the isothermal inflow area. The warmer the surface and the colder the outflow temperature (same as the tropopause temperature for the isothermal stratosphere in the Emanuel model), the higher the potential intensity. The potential intensities using July mean conditions are shown in Fig. 4.3.

Emanuel also assumes a boundary layer with constant $\mathrm{RH}$ outside the radius of maximum winds and $\mathrm{RH}=100 \%$ inside and derives a complete two-dimensional structure. The model hurricane has a realistic structure except at outer radil and high levels, where the assumption 


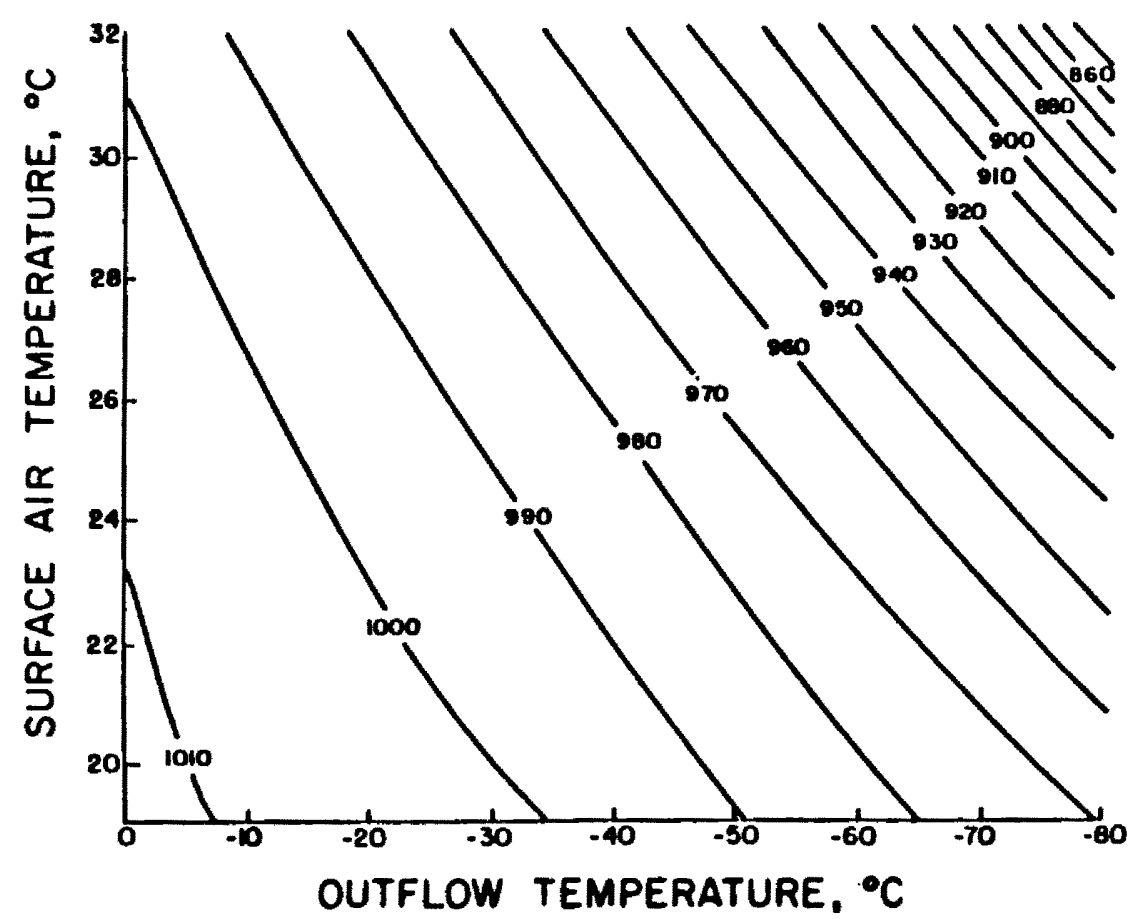

Fig. 4.2. Theoretical limit on intensity from Emanuel (1985) for $20 \mathrm{cN}$ latitude, enviromental pressure of $1015 \mathrm{mb}$, and environmental humidity of $80 \%$.

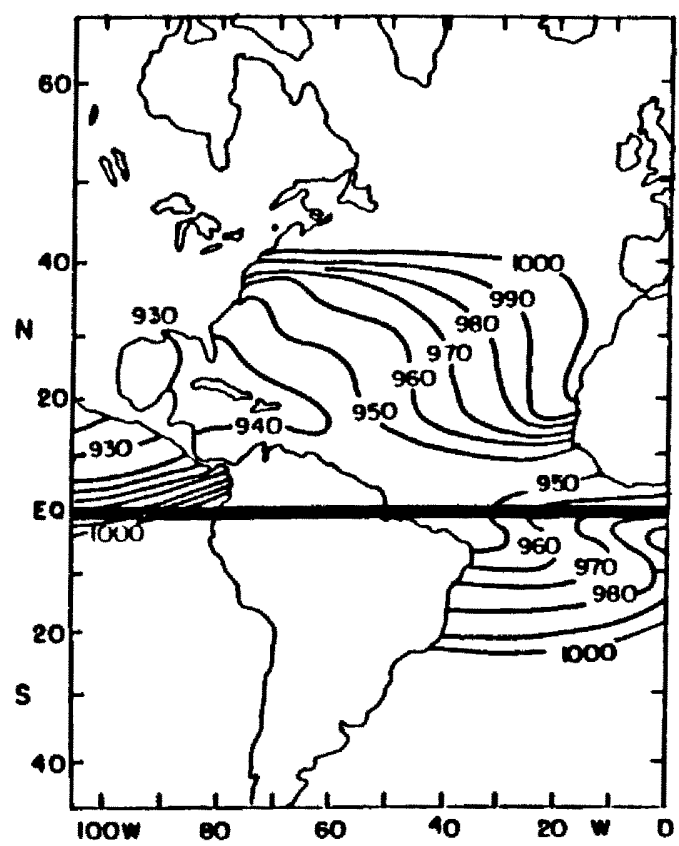

Fig. 4.3. Theoretical maximum intensities for July, based on climatological sea surface temperatures and tropopause temperatures. Figure from Emanual (1985). 
that the outflow extends to infinite radius along constant $\left(M_{a^{*}} h^{*}\right)$ surface leads to strongly anticyclonic winds and vertical shear. In reality, the outflow does not conserve $M_{a}$ because of the strong imports by eddy fluxes. Emanuel does not comment on this discrepancy, nor does he directly discuss cyclone-environment interactions, with the exception of the outflow temperature.

4.4 Effects of Asymmetric Processes on Symmetric Flow

All of the analytic models described above have dealt with the surface source of heat and sink of angular momentum and side-stepped the effect of asymmetries in the flow. This is not surprising since, unlike surface fluxes, they cannot be easily parameterized and their presence clutters an otherwise clean argument. Their effects can be deduced from observations as a residual in the angular momentum budget, and the role of eddy fluxes in the angular momentum balance of hurricanes has been a topic of research since Palmen and Riehl (1957) and Pfeffer (1958). Holland (1983) contains an excellent discussion of angular momentum transports. Eddy fluxes of momentum are associated with spatial correlations in the asymmetric radial and tangential winds. If areas of Inflow tends to also be areas of cyclonic flow and outflow tends to be associated with anticyclonic flow, the effect of flow asymmetries will be to import angular momentum. Such is the case at synoptic scale radil around hurricanes in the upper troposphere.

Observations of large values eddy angular momentum imports in the upper troposphere surrounding tropical cyclones by Frank (1977b), and of eddy flux differences between developing and non-developing tropical disturbances (McBride, 1981), led Challa and Pfeffer (1980) and Pfeffer and Challa (1981) to study the effects of eddy momentum fluxes on an 
axisymmetric model. The magnitude of eddy flux divergence was specified as a "forcing" in the transverse circulation equation of the balanced vortex model of Sundqvist (1970). Using observed magnitudes of flux divergence, a wide range of cyclone development rates and characteristics were obtained depending upon the vertical distribution of the flux, pointing to the influence exerted by "environmental effects".

Two criticisms of the above research can be made. First, the specified eddies transported only momentum and not heat. Depending upon the eddy heat flux convergence, different symmetric responses could be had for the same momentum transports. Second, the very idea of "specifying" an eddy flux is suspect, particularly when asymmetric models initialized with an axisymmetric initial condition later develop asymmetric flow and eddy fluxes on their own from barotropic or inertial instability (Anthes, 1972; Kurihara and Tuleya, 1974). St1l1, the degree of cyclone variability associated with the specified eddies Indicates that they are a significant factor.

The effect of the eddies on the angular momentum budget is relatively easy to deduce. What is more difficult to understand is the structure and dynamics of the eddies themselves. Some progress has been made in the area of wave dynamics for vortex basic states in an attempt to explain rainbands, and the rotated compositing method allows the wind structure of upper-tropospheric asymmetries to be isolated. These two approaches will be drawn together in the remalnder of this chapter to describe the upper tropospheric wave associated with eddy transports and hurricane-environment interactions in the upper troposphere. 


\subsection{Review of Wave Properties in Vortices}

The dynamics of asymmetric perturbations in hurricanes have been treated mathematically in the past 10 years by Kurlhara (1976), W11loughby $(1977,1978 \mathrm{a}, \mathrm{b})$, and Schubert (1985). Host of the work on the dynamics of waves in an axisymmetric basic state has attempted to provide an explanation for spiral rain bands, which are often apparent In radar returns from hurricanes. They typically occur on scales of a few hundred kilometers or less, and on time scales ranging from a few hours to a day. The dynamics of spiral-shaped perturbations has been Investigated by Kurihara (1976) and W1lloughby $(1977,1978 \mathrm{a}, \mathrm{b})$. A consensus that the spiral bands are inertia-gravity waves has arisen, al though the way in which such waves are excited and the direction in which they propagate is still the subject of Investigation.

The method of Kurihara (1976) is the application of linear perturbation analysis to an axisymmetric basic state. Perturbations which are sinusoidal in time, azimuth, and radius are then assumed and for specified radial and azimuthal wave numbers. Solution of an elgenvalue problem ylelds frequencies, growth rates, and vertical structures for various wave types (clockise or counterclockwise spiral) and modes (imward propagating inertia-gravity, outward propagating inertia-gravity, and geostrophic modes). He sought those types and modes which had positive frowth rates as explanations for rain bands, and concluded that the outward-propagating gravity wave best fitted observations and results from an asymmetric model (Kurihara and Tuleya, 1974). The imward-propagating gravity wave and a geostrophic mode were also considered dynamically possible, but Kurihara (1976) was unaware of any observational evidence for their existence. 
Willoughby (1977, 1978a, b) adopted a different approach. Rather than assume a perturbation structure and solve for a complex frequency, he assumed a steady-state forcing at specified frequency and radius location and solved for the radial structure. Instability (growth of waves) is then manifested as a radial divergence of wave energy flux. Willoughby's analysis was unable to support a propagating geostrophic mode (he attributes this to the "rapid horizontal variation of the mean vortex", Willoughby et al., 1984). Figure 4.4 shows some of the properties of the inertia-buoyancy wave which Willoughby (1978b) feels often responsible for banding in hurricanes. Willoughby (1979a) proposes that these waves are excited by superposition of the vortex on an environmental current with horizontal shear, resulting in a wavenumber 2 forcing. Isolation of these waves in observations has proven difficult, al though Willoughby (1978a, 1979b) presents evidence of this wave type using both land based radar and aircraft-measured wind observations.

The "geostrophic" mode (Kurihara's "F-mode" wave) has received far less theoretical attention than has the inertia-buoyancy type, al though it (and its effects) are easier to observe and it is more clearly an enviromentally-dependent feature. Tuleya and Kurihara (1984) describe the formation of "comma bands" under the influence of the $\beta$-effect and enhanced surface fluxes. Anthes (1982) cites several references describing stationary bands, and Willoughby et al. (1984) have recently defined a "ștationary band complex" (SBC) that is distinct from the propagating bands which are attributed to gravity waves. The $\mathrm{SBC}$ is located outside the high wind region and, using Willoughby's (1978a) pass band criterion, is of too low frequency to be 


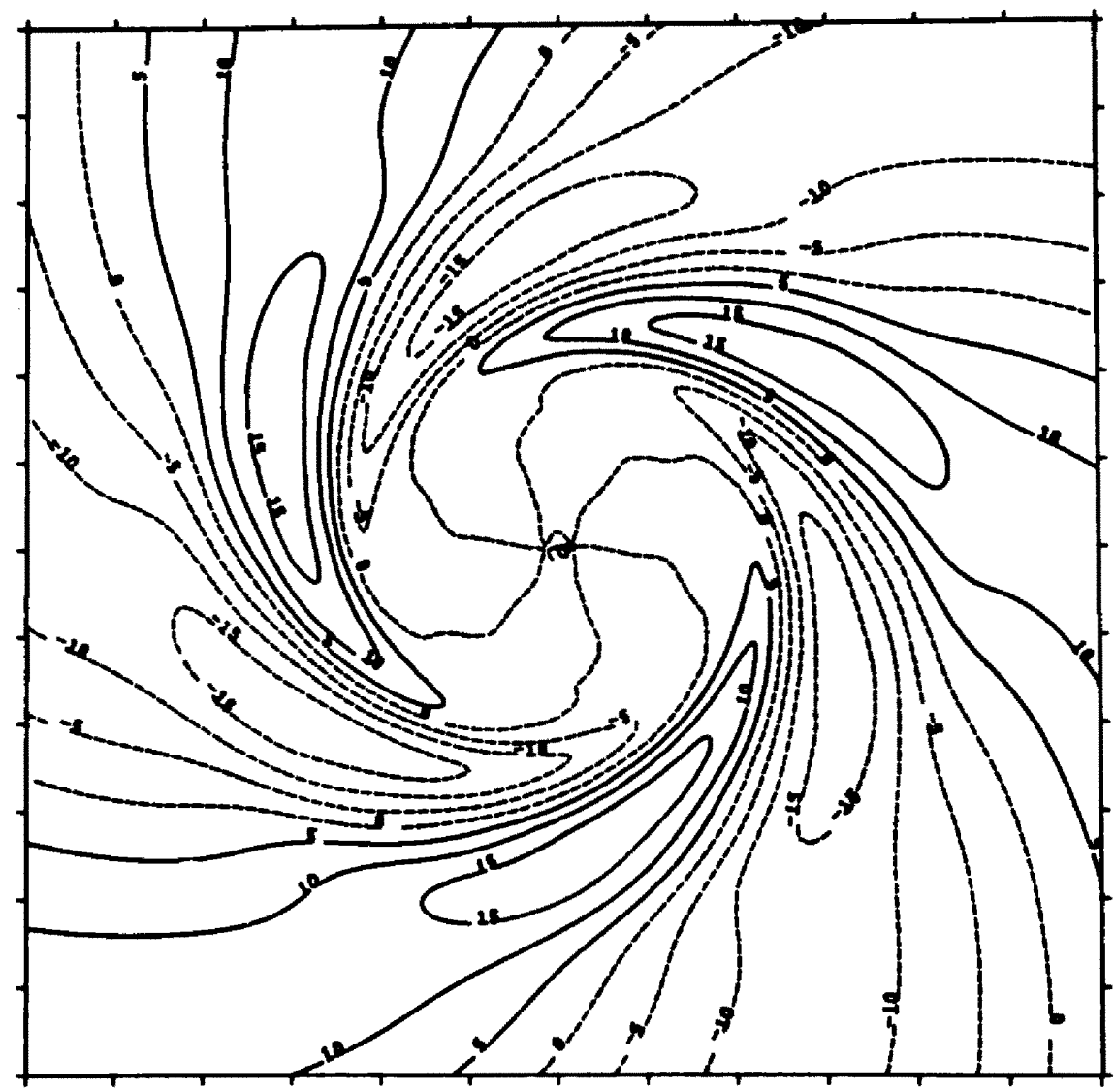

Fig. 4.4. Horizontal geopotential structure (in nondimensional units) of a wavenumber 3 inward propagating inert1a-buoyancy wave, at a helght of around $600 \mathrm{mb}$. Tick marks on axes are every $1 / 2$ non-dimensional length unit, with non-dimensional length scale equal to the radius of maximum winds. Note the gradual increase in amplitude as the wave propagates inward, and the wave absorption at the radius of maximum winds. Adapted from Fig. 7 of Willoughby (1978b).

an inertia gravity mode. Willoughby et al. (1984) attribute the existence of the stationary "principal band" to the "bow shock" of the mid-level environmental flow impinging on the vortex as it moves in a vertically sheared environmental current. The formation of an SBC by this process will be described in Chapter 5 in connection with the influence of vertical shear on hurricane intensity.

MoDonald (1968) hypothesized the existence of a "Rossby-mode" wave as being the hurricane analog to tilted troughs in the westerlies 
of the Earth's general circulation. Figure 4.5 shows the similarities between Rossby-mode waves in three flow types: the Earth's general circulation, a cyclonic vortex, (described by McDonald, 1968) and an anticyclonic vortex (discussed in the next section). These waves are described as "Rossby mode" because they all propagate (move relative to the basic flow) by advecting the vorticity of the basic flow. The wave trough (a relative vorticity maximum) will propagate towards the area in which the advection of basic state vorticity (Corlolis parameter for the planetary circulation, relative vorticity of the symmetric mean flow for the hurricane flows) is positive. This propagation mechanism is shown at lower left in Fig. 4.5. If the axis of this wave type is "tilted" in the horizontal (not perpendicular to the basic flow), the wave can transport angular momentum and possibly interact with the basic flow. McDonald (1968) sought observational evidence for this wave mode in the low levels using the NHRP flight data, but his results were inconclusive. The work of Wel and Gray (1985) does indicate that large scale cloud bands are associated with a vorticity maximum in the low level flow (characteristic of a Rossby mode), and with eddy angular manentum imports (associated with axis tilt), but such cloud bands are apparently not essential to tropical cyclone maintenance or intensification as many cyclones lack them al together.

All of the work above is directed at explaining rain bands, and is concerned with the flow asymmetries in the low-level vortex. However, we are interested in the structure of the upper tropospheric asymmetries which are hypothesized to be involved with environmental influences on hurricane intensity, as well as other aspects of hurricane structure and dynamics. Observational studies of the tropical cyclone by Frank 

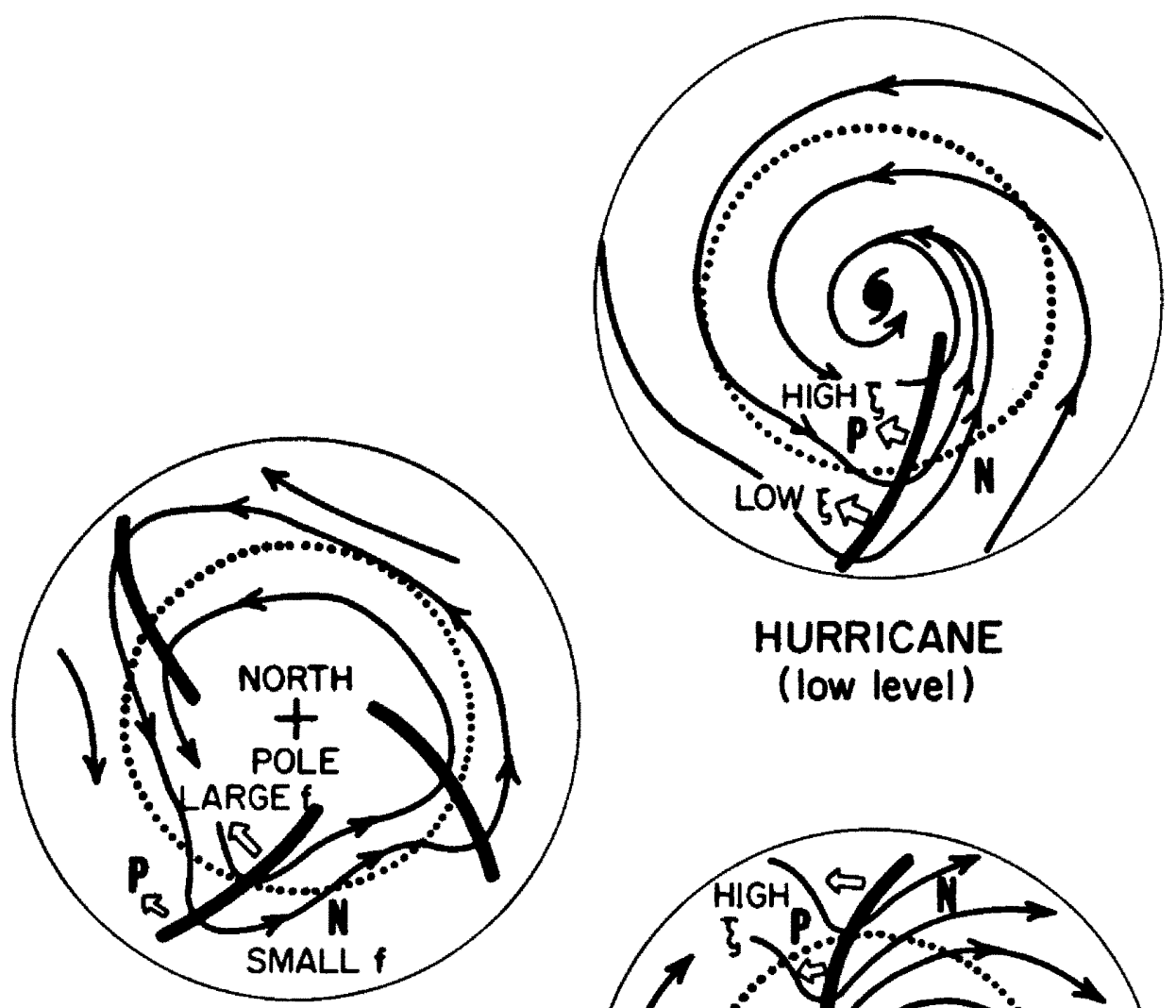

\section{PLANETARY CIRCULATION}
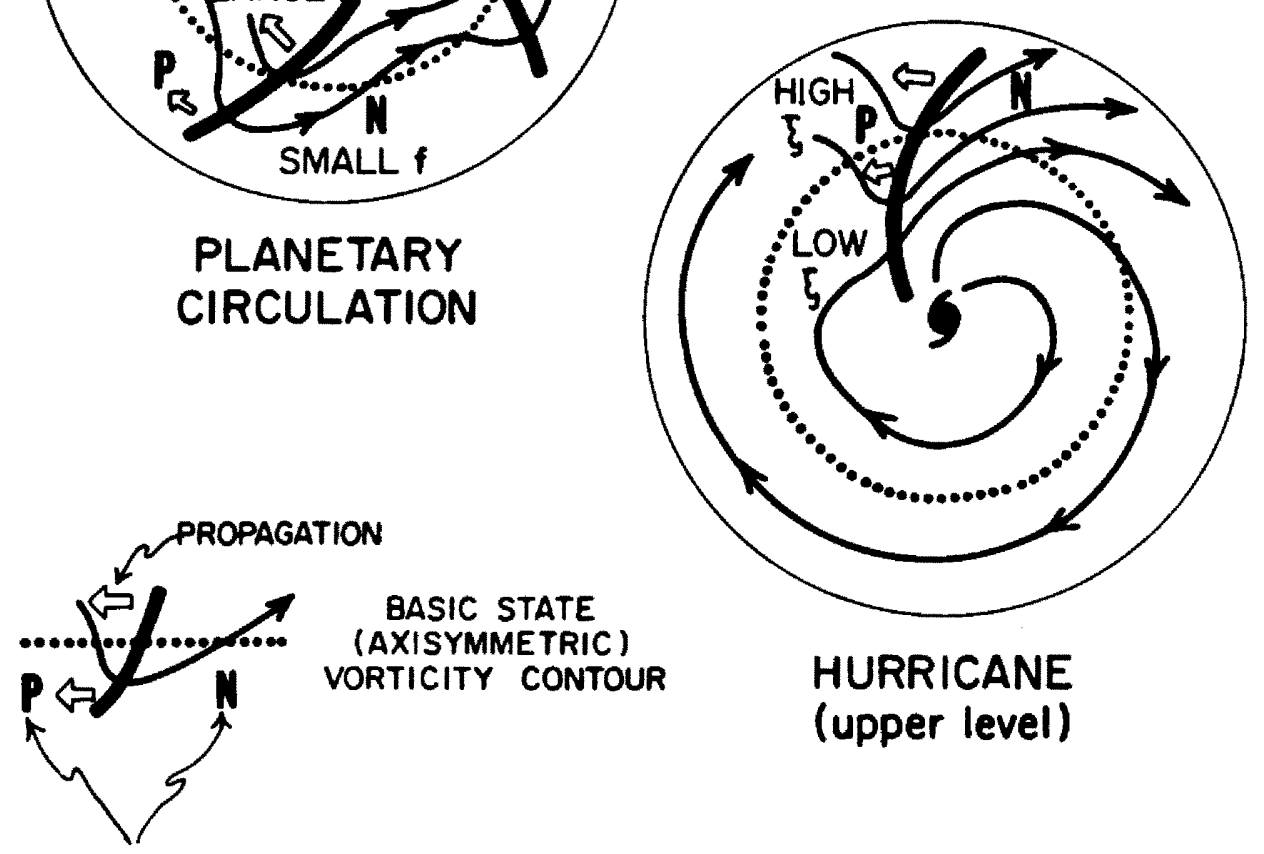

HURRICANE
(upper level)

BASIC STATE VORTICITY

ADVECTION (PVA Or NVA)

BY WAVE

F1g. 4.5. Planetary (left) and hurricane (right) "Rossby Waves". All waves propagate against the basic current direction and are nearly stationary. Wave axis tilt is associated with angular momentum flux $\left(\bar{u}^{\prime} v^{\prime} \neq 0\right)$; flux is Imward for the Planetary Circulation and upper level Hurricane cases and outward for the low level Hurricane. 
(1977b) and Holland (1983) Indicate that eddy processes are more important in the upper troposphere than in the lower troposphere. Modeling studies by Anthes (1972) and Mathur (1975) have al so shown that, through interactions with latent heating, asymmetries in the condensation assoclated with upper level vertical motion fields can apparently modify the low and mid levels sufficiently to cause the formation of deep convection which in turn generates a perturbation in the low level flow. Given these findings, and the assertion of Holland and Merrill (1984) that the upper troposphere is the primary region of cyclone-environment interactions, it seems worthwhile to discuss the wave propagation conditions for an anticyclonic basic state as is found in the upper troposphere. The "Rossby" mode expected in an anticyclonic basic state having an outward increase in relative vorticity is shown schematically in Fig. 4.5 at lower right. Inertiagravity modes may also be present in such a basic state. Unfortunately, none of the theoretical studies described above addressed the problem of anticyclonic flow, and this remains an area where research is needed. In the meantime, a purely observational approach will be followed. Using the rotated coordinate polar composites described in Chapter 3 , the "basic state" and "wave" making up the upper-tropospheric flow of hurricanes can be resolved and some of their properties described. 4.6 Characteristics and Observations of Waves in the Upper Troposphere The only direct examination of the asymmetric flow component of the hurricane outflow was undertaken by Black and Anthes (1971). They used satelite derived winds and rawinsondes to prepare outflow layer analyses for 4 individual cases. The "asymmetric" wind was then 
isolated by subtracting the mean radial and tangential wind at each $1^{\circ}$ belt in radius and analyzing the residual. Harmonic analysis in azimuth Indicated that nearly all of the asymmetry was at wavenumber 1 and 2 . The wavenumber 1 flow appeared as a unidirectional current oriented generally in the direction of the cyclone motion (not surprising since the cyclone motion was not removed from the winds). Wavenumber 2 consisted of a pair of eddies, anticyclonic to the left of the motion and cyclonic to the right. The eddy structure was quite variable, but the eddies were usually centered at about $6^{\circ}$ radius. Black and Anthes Interpreted the eddies as a result of a uniform current (environmental flow) streaming around an obstacle (cyclone). Their analyses did not extend past $8^{\circ}$ radius, so they were unable to see the larger-scale asymmetries.

Application of a similar analysis method to composites described in Chapter 3 allows the analysis of the asymmetric wind out to a larger radius of $15^{\circ}$ latitude. The asymmetry is partial ly preserved during the compositing process by the coordinate rotation. Figures 4.6 and 4.7 show the total and asymmetric streamlines and isotachs for the MOToutflow ROT composite for the "all-hurricane, no subtropical origin" composite from Chapter 3 .

Figure 4.7 clearly shows the asymmetry of the type described by Black and Anthes (1971), with a trough to the left and a ridge to the right of the cyclone motion which is towards the north-northwest. However, their hypothesis that this asymmetry is a result of the flow separation around a divergent core region apparently does not apply since the wavelike structure becomes more pronounced with increasing 


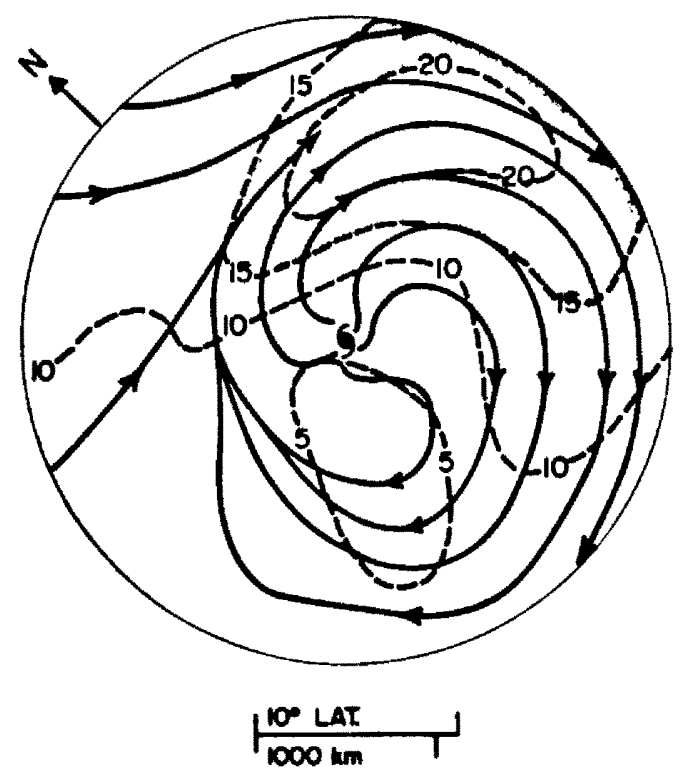

Fig. 4.6. Resultant winds $(\mathrm{m} / \mathrm{s})$ for the OROT composite of Atlantic hurricanes. The location of the mean outflow rotation angle is at the top of the grid. The cyclone motion vector was subtracted from each wind before compositing, so the wind field shown is relative to the moving system.

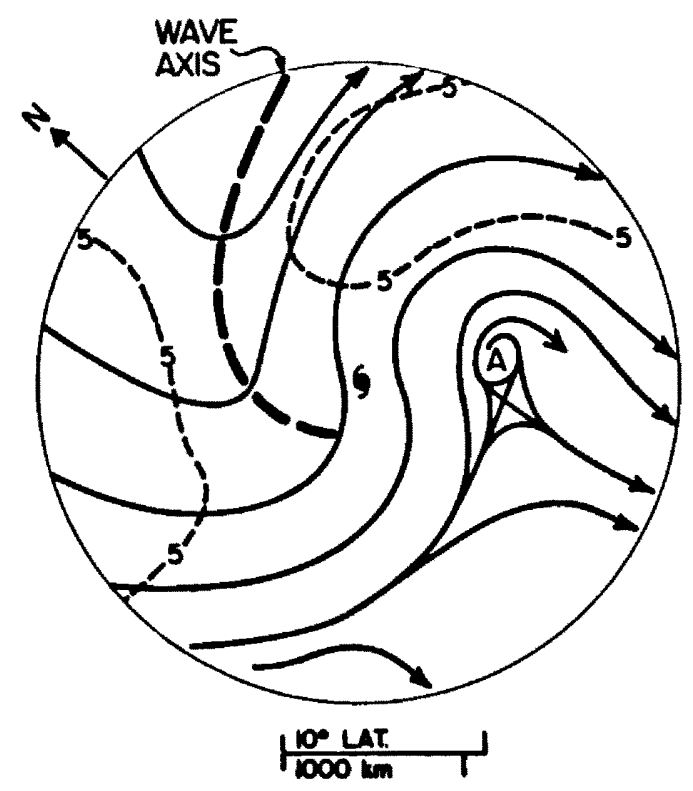

Fig. 4.7. Asymmetric flow component of Fig. 4.6 obtained by subtracting the belt mean radial and tangential wind components from the octant values, and recombining the modified octant values to generate vectors. 
radius out to $15^{\circ}$, implying that it is a characteristic of the largescale env1roment.

If we examine the radial structure of the basic state (Fig. 4.8) and the eddy momentum fluxes of the wave (Fig. 4.9) in addition to the plan view (Fig. 4.7), it is possible to deduce its dy namic properties, at least in part. The solid line in Fig. 4.8 shows the basic state tangential wind as a function of radius. The large-scale anticyclone, with maximum flow tangential speeds at radil of order $10^{\circ}$ latitude $(1100$ $\mathrm{km}$ ) is characteristic of fully developed tropical cyclones (Frank, 1977a), and some weaker systems as well (McBride, and Zehr, 1981). The dotted line in Fig. 4.8 shows the radial distribution of relative vorticity of the basic flow. Note that from $5^{\circ}$ to $12^{\circ}$ latitude (about $500-1300 \mathrm{~km}$ ) radius the vorticity increases outward so the basic state is the same as for the "Hurricane, upper level" situation in Fig. 4.5. For the axis tilt shown in Fig. 4.5 and observed in Fig. 4.7, inflow is associated with weaker anticyclonic flow than is outflow so the wave acts to import angular momentum, as is observed in the budget studies desoribed in section 4.4 .

Note that (on an $f$-plane) the necessary condition (Kuo, 1949) for barotropic instability

$$
\frac{d \overline{\xi_{a}}}{d r}=0
$$

where $\bar{\xi}_{a}$ is the basic flow absolute vorticity, is satisfied at a radius of $4-5^{\circ}$ latitude $(500 \mathrm{~km})$ as is apparent in Fig. 4.8. Barotropically unstable waves amplify at the expense of the kinetic energy of the mean flow, and would be associated with an import of angular momentum (reducing the anticyclonic flow of the basic state). Figure 4.9 shows the eddy momentum transports from the composite, indicating an eddy 


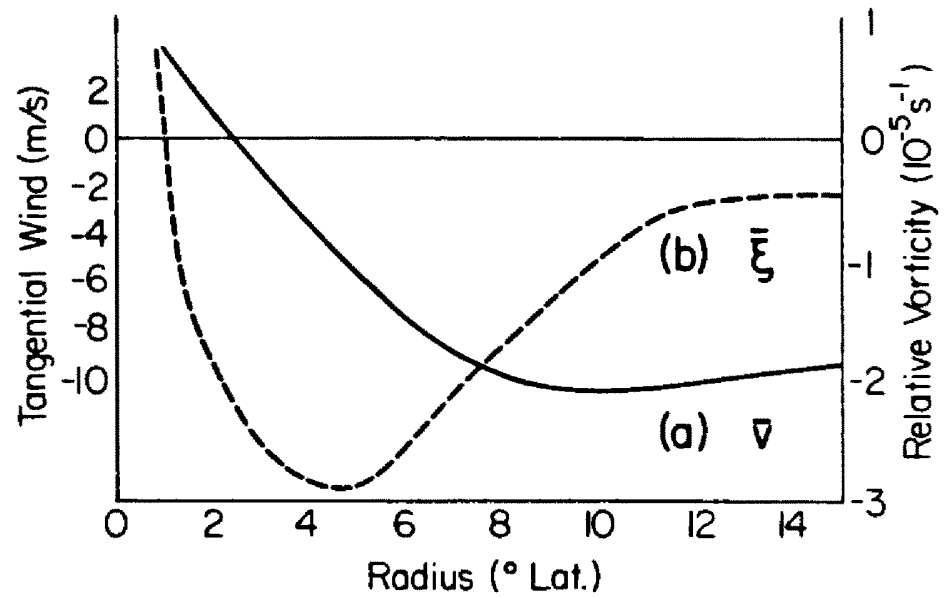

Fig. 4.8. Basic state (axisymmetric) properties of the flow in Fig. 4.6. (a) tangential wind, and (b) relative vorticity.

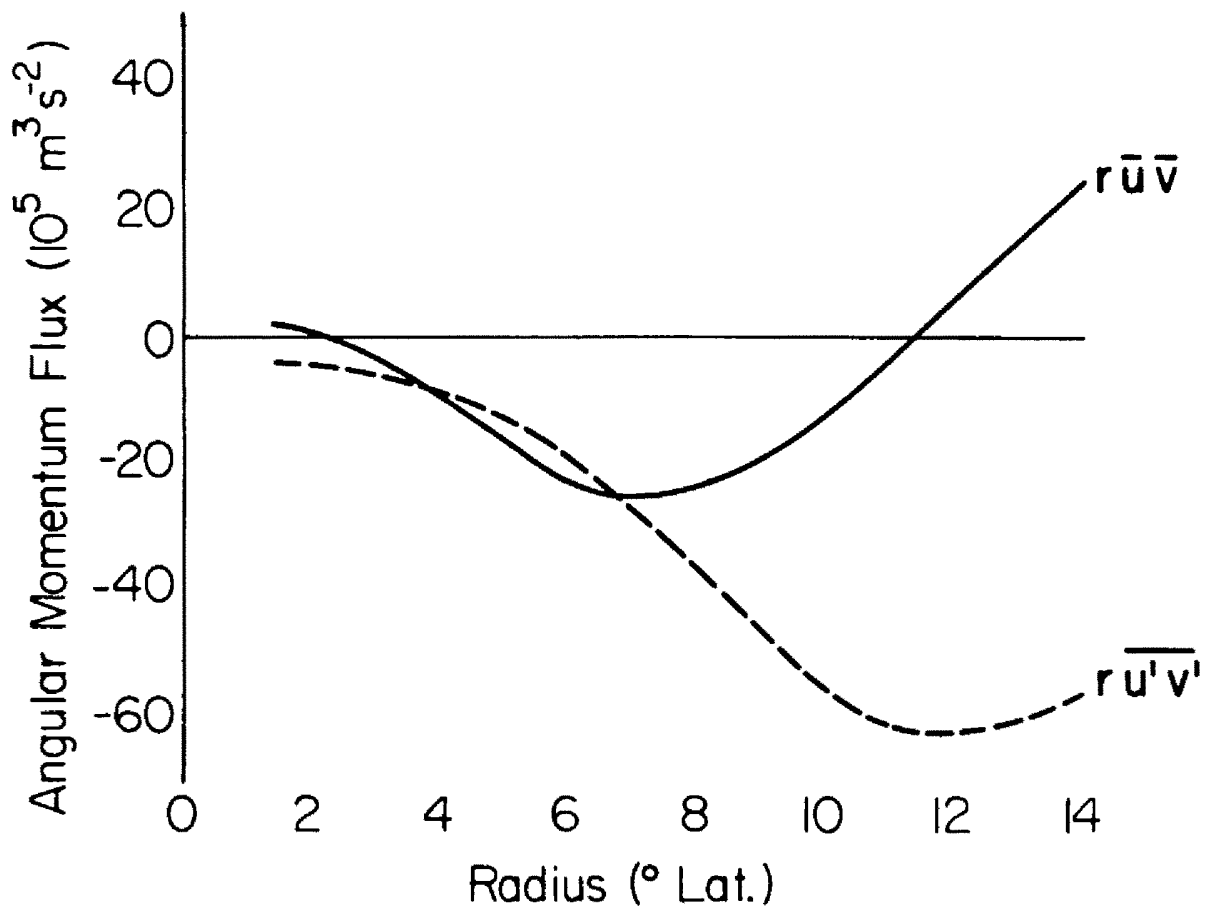

Fig. 4.9. Transports of relative angular momentum by mean (solid) and eddy (dashed) processes. Note the convergence of cyclonic momentum by the eddy processes, indicating mean flow rotation destruction and wave amplification. 


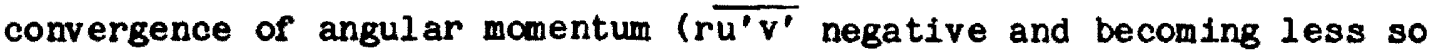
with decreasing radius) which would tend to reduce the mean flow, as is expected if barotropic instability is occurring. As is discussed by Schoeberl and Lindzen (1984), the "instability" problem becomes more complex as the waves amplify and nonlinear effects begin to af fect the basic state. The wave and mean flow are hypothesized to be nearly in balance, with the wave acting to destroy the instability as fast as the mean flow processes (diabatic heating and axisymmetric secondary circulations) are acting to put it back. This wave is proposed as the bridge between the cyclone scales at $500 \mathrm{~km}$ radius and less, and the envirorment at radil of $1500 \mathrm{~km}$ or more.

The existence of outflow asymmetries has long been recognized and their simulation was a target of the first three-dimensional hurricane models. The first reported asymmetric model was that of Anthes (1972), followed by Kurihara and Tuleya (1974) and Jones (1977). All were Initialized with an axisymmetric vortex in a "still air" environment and, as the vortex developed, areas of barotropic and/or inertial instability developed within the axisymetric, divergent flow in the outflow layer. These regions broke down into asymmetries, which grew by extracting kinetic energy from the mean flow, which was in turn maintained by the diabatic processes. Unlike those in Fig. 4.9, these eddies were largely confined to a radial band from $100-300 \mathrm{~km}$ radius, and decayed in amplitude away from the region of instability surrounding the convective heating. Even these Iimited asymmetries were important in the intensification of the model hurricane. Anthes (1972) reported a lower central pressure for the asymmetric case than for a symmetric 
control integration. It is felt that the influence exerted by the larger-scale atmospheric asymmetry is at least as great.

The "real" situation is depicted schematically in Fig. 4.10. Whereas the classical barotropic instability problem is solved subject to boundary conditions of zero perturbation amplitude in the meridional limits (very small and very large radius in cylindrical geometry), the "real atmosphere" condition is one of relatively large wave amplitude at large $r$ corresponding to the surrounding "environmental flow". Wavenumber 1 would be associated with a unidirectional flow, wavenumber 2 with uniform horizontal shear (part of which would also project onto wavenumber 0 - the basic state) and various troughs, ridges, and nonlinear horizontal shears as higher wavenumbers. The amplitude and time changes of these "boundary conditions" would have an influence on the structure of the wave assoclated with the barotropic instability at smaller radi1, as would the symmetric processes of diabatic heating, and vertical momentum transports acting to produce the barotropically unstable basic flow. An enviromental influence would occur when the "boundary condition" changed sufficiently to al ter the wave activity near the center, which would in turn alter the mean flow and possibly the diabatic heating rate and/or distribution. Evidence for and the implication of such a process will be discussed in detall in the next chapter, as will a proposed series of numerical experiments to simulate these processes. 


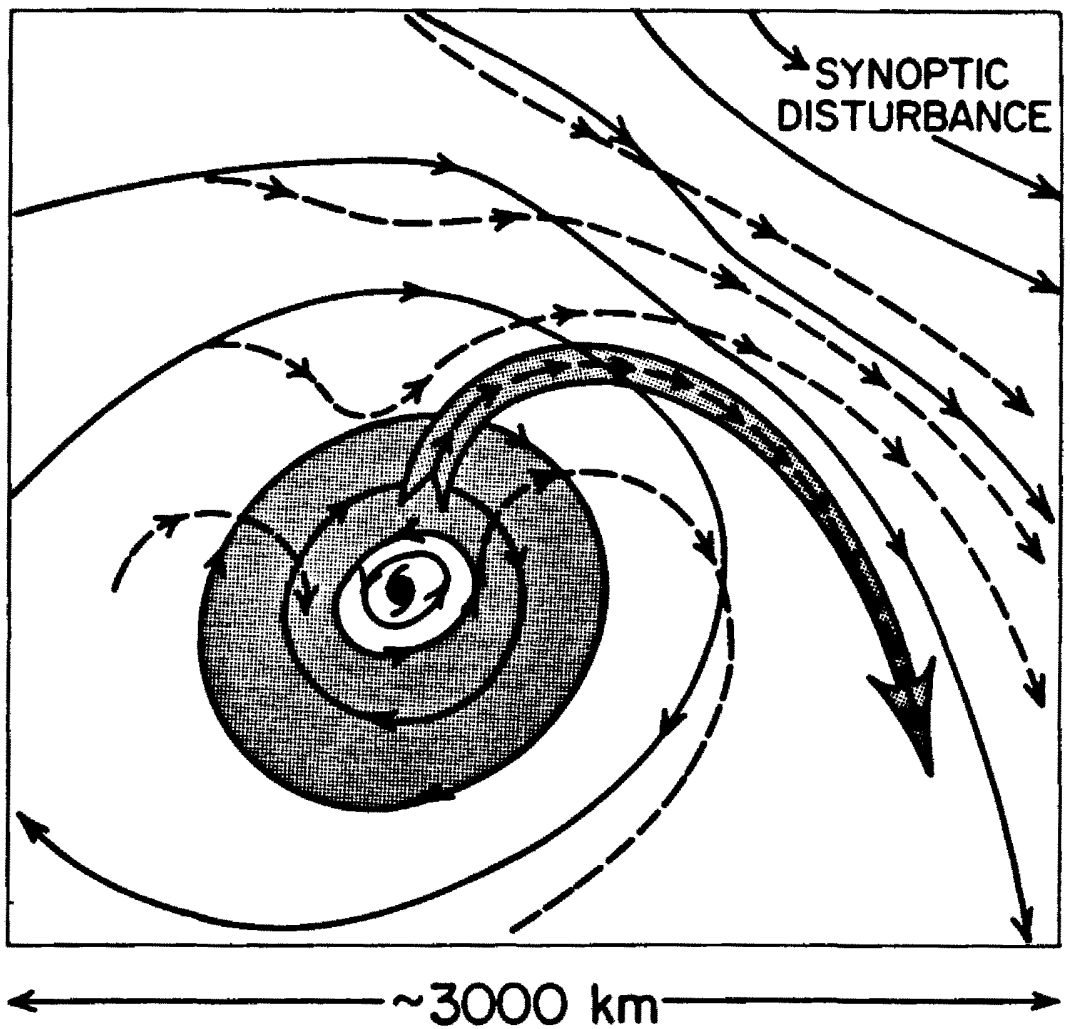

Fig. 4.10. Schematic of cyclone-enviroment interaction in the outflow layer. The solid streamlines are the environmental flow, which would be occurring whether the cyclone was there or not. Dashed streamlines are the alteration of the imposed environmental flow by barotropic instability (within the shaded area) of an axisymmetric basic state (the cyclone) maintained by diabatic heating. The outflow jet (large, shaded arrow) is a result of both the environmental effects and the presence of the cyclone. 


\section{DISCUSSION}

It was stated in Chapter 1 that the purpose of this paper was to re-examine hurricane intensity change in light of a new observational study of the upper-tropospheric environmental flow, assimilating wherever possible the results of other research. The latter task is especially important since most previous papers have tended to emphasize one particular process (cyclone-convection interactions, outflow structure, ventilation, convective asymetries, etc.) to the apparent, though hopefuldy not intentional exclusion of all else. The situation is still as described in 1947 by Norton:

\footnotetext{
- At present the situation regarding hurricanes is very much in the state of the blind men who went down to the circus to see the elephant".
}

The mechanisms which are thought to be important depend on who you ask and the data or model structure they study. This paper emphasizes the outflow layer structure in what is hoped to be a fitting compensation for years of willful neglect, but if there is one general conclusion it is that the "problem" of hurricane Intensity change cannot be simplified to a single mechanism; even a "simple", "qualitative" description must include a variety of effects and influences. It is the purpose of this chapter to discuss recent findings regarding the Internal structure of hurricanes, and to develop a "simple, qualitative" description of how these internal structures and the intensity might be influenced by the enviromental factors discussed in 
Chapters 3 and 4 . Ways in which this conceptual model can be tested are then stated, and the operational implications discussed.

\subsection{Internal Dynamics and Intensity Change}

The vast majority of papers on hurricane intensity change have focused on the structure of convection and precipitation and its relationship with the vortex circulation. The historical importance of the Idea that cyclone and convection cooperate is discussed at length in Chapter 1. So much has already been written about this subject that little more will be sald here, except for some thoughts concerning relationships between convective organization, overall low level circulation strength, and intensity change rates.

Since 1977, the specially equipped aircraft operated by NOAA have slowly built a "library" of observations of the mass, wind, and precipitation structures within the high-energy cores of Atlantic hurricanes at low levels. These observations have been generalized in papers by Willoughby et al. (1982), Jorgensen (1984), and Willoughby et al. (1984). The first discusses what is known as the "concentric eye phenomenon", and the second and third contrast the precipitation and wind structures of "symmetric" and "asymmetric" hurricanes. The manner in which these core-region characteristics may be related to environmental conditions is considered below.

Figure 5.1 shows schematically the radar return from the Stationary Band Complex (SBC) of an asymmetric hurricane and how a tropospheric enviroment with vertical shear acts to establish 1 , as described by W1lloughby et al. (1984). In Fig. 5.1, the hurricane is embedded in increasing westerlies with height. LOW level envirormental air impinges upon the hurricane vortex from the east (relative to the moving vortex) 

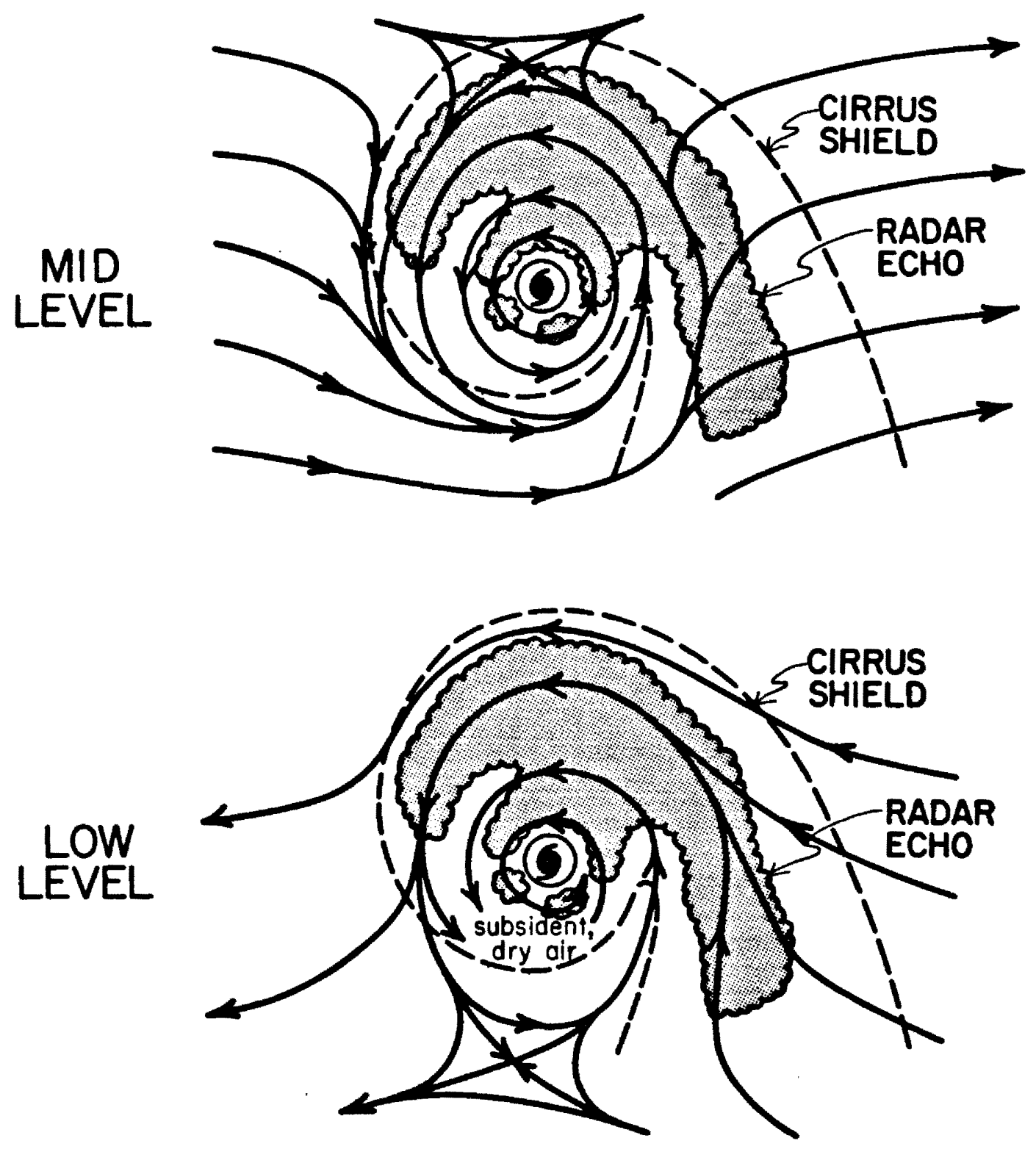

Fig. 5.1. Schematic representation of an asymmetric hurricane with a "principal band" (Willoughby et al. 1984) and the vertically sheared flow in which it is embedded. 
and converges, driving the principal band. Downstream (west) of the vortex, the low level flow is divergent. In the mid and upper troposphere, the situation is reversed, with divergence overlaying lowlevel convergence, general ascent, and the principal band to the east of the vortex. Likewise, the west side is dominated by convergence aloft and subsidence. Some of this sinking, dry air is drawn back into the core, where it begins regaining energy from the sea surface as it streams towards the eyewall. For strong shear, the descent on the down-shear side is more pronounced, and, al though the rate of surface flux may be quite large, no individual parcel entering the eyewall can acquire a high $\theta_{e}$ value because of the recycling by the asymmetric vertical motion fleld. If the shear is weaker, the asymmetric recycling decreases and inflowing air can attain higher $\theta_{\theta}$ before reaching the eyewall and a greater Intensity can be reached. This may be the manifestation of the "ventilation" process of intensity suppression described by Simpson and Riehl (1958). Total mass flux, surface flux, and latent heat release may be large and quite effectively supported by an efficient outflow but the intensity reduced by shear; a sheared current from the surface to $300-400 \mathrm{mb}$ results in an asymmetric precipitation structure and prevents the generation of a sufficient amount of high $\theta_{e}$ alr to maintain an intense system. As has been demonstrated analytically by Schubert (1985, personal communication), kinetic energy generation is most efficient when the latent heating is occurring in the core, in association with high inertial stabilities and, presumably high values of $\theta_{e}$ as well.

In addition to shear-induced asymmetry, another mode of intensity suppression, the concentric eye phenomenon (Willoughby et al., 1982) is 
observed by aircraft in symmetric, intense hurricanes. According to the discussion above, symmetric hurricanes are presumably in an environment with weak vertical shear so another explanation must be sought. The physical mechanism for the concentric eye phenomenon is based upon experiments using axisymmetric, balanced diagnostic models (Shapiro and Willoughby, 1982). Convective rings tend to contract with time, and it is thus proposed that an outer eyewall, once formed, will gradually contract until its associated subsidence destroys any pre-existing inner eyewal1, resulting in interrupted intensification or even a period of filling. This concentric eye phenomenon has been observed in most Intense Atlant1c hurricanes (W1l loughby et al., 1984). The question has been, "What initiates the outer ring?" Willoughby et al. (1982) suggested symmetric instability in the upper levels or gravity wave excitation, but without specifically considering environmental effects. Recent experiments with an axisymmetric, nonhydrostatic hurricane model (Lord et al., 1984) have suggested that downdrafts aided by 1ce-phase microphysical processes may also be involved. It seems that the assumption that the process is purely internal has been made, and that the possibility of an enviromental influence has not even been considered as an hypothesis.

Given the vertical-radial structure of $\theta_{e}$ as observed by aircraft and diagnosed by Emanuel (1985), a mechantsm by which convection at different radil may be selectively favored can be inferred. Figure 5.2 shows schematically the axisymmetric $\theta_{\theta}$ structure of a mature hurricane and a hypothetical "enviromental influence" In the upper troposphere and lower stratosphere. To the extent that outflowing air does not mix and therefore conserves its $\theta_{\theta}$, the enviroment must be able to support 


\section{HURRICANE ENVIRONMENT}

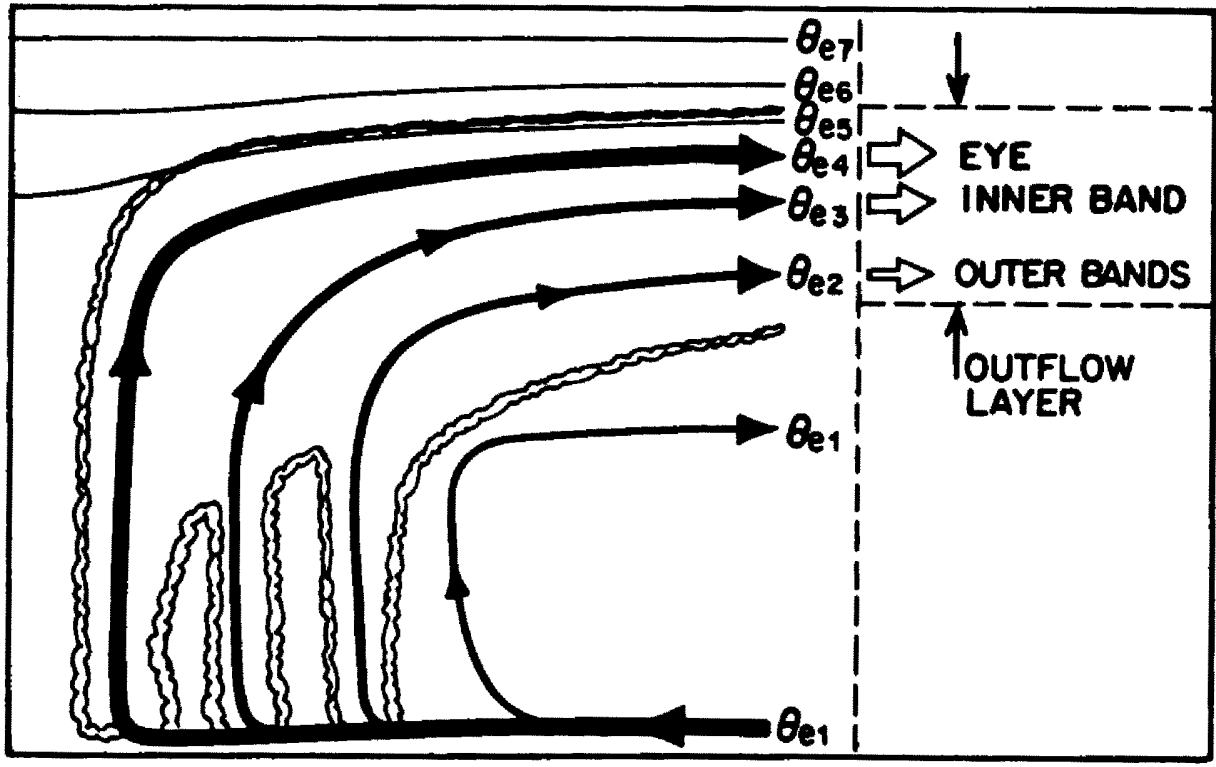

F1g. 5.2. Schematic representation of the thermodynamic aspects of cyclone-environment interactions. The lines with arrows are $\theta$ contours which, outside the PBL, colncide with the streamines of the transverse circulation for steady flow with no mixing. Open arrows outside the hurricane dcmain are enviromental conditions which are removing heat at a speclfied $\theta_{e}$ value at the same rate as heat is being added by surface fluxes in the boundary layer.

outflow corresponding to the range of $\theta_{e}$ values produced by the hurricane. Air ascending in outer bands arrives at upper levels with a "typical" tropical $\theta_{e}$ of $340 \mathrm{~K}$, while inflowing parcels are increased by surface fluxes to $\theta_{\theta}$ of $350 \mathrm{~K}$ or more and arrive at a higher level. The outflon must be able to dispose of the air from each source. If the $\theta_{e}$ associated with eyewall alr is found only in the stratosphere where surrounding synoptic systems are weak, a steady efflux of high energy air may not be sustainable. The hurricane would then lose its ability to maintain a radial circulation through this high energy area and the surface fluxes and convection would redistribute to lower $\theta_{e}$ values, although the hurricane total of convection might well remain unchanged. 
This mechanisu is admittedly highly speculative but, to the extent that outflow constriction and concentric eye development are both related to arrested intensification, a link between them should at least be considered.

A final comment on the influence of internal hurricane structure on intensity and intensity change stems fram the work of Weatherford (1985) with Air Force reconnalssance data from typhoons. As was mentioned in Chapter 1, we have found it conceptually necessary to consider the low level wind clrculation of the hurricane as being made up of an envelope of around $300 \mathrm{~km}$ radius, surrounding a core of around $100 \mathrm{~km}$ radius containing the eye-eyewall structure and perhaps a rain band or two. The speed of the maximum wind in the core (or the central pressure, which is related) is def Ined as the "Intensity", while the average wind speed in the envelope is the "strength". Only intensity is archived in most tropical cyclone records, but with the reconnaissance data it is possible to produce strength records as well. Weatherford (1985) examined various relationships between intensity, strength, and eye character. She found that intensity and strength are not well related; typhoons with very strong envelopes could have weak maximum winds or vice versa. Similarly, intensity change and strength change are not well related. Figure 5.3 , which depicts intensity change as a function of strength, does yield an interesting result. Al though the variables are uncorrelated, the intensity change "envelope" shows a strong functional relationship, with the greatest intensity change rates occurring at intermediate strengths.

The cause of this relationship is not known, but it is interesting to speculate. The upper limit of intensity change for low-strength 


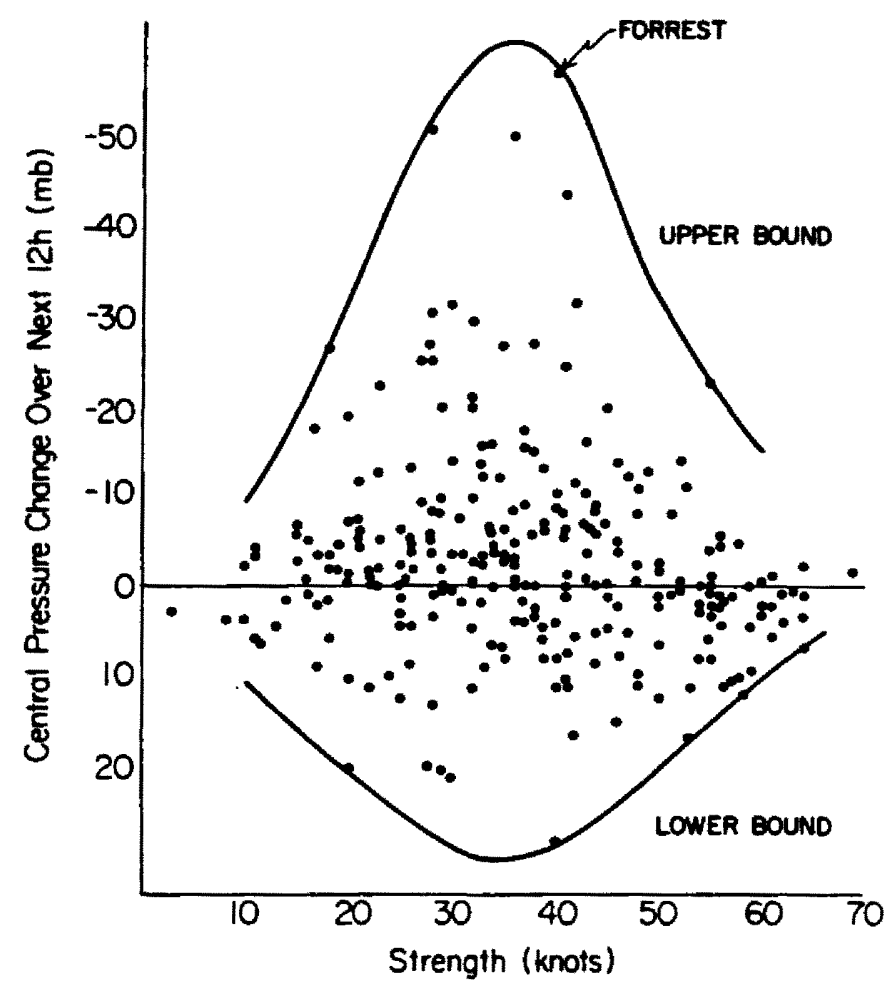

Fig. 5.3. Relationship between strength (average $700 \mathrm{mb}$ wind from $1^{\circ}$ $2.5^{\circ}$ radius) and intensity change (mb/12 h) over the following $12 \mathrm{~h}$ for Pactfic tropical cyclones, 1981-1983. Figure from Weatherford (1985).

cyclones may be imposed by the limits on the "steepness" of the wind profile. If the wind Increases inward too quickly, the anticyclonic shear $\partial v / \partial r$ would overpower the curvature $v / r$, resulting in negative vorticlty and an unstable vortex core whlch would break up. This zerovorticity flow would be associated with a tangential wind given by $v r^{x}$ = constant with $x=1$. Empirically, the upper limit is $x=0.75$ or so, while a constant stress boundary layer would lead to the commonly quoted $x=0.50$ (Malkus and Riehl, 1960). A cyclone of small strength would thus have an upper bound on intensity and 11kewise on intensity change in short periods (12 h in Fig. 5.3). Large (strong) vortices are felt to be resistant to rapid changes in intensity because of the larger area of strong winds and low pressure in the core, and the tendency for a 
larger eye. Since the pressure drop in the eye is established and maintained by the eyewall convection (Shapiro and W1lloughby, 1982), the larger eyes must be warmed over a larger area and changes might be expected to be less rapid. Other factors might be the increased vorticity and frictional convergence at radil outside the eyewall, and the larger frictional dissipation to be overcome.

Internal-dynamic problems will no doubt continue to receive a large share of the research effort, both in terms of aircraft observations and numerical modeling. Some of the questions raised in the above subsection might be clarified by the following:

1) outward extension of the Atlantic flight pattern to $150 \mathrm{n} \mathrm{m} 1$ radius or more to define quantitatively the range of strengths of Atlantic hurricanes, and

2) model experiments to determine the interdependence of strength, intensity and eye character in a controlled simulation.

5.2 Environmental Influences on Hurricane Intensity Change: A Conceptual Model

The goal of this research was to improve our understanding of how the environment influences the intensity of a hurricane. This has been accomplished, but mostly by reinforcing what has al ready been said, though not always generally accepted. The "original" findings of this research are limited to the description of outflow structure from observations (Chapter 3), and the isolation by observational means of the upper tropospheric wave associated with imports of angular momentum (Chapter 4). Dynamical "understanding" of vortex-environment interactions awalts a mathematical treatment of the wave-mean flow interactions but, in the interim, the following qualitative description should be considered. 
The environmental influences on hurricane intensity change can be summarized in two quotations, from 25 years ago.

1) "The minimum pressure of the hurricane is related to the temperature of the sea surface over which 1t moves, and there is some evidence that this relationship can be expressed on a quantitative basis. The circulation features around the storm, however, may prevent the pressure within a tropical storm from reaching its potential minimum, 1.e., warm water temperatures are a necessary but not a sufficient requirement for major intensification". (Miller, 1958).

2) "Malntainence and growth of a warm core w1ll depend at least partly on the magnitude of the ventilation compared to the heat input by the primary mass circulation"'. (Simpson and Riehl, 1958).

Three "enviromental effects" are mentioned. The first statement makes mention of a "potential intensity" which depends upon sea surface temperatures; the degree to which the potential is realized depends upon circulation features (considered by Miller, 1958 to be "the presence or absence of an efficient high-level outflow mechanism") In the hurricane enviroment. The second quotation al so states the influence of vertical wind shear in the enviroment in Inhibiting intensification.

This paper has explicitly analyzed the first two and (In the discussion of the Stationary Band Complex (W1lloughby et al., 1984) in section 5.3) re-interpreted the third. It is now possible to pose a conceptual model of environmental influences on hurricane intensity change which includes all three factors.

At the heart of this model is the notion that the fully developed hurricane and its enviroment are in a quasi-equilibrium state, as 
described by Arakawa and Schubert (1974). The surface fluxes and convection are acting to maintaln a vertical structure which is nearly neutral for molst processes within the vortex against a large scale advective and vertical motion field which is attempting to destabilize 1t. If any one process is changed, the others will adjust and a new quasi-equilibrium state with different process rates and a slightly different structure attained.

This quasi-equilibrium state corresponds to the mature stage reached by a hurricane model, in which intensification stops because the core becomes statically stable (for moist processes) or nearly so and the vigor of the convection is reduced. This stabilization begins at the center (leading perhaps to "eye formation") and spreads outward. The clearest illustration is contained in Ooyama (1969). This stabilization and convective suppression is critical for a realistic simulation; without it the model hurricane intensifies without limit (Ogura, 1964).

The major physical processes involved are illustrated in Fig. 5.4. This figure does not involve the eye (that will be added next), nor is it a complete representation of the highly nonlinear and implicit system. Surface flux, convection, and outflow are linked so that changing one changes the others and leads to a different structure. Cut off the surface fluxes (or increase the ventilation) and the low levels will cool, increasing the molst static stability and decreasing the eyewall convection. Constrict the outflow by an environmental process which opposes lateral mixing (Fig. 5.5) and the warm core outside the eye will warm slightly, increasing the moist static stability and decreasing the eyewall convection. 
(a)

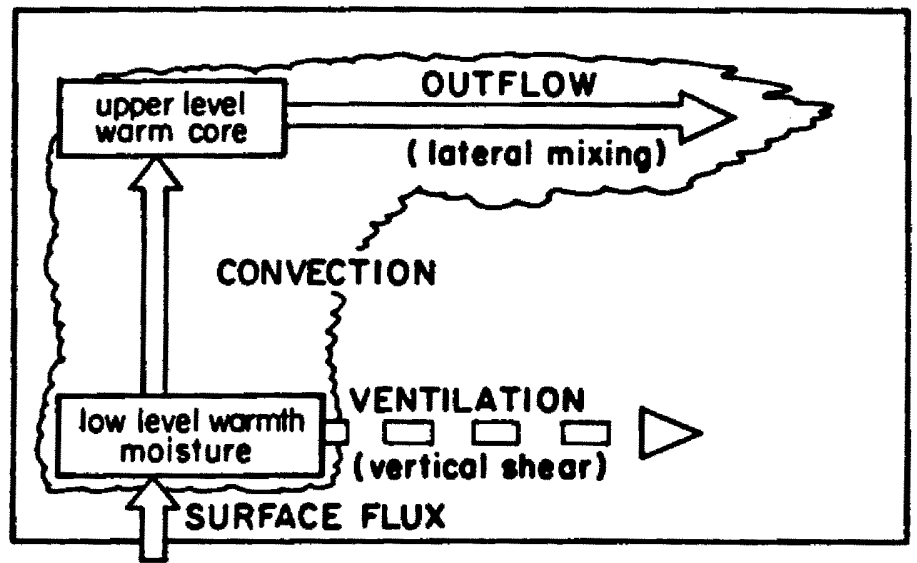

(b)

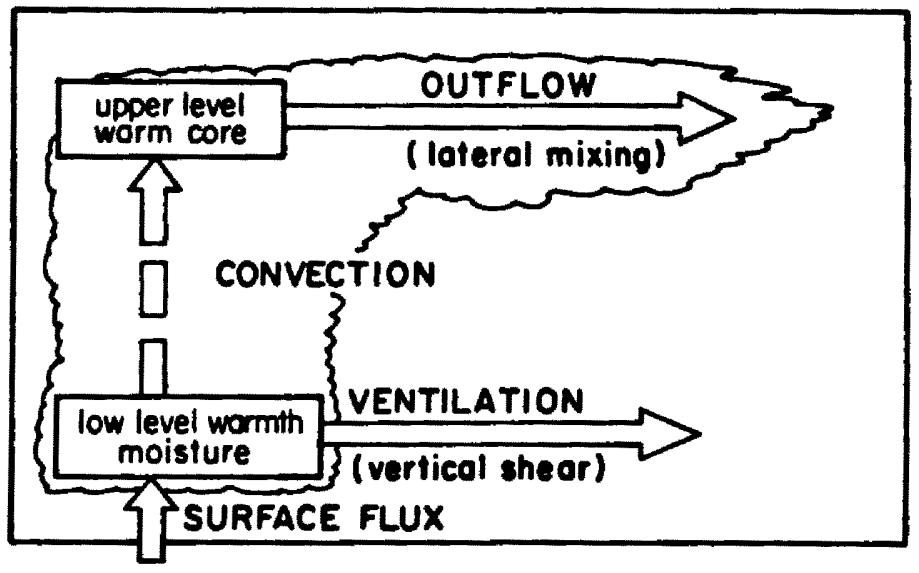

(c)

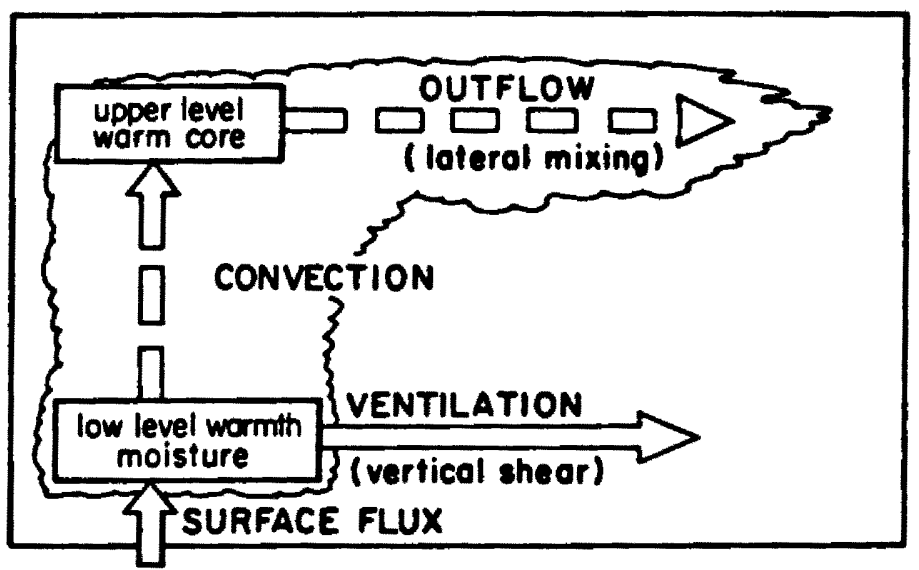

Fig. 5.4. Balance of processes in the quasi-equilibrium system of surface energy fluxes, convection, and environmental effects (outflow and ventilation) in a tropical cyclone. Three cases are shown: a) intensifying hurricane with effective outflow, minimal ventilation, and active convection, b) hurricane with increased ventilation and reduced convection, and c) hurricane with restricted outflow and reduced convection. 

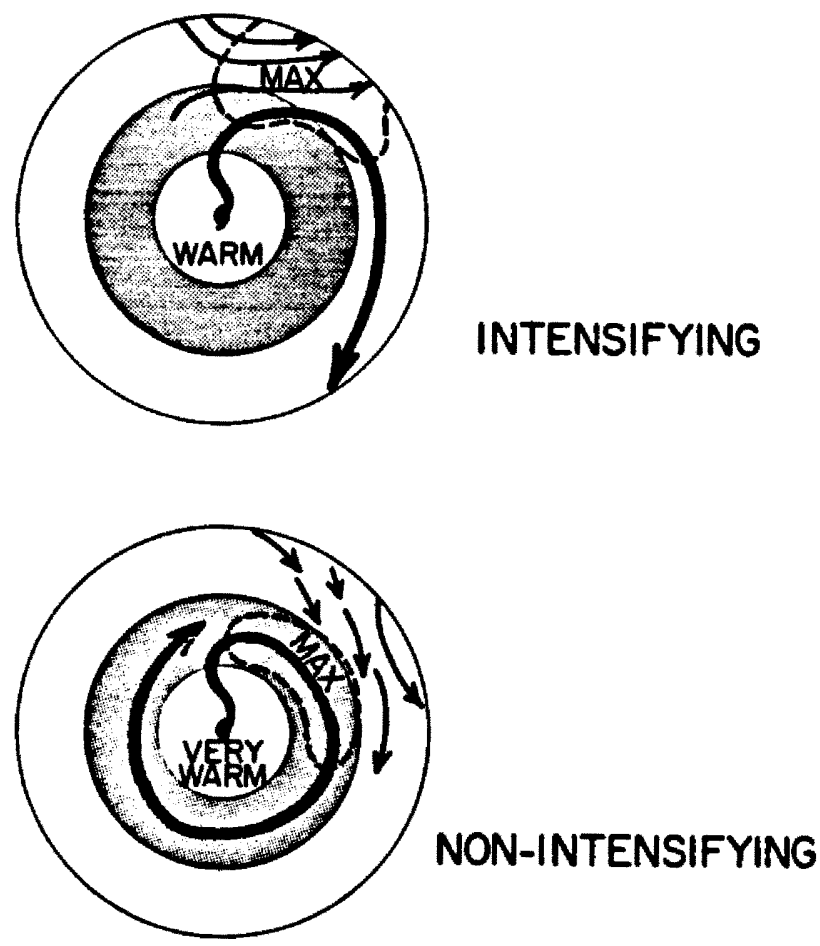

Fig. 5.5. Schematic of the upper-tropospheric interaction between a tropical cyclone and its environment. Barotropic instability within the synoptic scale warm core (Inner circle) maintains outflow in this area in both cases. Different environmental conditions (outer ring) determine whether or not the barotropic instability and lateral mixing can operate across the interface (shaded ring). If not (lower diagram), tangential wind within the interface accelerates anticyclonically and, in association, the synoptic scale warm core amplifies. Convection in the eyewall and intensification are thereby suppressed.

Direct evidence of this destabilization of the thermal

stratification was obtained after the above hypothesis was formulated. Using rawinsonde compositing methods (Frank, 1977a), two typhoon composites were prepared using 20 years of western North Pacific rawinsonde data. This data set is described in detail in Lee (1986). The typhoon positions were selected so as to have similar intensities (maximum winds of 65-125 kt) but were divided into "intensify ing" (monotonic increase in maximum winds of at least $15 \mathrm{kt}$ over the next 24 
h) and "non-1ntensifying" (maximum wind change of $10 \mathrm{kt}$ or less over the next $24 \mathrm{~h}$ and $5 \mathrm{kt}$ or less over the next $12 \mathrm{~h}$ ) categories. The difference of the azimuthal mean temperature between the two is shown in Fig. 5.6. Negative values indicate where the intensifying system is colder.

Temperatures are almost identical in the low levels near the center, reflecting the nearly homogeneous ocean temperatures in the tropical west Pacific. At outer radil (enviroment) the intensifying typhoon is slightly warmer, possibly reflecting its mean latitude of $16.5^{\circ} \mathrm{N}$, about $4^{\circ}$ equatorward of the mean nor-intensify ing typhoon. The greatest differences are in the upper levels. At outer radi1, the intensifying typhoon is colder, again possibly a reflection of its lower mean latitude and correspondingly higher and colder tropopause. But at Inner radil (less than $4^{\circ}$ latitude radius) the intensifying system is colder in the middle and upper troposphere as well, indicating a less stable stratification within the convective region of the typhoon.

This temperature difference is quite reliable. Using the temperature measurements of $200 \mathrm{mb}$ in the $1-3^{\circ}$ radius belt, a Tstatistic of 7.5 for the temperature difference was obtained. The mean typhoon characteristics for the $1-3^{\circ}$ radius belt only were recalculated to Insure that the difference was not a result of a sampling bias in the typhoon positions which contributed soundings to this core region. The statistical properties for the $1-3^{\circ}$ belt were found to be similar to those for the entire composite. The intensifying typhoon had a central pressure only $3 \mathrm{mb}$ higher than that of the non-intensifying typhoon and was still at nearly the same longitude and about $6^{\circ}$ latitude 


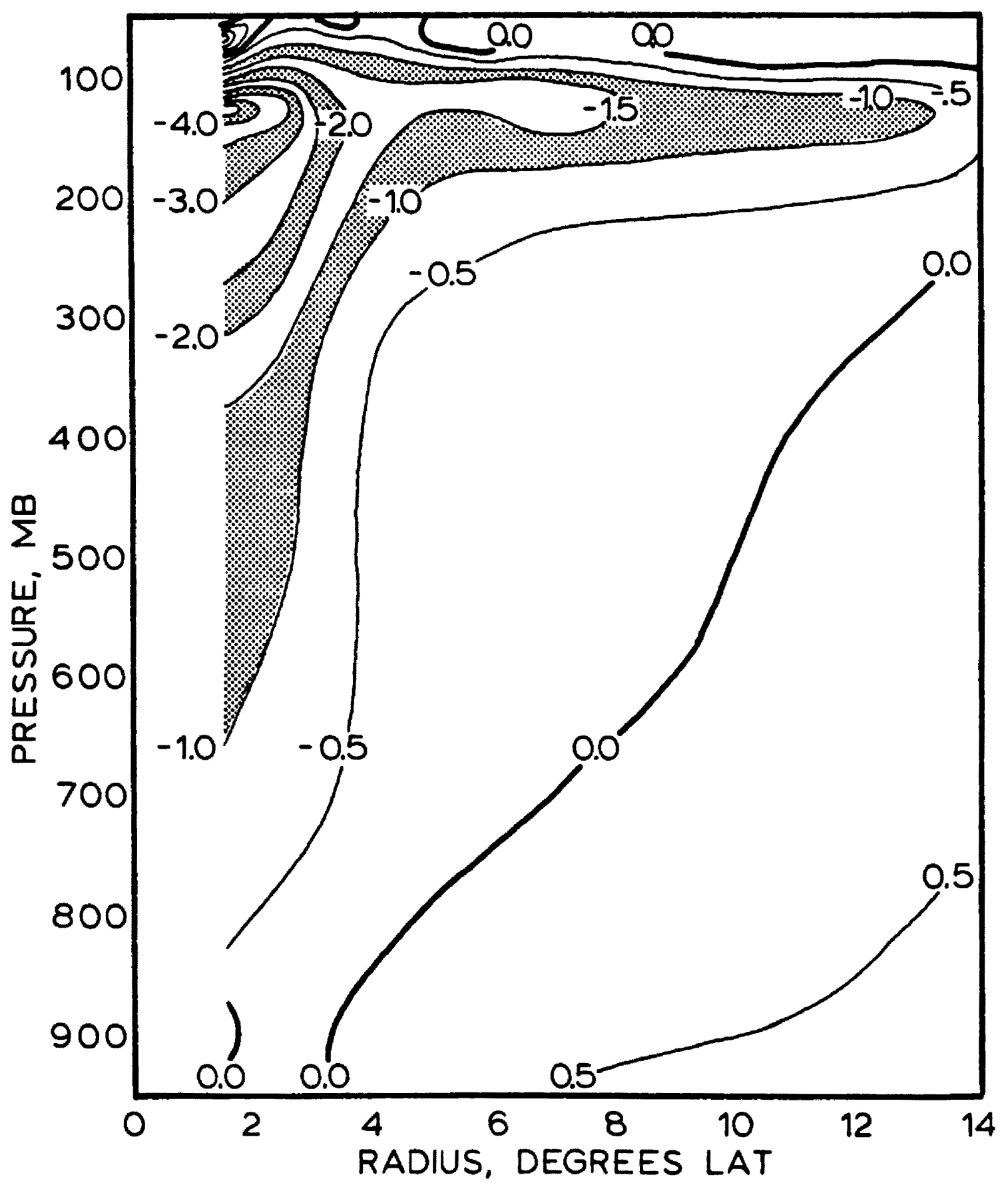

F1g. 5.6. Temperature difference between a composite intensify ing typhoon and non-intensifying typhoon of similar current intensity. Negative values indicate areas where the intensifying typhoon is cooler. See text for complete description. 
equatorward. The temperature difference therefore cannot be explained by the intensifying typhoon sample being: a) in a climatologically colder environment, or b) initially much less intense. The only difference (besides the lower static stability) is that the intensifying typhoon has lower strength $\left(700 \mathrm{mb} 1-3^{\circ}\right.$ tangential winds of $17.4 \mathrm{~m} \mathrm{~s}^{-1}$ for the intensifying typhoon and $22.1 \mathrm{~m} \mathrm{~s}^{-1}$ for the non-intensifying typhoon respectively).

The discussion to this point does not include hurricane intensity per se, only convection rate. Emplrically, the two are related. however. Satelilte measurements of cloud top temperatures in eyewalls (Arnold, 1977; Gentry et al., 1980; Dvorak, 1984) Indicate that intense or Intensifying systems are associated with cold cloud tops near the center, Indicating vigorous convection. Discussions with veterans of typhoon reconnaissance also indicate that intensifying systems have greater turbulence and more vigorous convection than steady or filling systems of equivalent intensity. The vigor of the eyewall convection is presumed to be regulated by the environmental effects of ventilation and outflow-layer interaction, with convection and intensity related by another quasi-equilibrium loop involving the eye structure. The warmth of the eye is maintained by subsidence of atr which ascended in the eyewall convection, and is opposed by mixing of eyewall air into the eye and evaporation of condense te which is mixed into the eye. The warming depends upon the rate of convective heating (which is in quasiequilibrium with the ventilation and outflow) and the area over which warming occurs (eye diameter) while the cooling is related to eye structure; mid-tropospheric shear which leads to flow asymmetries and a 
vertically tilted eye would tend to increase mixing of eyewall air and condensate Imwards.

What then of Conditional Instability of the Second Kind (CISK)? As defined by Ooyama (1982), CISK refers to the cooperative Interaction between convection and vortex which intensifies and maintains the latter, without specific reference to environmental processes which may also be influencing convection. At the heart of the CISK concept is a positive feedback between warming and disturbance amplitude. In the linear theory of Charney and Eliassen (1964), the heating and warming are proportional to the frictional convergence of molsture, which inoreases as the disturbance amplifies. The feedback is between disturbance amplitude and heating, and the growth rate (warming) is proportional to the latter. Nonlinear theory (Schubert and Hack, 1982) considers a flxed heating rate, but an "efficiency" which goes up with Inoreasing inertial stability so that the warming (and deepening rate) is a function of disturbance amplitude. The feedback is between disturbance amplitude and warming (growth rate), with heating fixed. What happens in nature (and also in models) is that with time, disturbance amplification and the associated upper-level warming begin to inhibit the buoyant convection and heating by increasing the static stability as discussed earlier resulting in a negative feedback. The first manifestation of this is eye formation, which removes heating from the most "efficlent" part of the vortex (h1ghest inertial stability) and slows the growth rate (Schubert and Hack, 1982). By combining this consideration with the nonlinear "CISK" of the previous paragraph, one can arrive at FIg. 5.7 , which shows how a heating rate and warming 


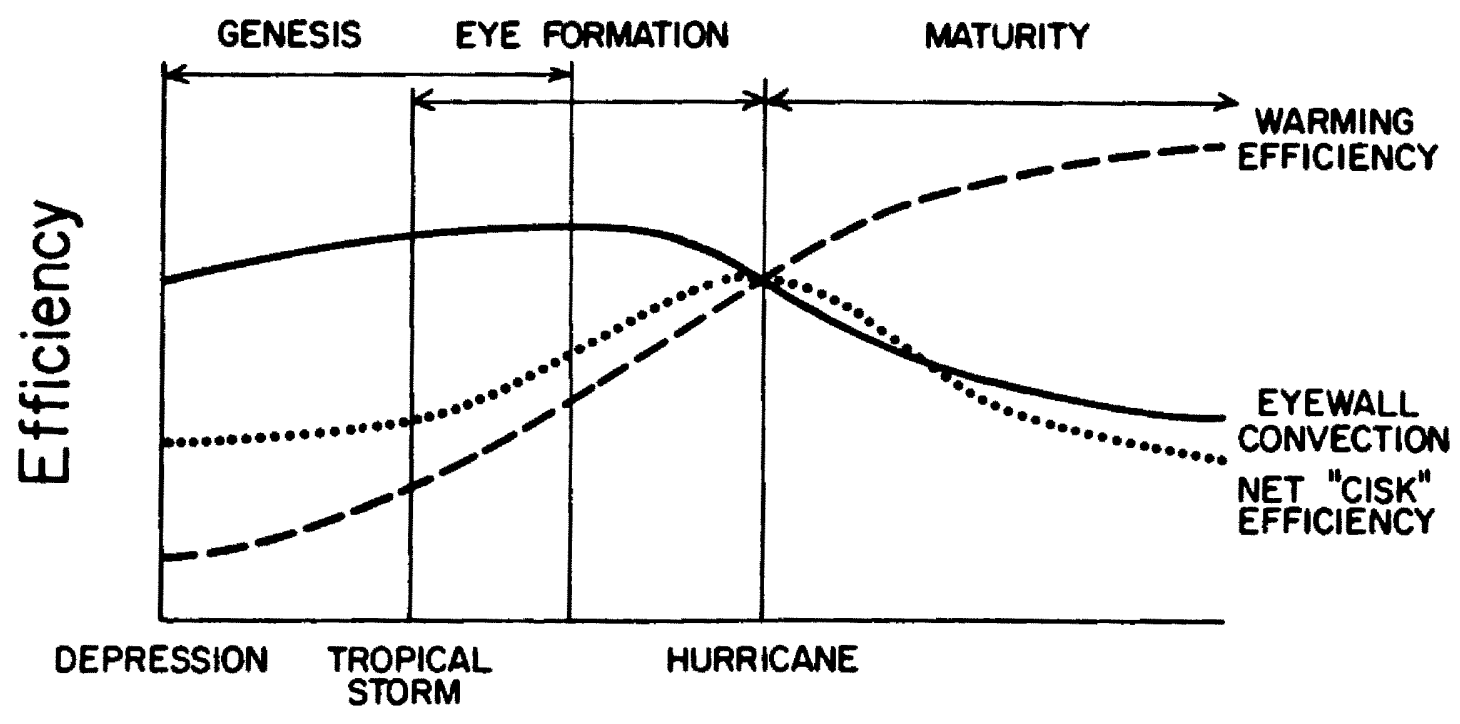

\section{Vortex Stage}

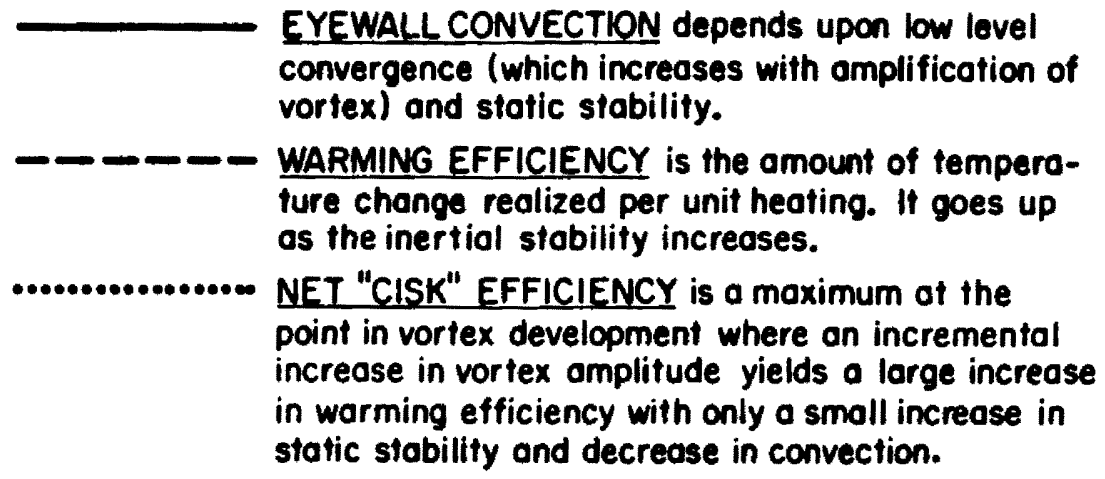

Fig. 5.7. Synthesis of CISK concepts as discussed by Charney and Eliassen (1964) and Schubert and Hack (1982). See text for discussion. 
efficiency might combine to produce an internal stability parameter and define the range of vortex states over which CISK operates.

CISK can be defined as occurring when an amplification of the vortex increases the growth rate such that the development can "pay its way" against increased frictional dissipation. Linear CISK is described by the solid line during the "genesis" stage, when increasing the vortex amplitude causes more frictional convergence and heating. As the development proceeds towards the right, the increasing static stability and heating reduction (not considered in linear theory) eventually results in a negative feedback between eyewall convection and the vortex amplitude. Nonlinear CISK (Schubert and Hack, 1982; dashed line) operates over all ranges of vortex development, but (mathematically though not conceptually) neglects convective suppression. Combining the two CISKs (dotted line) yields a "CISK" feedback which operates with greatest effectiveness up until eye formation. The hurricane life cycle can thus be broken into three phases. In "genesis"'and "maturity," heating rate and net ef ficiency change relatively little for a change in vortex stage, and the quasiequilibrium state between environment and vortex is most apparent. In the Intermediate stage leading up to eye formation, internal parameters are changing rapidly and a "CISK" process is significant, if not dominant, in determining the growth rate of the vortex.

\subsection{A Research Agenda}

Principal results of this paper have been:

1) development of a method for studying the synoptic-scale asymmetric outflow of tropical cyclones, 
2) documentation of the typical synoptic-scale outflow patterns of Atlantic hurricanes,

3) description of some of the differences in outflow layer flow between intensifying and non-intensifying hurricanes,

4) clarification of the role of sea-surface temperature in defining an upper bound on hurricane intensity,

5) depiction of the upper tropospheric flow asymmetry responsible for synoptic-scale eddy imports of relative angular momentum into hurricanes and of the existence of a barotropically unstable basic state belleved to be responsible for this asymmetry, and

6) observation of static stability reductions believed to be associated with enhanced convective activity in intensifying typhoons.

Section 5.2 attempts to combine these findings into a conceptual model of environmental flow (outflow structure and vertical shear) effects on the intensity of hurricanes through modification of the static stability and convective activity in the eyewall region. This model hints at several processes which are believed to be occurring but which are not documented or understood:

1) a wave-mean flow interaction at radil of $500 \mathrm{~km}$ or less which is acting to reduce the vertical shear of the tangential wind from middle to upper troposphere by the convergence of relative angular momentum above $300 \mathrm{mb}$.

2) a response of the symmetric flow to (1) in the form of an adjustment process which tends to cool the upper troposphere over the eyewall and inner convective bands, 
3) a wave-wave interaction (between an asymmetric enviroment and the wave alluded to in 1 ) at radil of $1500 \mathrm{~km}$ and beyond which modulates the effect described in 1) and therefore the temperature structure, and

4) a convective response by the eyewall which results in an acceleration of the secondary circulation in the eye-eyewall system and a reduction in surface pressure and/or increase in low-level maximum sustained winds.

Additional observational studies and a beginning of theoretical work are essential if we are to learn if, and how, these processes work. Specific suggestions are outlined below.

Observational studies. Observational programs should seek to:

1) monitor the temporal changes in upper tropospheric thermal structure of the eye-eyewall system and the convective activity (out to a radius of $200-300 \mathrm{~km}$ ) to document the existence of a relationship between static stability, convective activity, and intensity change, especially in symmetric hurricanes in weakly sheared environments,

2) monitor the temporal changes in upper tropospheric wind structure at inner radii (of $300 \mathrm{~km}$ or less) especially the magnitude of eddy angular momentum flux convergence which might be causing changes in the axisymmetric temperature structure through an adjustment process,

3) monitor the temporal changes in upper tropospheric wind structure at outer radil to diagnose the wave-wave interactions between the unstable modes identified by modeling studies (described below) and the enviromental flow, and

4) measure the vertical structure of the outflow layer from $300 \mathrm{mb}$ to $100 \mathrm{mb}$ over as large a radial domain as is possible, in order to determine the disposition of alr having low (outer convection) and high 
(eyewall) $\theta_{e}$ values as a first step towards testing the concept illustrated in Fig. 5.2.

Data sources and methodology will depend upon the particular problem. Item 1) is amenable to satellite remote sensing, and 3) can be pursued using the combined wind set and upgraded processing as described at the end of Chapter 2. Item 2) may be possible using high time resolution satellite imagery to derive winds within the hurricane's cirrus shield, but will be better suited to study by research aircraft. Item 4) will almost certainly require an upper-tropospheric research flying program, which would also be of great help in pursuing item 1 ). Research to study 1tems 2) and 3) should probably be begun only after the modeling of upper-tropospheric wave activity (described next) is well underway, so that a list of specific quantities to be measured or calculated is in hand.

Hodeling. The suggestions for modeling studies are aimed at using existing technology wherever possible. Suggested modeling problems are:

1) Isolation of the natural instabilities of an axisymmetric basic state flow on a f-plane representative of a mature hurricane over radii out to at least $1500 \mathrm{~km}$. Particular attention should be paid to modes which act to reduce the magnitude of the warm anomaly of the upper tropospheric structure from the radius of maximum wind out to around 300 $\mathrm{km}$, as waves which accomplish this may be associated with static stability alterations intensity modulation.

2) Solution for the linear wave structure supported by the basic state in 1) but with a specifled tangential wavenumber and amplitude as an outer boundary condition, corresponding to an "environmental effect" (Stevens, 1985). A range of "envirormental effects" should be 
used to identify that which produces the greatest rate of reduction of temperature as described in (1).

The above can be accomplished with a linear model. The next level of refinement is to include nonlinear effects so that the waves can alter the basic state and can affect each other. Addition of friction, molsture, and cumulus parameterization (in terms of the axisymmetric basic state flow) would allow the simulation of the enviromenthurricane quasi-equilibrium conditions by allowing model vortices to develop until they approach steady state, but with different specified outer boundary conditions. Different steady state intensities should be obtained for different enviromental conditions. Sinilar experiments, but with specified wavemean flow interactions instead of just specified outer boundary conditions were performed by Challa and Pfeffer (1980). The use of a specified but time-varying "enviroment" might al so be used to simulate intensity change of mature hurricanes, and varying the helght at which the environmental forcing is applied could be done to attempt to drive a concentric eye cycle as per Fig. 5.2.

\subsection{Operational Implications}

This paper began with some examples of how rapidly hurricanes can increase in destructive power, and the author's primary motivation for attacking this difficult subject was the threat posed by rapid intensification to our ever more populous coasts. Forecasters have long used qualitative rules of thumb based on the 200 mb flow to predict intensification without really understanding the processes at work. It is hoped that the compositing results of Chapter 3 and the conceptual model of this chapter will ald in the qualitative use of $200 \mathrm{mb}$ maps. 
Figure 3.24 shows the areas of the outflow pattern where differences between intensifying and non-intensifying cyclones were noted. It is hoped that further work will allow these characteristics to be quantified and perhaps incorporated into a statistical technique along with sea surface temperature, eye diameter, and perhaps cloud-top temperature information. The feasibility of numerical techniques for intensity change prediction will depend upon the outcome of numerical experiments outlined in section 5.3 . 


\section{ACKNOWLEDGEMENTS}

I am very thankful to have had the opportunity to study this topic which has been of longstanding personal interest. My advisor, Professor William M. Gray, provided a mixture of encouragement, freedom, and criticism which proved invaluable. The ideas developed in this paper stem from many conversations with Dr. Greg Holland and Professor Wayne Schubert about tropical cyclones, and discussions with Cheng-Shang Lee, Roger Edson, and Professor Duane Stevens helped bring these ideas to frustion. The combined wind data set was assembled with the help of Mark Zimmer and Charlie Neumann of the National Hurricane Center and Bill Thorson of Colorado State University. Drafting by Cindy Schrandt and typing by Barbara Brumit and Patti Nimmo made the transition from thoughts to paper possible.

This material is based upon work supported jolntly by the National Science Foundation and the National Oceanic and Atmospheric Administration under Grant Number ATM 8419116.

And special thanks to my parents and Paul and Terry Clesielski for their help during my less happy days. 


\section{REFERENCES}

Akima, Y., 1970: A new method of interpolation and smooth curve fitting based on local procedures. I. Assoc. Comput. Mach, 17, 589-602.

Alaka, M. A., 1961: The occurrence of anomalous winds and their

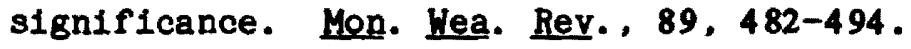

Alaka, M. A., 1962: On the occurrence of dynamic Instability in inciplent and developing hurricanes. Mon. Wea. Rev., 90, 49-58.

Alaka, M. A., 1963: Instability aspects of hurricane genesis. National Hurricane Research Project Rep. 64, 23 pp.

Anthes, R. A., 1972: The development of asymmetries in a threedimensional numerical model of the tropical cyclone. Mon. Hea. Rey. , $100,461-476$.

Anthes, R. A., 1982: Tropical cyclones, thelr evolution, structure and effects. Meteor. Monographs, Vol. 19, AMS, 45 Beacon St., Boston, MA, 02108, 208 pp.

Anthes, R. A., and S. W. Chang, 1978: Response of the hurricane boundary layer to changes of sea-surface temperature in a numerical model. J. Atmos. Sc1. , 35, 1240-1255.

Arakawa, H., 1950: Mame-taifu or midget typhoon. Geophys. Mag., 23, $463-474$.

Arakawa, A. and W. H. Schubert, 1974: Interaction of a cumulus cloud ensemble with the large-scale environment, Part I. J. Atmos. Sc1. , 31, 674-701.

Arnold, C. P., 1977: Troplcal cyclone cloud and intensity relationships. Dept. of Atmos. Sc1. Paper No. 277, Colo. State Univ., Ft. Collins, CO, 154 pp.

Bauer, K. G., 1976: A comparison of cloud motion winds with coinciding radiosonde winds. Kon. Hea. Rev., 104, 922-931.

Black, P. G. and R. A. Anthes, 1971: On the asymmetric structure of the tropical cyclone outflow layer. J. Atmos. Sc1. , 28, 1348-1366.

Brand, S., 1970: Geographic and monthly variation of very large and very small typhoons of the western North Pacific Ocean.

NAVWEARSCHFAC Technical Paper 13-70, 27 pp. [Avallable from Naval Environmental Prediction Research Facility, Monterey, CA, 93940$].$ 
Brand, S., 1972: Very large and very small typhoons of the western North Pacific Ocean. J. Meteor. Soc. of Japan, 50, 332-341.

Brand, S., and C. P. Guard, 1979: An operational study of extratropical storms evolved from tropical cyclones in the western North Pacific. I. Meteor. Soc. of Japan, 57, 479-483.

Boyd, J. P., 1976: The noninteraction of waves with the zonally averaged flow on a spherical earth and the interrelationship of eddy fluxes of energy, heat, and momentum. I. Atmos. Sci., 33, 2285-2291.

Carlson, T. N., 1967: Structure of a steady state cold low. Mon. Hea. Rev., 95, 763-777.

Carter, T. M., 1985: A general strategy for hurricane evacuation decision making. Seminar presented at Cooperative Institute for Research in the Atmosphere (CIRA), Colo. State Univ., Ft. Collins, Co, July, 1985 .

Case, R. A., and H. P. Gerrish, 1984: Atlantic hurricane season of 1983. Mon. Hea. Rey., 112, 1083-1092.

Charney, J. G., 1947: The dynamics long waves in a baroclinic westerly current. J. Meteor., 4, 135-162.

Charney, J. G., and P. G. Drazin, 1961: Propagation of planetary-scale disturbances from the lower into the upper atmosphere. $J$. Geophys. Res. . 66, 83-109.

Charney, J. G., and A. Eliassen, 1964: On the growth of the hurricane depression. I. Atmos. Sci. , 21, 68-75.

Challa, M. and R. L. Pfeffer, 1980: Effects of eddy fluxes of angular momentum and model hurricane development. J. Atmos. Sci., 37. 1603-1618.

Chen, L. and W. M. Gray, 1984: Global view of the upper level outflow patterns associated with tropical cyclone intensity changes during FGGE. Postprints, 15 th Technical Conference on Hurricanes and Iropical Meteorology, AMS, January 9-13, Miam1, FL, 224-231.

Chen, L. and W. M. Gray, 1985: Global view of the upper level outflow patterns associated with tropical cyclone intensity changes during FGGE. Colo. State Univ. Atmos, Sci. Paper 392, 126 pp.

Clark, G. B., 1983: Atlantic hurricane season of 1982: Kon. Wea. Rey., 111, 1071-1079.

Cline, I. M., 1926: Tropleal Cyclones. McGraw Hill Book Co., New York, 301 pp.

Colon, J. A., 1964: On the structure of Hurricane Helene (1958). NHRP Rept. No. $72,56 \mathrm{pp}$. 
Colon, J. A., and Staff, 1961: On the structure of Hurricane Da1sy (1958). National Hurricane Research Project Paper No. 48, 102 pp.

Cressman, G. P., 1959: An operative objective analysis scheme. Mon. Nea. Rey., 87, 367-374.

Dropco, K. M., 1981: Tropical cyclone intensity change - a quantitative forecasting scheme. Dept. of Atmos. Sc1. Paper No, 333, Colo. State Univ., Ft. Collins, CO, 89 pp.

Dunn, G. E., 1951: Tropical cyclones. Compendium of Meteorology, American Meteor. Soc., 45 Beacon St., Boston, MA, 02108, 887-901.

Dunn, G. E., W. R. Davis, and P. L. Moore, 1955: Hurricanes of 1955. Mon. Hea. Rev., 83, 315-326.

Dunn, G. E., and B. I. Miller, 1964: Atlantic Hurricanes. Louisiana State Univ. Press, $326 \mathrm{pp}$.

Dvorak, V. F., 1984: Tropical cyclone intensity analysis using satellite data. NOAA Tech. Rept. NES015 11, 47 pp.

Edmon, H. J., B. J. Hoskins, and M. E. McIntyre, 1980 : Eliassen-Palm cross sections for the troposphere. J. Atmos. Sci., 37, 26002616.

Edson, R., 1986: Forthcoming paper on upper tropospheric and lower stratospheric structure of tropical cyclones. Dept. of Atmos. Sci., Colo. State Univ.

Eliassen, A., 1951: Slow thermally or frictionally controlled motions in a circular vortex. Astrophys. Nory., 5, 19-60.

Eliassen, A., 1971: On the Ekman layer in a circular vortex. J. Meteor. Soc. of Japan, 49, 784-789.

Eliassen, A., and E. Palm, 1961: On the transfer of energy in stationary mountain waves. Geophys. Publ., 22, No. 3, 1-23.

Emanuel, K. A., 1985: An alr-sea interaction theory for tropical cyclones: Part I. Unpublished manuscript, $75 \mathrm{pp}$.

Frank, W. M., 1977a: The structure and energetics of the tropical cyclone, I: Storm structure. Mon. Wea. Rey., 105, 1119-1135.

Frank, W. M., 1977b: The structure and energetics of the tropical cyclone, II: Dynamics and energetics. Kon. Hea. Rey., 105, 1136-1150.

Gentry, R. C., 1969: Project STORMFURY. Bull. Amer. Keteor. Soc., 50, 404-409. 
Gentry, R. C., E. Rodgers, J. Steranka, and W. Shenk, 1980: Predicting tropical cyclone intensity using satellite measured equivalent blackbody temperature of cloud tops. Mon. Hea. Rev., 108, 445455 .

Gorshkov, S. G., 1979: Atlas of the oceans, Yol, 2: Atlantlc and Indian Oceans. Pergamon Press, Maxwell House, Falrview Park, Elmsford, NY, 10523, 306 pp.

Gray, W. M., 1968: Global view of the origin of troplcal disturbances and storms. Mon. Yea. Rey., 96, 669-700.

Gray, W. M., 1979: Hurricanes: their formation, structure, and likely role in the tropical cyclone. Meteorology over the tropical

oceans. D. B. Shaw, Editor, Roy. Meteor. Soc., 155-218.

Gray, W. M., 1984: Atlantic seasonal hurricane frequency: Part I: El Nino and $30 \mathrm{mb}$ quasi-biennial oscillation influences. Mon. Hea. Rey., 112, 1649-1668.

Gray, W. M. and D. J. Shea, 1973: The hurricane's inner core region, II: Thermal stability and dynamic characteristics. J. Atmos. Sci. , 8, 1565-1576.

Haurwitz, B., 1935: The helght of troplcal cyclones and the eye of the storm. Mon. Hea. Rey. , 63, 45-49.

Hawkins, H. F., and S. M. Imbembo, 1976: The structure of a small intense hurricane - Inez 1966. Mon. Wea. Rey., 104, 418-442.

HawkIns, H. F., and D. T. Rubsam, 1968a: Hurricane Hilda, 1964. I. Genesis, as revealed by satellite photographs conventional and airoraft data. Mon. Hea. Rev., 96, 428-452.

Hebert, P. J., 1978: Intensification criteria for tropical depressions in the western North Atlantic. Mon. Wea. Rev., 106, 831-840.

Hebert, P. J., and B. R. Jarvinen, 1977: National Hurricane Center (NHC) diagnostic calculations on tropical cyclones. Papers, 11th Technical Conf, on Hurricanes and Tropical Meteorology, Miami Beach, FL, Dec. 13-16, 1977, 247-252.

Hebert, P. J., and K. O. Poteat, 1975: A satellite classification technique for subtropical cyclones. NOAA Tech. Meme. NWS SR-83, 25 pp.

Holland, G. J., 1983: Angular momentum transports in tropical cyclones. Quart. I. Roy. Meteor. Soc. , 109, 187-209.

Holland, G. J., and R. T. Merrill, 1984: On the dynamics of tropical cyclone structural changes. Quart. J. Roy. Met. Soc., 110, 723745 . 
Holliday, C. R. and A. H. Thompson, 1979: Climatological characteristics of rapidly intensifying typhoons. Kon. Nea. Rey.. $107,1022-1034$.

Hughes, R., 1966: In Hazard. Time-Life Books, 229 pp.

Jarvinen, B. R., and E. L. Caso, 1978: A tropical cyclone data tape for the North Atlantic basin, 1886-1977: contents, limitations, and uses. NOAA Tech. Memo. NHS NHC-6, $19 \mathrm{pp}$.

Jones, R. W., 1977: A nested grid for a three-dimensional model of a tropical cyclone. I. Atmos. Sc1., 34, 1528-1553.

Jordan, C. L., 1952: On the low-level structure of the typhoon eye. J. Meteor.. 9, 285-290.

Jordan, C. L., 1961: Marked changes in the characteristics of the eye of intense typhoons between the deepening and filling stages. $\mathrm{J}$. Neteor., 15, 779-789.

Jorgensen, D. P., 1984: Mesoscale and convective scale characteristics of mature hurricanes. Part I: General observations by research aircraft. I. Atmos. Sci., 41, 1268-1285.

Kelley, W. E., and D. R. Mock, 1982: A diagnostlc study of uppertropospheric cold lows over the western North Pacific. Nop. Wea. Rey., 110, 471-480.

Kuo, H. L., 1959: Dynamics of convective vortices and eye formation. The Atmosphere and Sea in Motion. Rossby Memorial Volume, B. Bol In, Ed., Rockefeller Institute Press, 413-424.

Kurihara, Y., 1976: On the development of spiral bands in a tropical cyclone. J. Atmos. Sc1., 33, 940-958.

Kurihara, Y., and R. E. Tuleya, 1974: Structure of a tropical cyclone developed in a three-dimensional numerical simulation model. I. Atmos. Sc1., 31, 893-9191.

Lawrence, M. B. , and J. M. Pelissier, 1981 : Atlantic hurricane season of 1980. Yon. Hea. Rey., 109, 1567-1582.

Lee, C. S., 1986: Forthcoming Dept. of Atmos. Sc1. Ph.D. Thesis on tropical cyclogenesis. Colo. State Univ., Ft. Collins, C0, 80523.

Lewis, B. M., and D. P. Jorgensen, 1978: Study of the dissipation of Hurricane Gertrude (1974). Mon. Wea. Rey., 106, 1288-1306.

L1lly, D. K., and K. A. Emanuel, 1985: A steady-state hurricane model. Extended Abstracts Volume, 16th Conf, on Hurrtcanes and Troplcal Meteorology, Amer. Meteor. Soc., 45 Beacon St., Boston, MA, 02108 , 144-145. 
Lord, S. J., H. E. Willoughby, and J. M. Piotrow1cz, 1984: Role of a parameterized 1ce-phase microphysics in an axisymmetric, nonhydrostatic tropical cyclone model. J. Atmos. Sci. , 41, 28362848 .

Malkus, J. S., 1958: On the structure and maintainence of the mature hurricane eye. ㄱ. Meteor. , 15, 337-349.

Malkus, J. S., and H. Riehl, 1960: On the dynamics and energy transformations in steady-state hurricanes. Tellus, 12, 1-20.

Marks, F. D., 1985: Evolution of the structure of precipitation in Hurricane Allen (1980). Mon. Hea. Rey.. 113, 909-930.

Mathur, M. B., 1974: A multiple grid primitive equation model to simulate the development of an asymmetric hurricane (Isbell, 1964). J. Atmes. Sc1., 31, 371-393.

Mathur, M. B., 1975: Development of banded structure in a numerically simul ated hurricane. I. Atmos. Sc1., 32, 512-522.

McBride, J. L., 1981: Observational analysis of tropical cyclone formation. Part III: Budget analysis. I. Atmos. Sci., 38 , 1152-1166.

MaBride, J. L., and R. Zehr, 1981: Observational analysis of tropical cyclone formation. Part II: Comparison of non-developing versus developing systems. I. Atmos. Sci., 38, 1132-1151.

McDonald. N. J., 1968: The evidence for the exlstence of Rossby-like waves in the hurricane vortex. Tellus, 20, 138-150.

McDonald, W. F., 1935a: The hurricane of August 31 to September 6 , 1935. Hon. Hea. Rey., 63, 269-271.

McDonald, W. F., 1935b: Lowest barometer reading in the Florida Keys storm of September 2, 1935. Kon. Nea. Rev., 63, 295.

Merrill, R. T., 1982: A comparison of large and small tropical cyclones. Dept. of Atmos. Sc1. Paper No. 352, Colo. State Univ., Ft. Collins, Co, $75 \mathrm{pp}$.

Merrill, R. T., 1984a: Structure of the tropical cyclone outflow layer. Postprints, 15th Technical Conference on Hurricanes and Tropical Yeteorology, AMS, January 9-13, Miam1, FL, 421-426.

Merrill, R. T., 1984b: Comparisons of large and small tropical cyclones. Mon. Wea. Rev., 112, 1408-1418.

Merritt, E. S., and R. Wexler, 1967: Cirrus canopies in tropical storms. Kon. Hea. Rev., 95, 111-120. 
Miller, B. I., 1958: On the maximum intensity of hurricanes. J. Yeteor., 15, 184-195.

Miller, R. C., 1972: Notes on analys1s and severe-storm forecasting procedures of the Air Force Global Weather Central. Air Neather Service Tech. Report 200 (revised), 181 pp.

Moore, P. L., and Staff, 1957: The hurricane season of 1957. Kon. Hea. Rey., 85, 401-408.

Neumann, C. J., M. B. Lawrence, and E. L. Caso, 1977: Monte Carlo significance testing as applied to statistical tropical cyclone prediction models. I. Appl. Meteor. , 16, 1165-1174.

Nunez, E., 1981: Tropical cyclone structure and intensity change. Dept. of Atmos. Sc1. Ph.D. Thesis, Colo. State Univ., Ft. Collins, CO, $192 \mathrm{pp}$.

Ogura, Y., 1964: Frictionally controlled, thermally driven circulation in a circular vortex with application to tropical cyclones. I. Atmos. Sci. , 21, 610-621.

Coyama, K. V., 1964: A dynamical model for the study of tropical cyclone development. Geofls. Int.. 4, 187-198.

Ooyama, K. V., 1969: Numerical simulation of the life cycle of tropical cyclones. J. Atmos. Sci., 26, 3-40.

Ooyama, K. V., 1982: Conceptual evaluation of the theory and modeling of the troplcal cyclone. I. Yeteor. Soc. of Japan, 60, 369-380.

Palmen, E., 1948: On the formation and structure of the tropical hurricane. Geophysica, 3, 26-38.

Palmen, E., 1958: Vertical clrculation and release of kinetic energy during the development of Hurricane Hazel into an extratroplcal storm. Tellus, 10, 1-23.

Parks, P., 1968: The ratlroad that died at sea. Stephen Greene Press, Brattleboro, VT, 05301, 44 pp.

Perlroth, I., 1967: Hurricane behavior as related to oceanographic environmental conditions. Telius, 19, 259-268.

Pfeffer, R. L., 1958: Concerning the mechanics of hurricanes. I. Yeteor. , 113-120.

Pfeffer, R. L., and M. Challa, 1981: A numerical study of the role of eddy fluxes of momentum in the development of Atlantic hurricanes. I. Atmos. Sei. , 38, 2393-2398.

Ramage, C. S., 1959: Hurricane development. I. Meteor., 16, 227-237. 
Ramage, C. S., 1974: The typhoons of October 1970 in the South China seas: intensification, decay, and ocean interaction. I. Appl. Meteor. , 13, 739-751.

Redfield, W. C., 1831: Remarks on the prevalling storms of the Atlantic coast of the North American states. Amer. J. Sc1., 20, 17-51.

Reed, R. J., D. Norquist and E. E. Recker, 1977: The structure and properties of African wave disturbances as observed during Phase III of GATE. Mon. Hea. Rev., 105, 317-333.

Reed, R. J. and E. E. Recker, 1971: Structure and properties of synoptic-scale wave disturbances in the equatorial western Pacific. J. Atmos. Sct., 28, 1117-1133.

Reed, R. J. and R. H. Johnson, 1974: The vorticlty budget of synoptic scale wave disturbances in the tropical western Pacific. I. Atmos. Sc1., 31, 1784-1790.

Riehl, H., 1948: On the formation of typhoons. J. Meteor., 5, 247-264.

Riehl, H., 1951: Aerology of tropical storms. Compendium of Meteorology, Amer. Meteor. Soc., 45 Beacon St., Boston, MA, 02108.

Riehl, H., 1954: Troplcal Meteorology. McGraw Hill, New York, NY, 392 pp.

Riehl, H., 1963: Some relations between wind and thermal structure of steady state hurricanes. Nat. Hurricane Research Proj. Rept. 63. 27 pp.

Riehl, H., 1972: Intensity of recurved typhoons. J. ADDl. Meteor., 11, 613-615.

Riehl, H., 1979: Climate and Weather of the Tropics. Academic Press, 111 Fifth Ave., New York, NY, 10003, 611 pp.

Rodgers, E. B., and R. C. Gentry, 1983 : Monitoring tropical-cyclone intensity using environmental wind fields derived from shortinterval satellite images. Hon. Hea. Rev., 111, 976-996.

Rodgers, E. B., R. C. Gentry, W. Shenk, and V. Oliver, 1979: The benefits of using short-interval satellite images to derive winds for tropical cyclones. Non. Hea. Rey., 107, 575-584.

Sadler, J. C., 1978: Mid-season typhoon development and intensity changes and the tropical upper tropospheric trough. Mon. Wea. Bey., 106, 1137-1152.

Sanders, F., and J. R. Gyakum, 1980: Synoptic-dynamic climatology of the ' 'bomb"'. Mon. Hea. Rey., 108, 1589-1606. 
Sawyer, J. S., 1947: Notes on the theory of tropical cyclones. Quart. I. Rey. Yeteor. Soc., 73, 101-126.

Schubert, W. H. and J. J. Hack, 1982: Inertial stability and tropical cyclone development. I. Atmos. Sci., 39, 1687-1697.

Schubert, W. H. and J. J. Hack, 1983 : Transformed Eliassen balanced vortex model. J. Atmos. Sci., 40, 1571-1583.

Schubert, W. H., 1985: Wave mean-flow interactions and hurricane development. Extended Abstracts Volume, 16th Technical Conf, on Hurricanes and Tropical Yeteorology, May 14-17, Houston, TX, Amer. Meteor. Soc., 45 Beacon St., Boston, MA, 02108, 140-141.

Schoeberl, M. R., and R. S. Lindzen, 1984: A numerical simulation of barotropic instability. Part I: Wave-mean flow interaction. I. Atmos. Sci., 41, 1368-1379.

Shapiro, L. J., and C. J. Neumann, 1984: On the orientation of grid systems for the statistical prediction of tropical cyclone motion. Mon. Hea. Bey. , 112, 188-199.

Shapiro, L. J. and H. E. Willoughby, 1982: The response of balanced hurricanes to local sources of heat and momentum. I. Atmos. Sci. , 39, 378-394.

Shea, D. J., and W. M. Gray, 1973: The hurricane's inner core region. I. Symmetric and asymmetric structure. I. Atmos. Sei., 30 , 1544-1564.

Simpson, R. H., 1971: The decision process in hurricane forecasting. NOAA Tech. Keme. NWS SR-53, 35 pp.

Simpson, R. H., and J. S. Malkus, 1964: Experiments in hurricane modification. Sci. Amer., 211, 27-37.

Simpson, R. H., and J. M. Pelissier, 1971: Atlantic hurricane season of 1970. Hon. Hea. Rey., 99, 269-277.

Simpson, R. H., and H. Riehl, 1958: Mid-tropospheric ventilation as a constraint on hurricane development and maintainenance. Amer. Meteor. Soc. Tech. Conf. on Hurricanes, Miami Beach, FL, Nov, 1922,1958 .

Stevens, D. E., 1984: Personal communication.

Stevens, D. E., 1985: Personal communication.

Sundqvist, H., 1970: Numerical simulation of the development of tropical cyclones with a ten-level model, I. Tellus, 22, 360-390.

Sundqvist, H., 1972: Mean tropical storm behavior in experiments related to modification attempts. Tellus, 24, 6-12. 
Tul eya, R. E., and Y. Kurlhara, 1984: The formation of common vortices in a tropical numerical simulation model. Postprints, 15th Techntcal Conference on Hurricanes and Troplcal Meteorology, AMS, January 9-13, MIam1, FL, 320-324.

Weatherford, C. L., 1985: Typhoon structural varlabllity. Dept, of Atmos. Sc1. Paper 391, Colo. State Univ., Ft. Collins, C0, 80523, 75 pp.

Weatherford, C. L. and W. M. Gray, 1984: Relating typhoon intensity to outer $1-3^{\circ}$ radius circulation as measured by reconnalsance aircraft. Bostprints, 15th Technical Conference on Hurricanes and Iropical Meteorology, AMS, January 9-13, Miam1, FL, 238-242.

We1, D. W. and W. M. Gray, 1985: Studies on the cloud band of the tropical cyclone. Dept. of Atmos. Sci. Paper (forthcoming), Colo. State Univ. , Ft. Collins, C0, 80523.

Willett, H. C., 1955: A study of the troplcal hurricane along the At lantic and Gulf coasts of the United States. Inter-regional Insurance Conference, 116 John St., New York, NY, 63 pp.

Williams, K., 1970: Statistical analysis of trade wind cloud clusters in the western north Pacific. Dept. of Atmos. Sc1. Paper No. 161. Colo. State Univ., Ft. Collins, CO, 80 pp.

W1lliams, K. and W. M. Gray, 1973: Statistical analysis of satellite observed cloud clusters in the western Pacific. Tellus, 21, 313336.

Willoughby, H. E., 1977: Inertia-buoyancy waves in hurricanes. J. Atmos. Sa1. , 34, 1029-1039.

Willoughby, H. E., 1978a: A possible mechanism for the formation of hurricane rainbands. I. Atmos. Sct., 35, 838-848.

Willoughby, H. E., 1978b: The vertical structure of hurricane rainbands and their interaction with the mean vortex. J. Atmes. Sc1., 35 , 850-858.

Willoughby, H. E., 1979a: Excitation of spiral bands in hurricanes by interaction between the symmetric mean vortex and a shearing enviromental steering current. I. Atmos. Sci., 36, 1226-1235.

W1lloughby, H. E., 1979b: Some aspects of the dynamics in Hurricane Anita of 1977. NOAA Tech. Meme, ERL NHEML,-5, 30 pp.

Willoughby, H. E., J. A. Clos and M. G. Shoreibah, 1982: Concentric eye walls, secondary wind maxima, and the evolution of the hurricane vortex. J. Atmos. Sci., 39 395-411. 
Willoughby, H. E., F. D. Marks, and R. J. Feinberg, 1984: Stationary and moving convective bands in hurricanes. J. Atmos. Sc1., 41 , 3189-3211. 
APPENDIX A 
W. M. GRAY'S FEDERALLY SUPPORTED RESEARCH PROJECT REPORTS SINCE 1967

CSU Dept. of

Atmos. Sci. Report Ne.

104

114

116

124

Unnumbered

Unnumbered

140

161

179

182

\section{Beport Title, Author, Date, Agency Support}

The Mutual Variation of Wind, Shear and Baroclinicity in the Cumulus Convective Atmosphere of the Hurricane (69 pp.). W. M. Gray. February 1967. NSF Support.

Global View of the Origin of Tropical Disturbances and Storms (105 pp.). W. M. Gray. October 1967. NSF Support.

A Statistical Study of the Frictional Wind Veering in the Planetary Boundary Layer (57 pp.). B. Mendenhall.

December 1967. NSF and ESSA Support.

Investigation of the Importance of Cumulus Convection and Ventilation In Early Tropical Storm Development (88 pp.). R. Lopez. June 1968. ESSA Satellite Lab. Support.

Role of Angular Momentum Transports in Tropical Storm Dissipation over Tropical Oceans (46 pp.). R. F. Wachtmann. December 1968. NSF and ESSA Support.

Monthly Climatological Wind Fields Associated with Tropical Storm Genesis in the West Indies (34 pp.). J. W. Sartor. December 1968. NSF Support.

Characteristics of the Tornado Enviroment as Deduced from Proximity Soundings (55 pp.). T. G. Wills. June 1969. NOAA and NSF Support.

Statistical Analysis of Trade Wind Cloud Clusters in the Western North Paciflc ( 80 pp.). K. Williams. June 1970. ESSA Satellite Lab. Support.

A Climatology of Tropical Cyclones and Disturbances of the Western Pacific with a Suggested Theory for Their Genesis/ Maintenance (225 pp.). W. M. Gray. NAVWEARSCHFAC Tech. Paper No. 19-70. November 1970. (Avallable from US Navy, Monterey, CA). US Navy Support.

A diagnostic Study of the Planetary Boundary Layer over the Oceans (95 pp.). W. M. Gray. February 1972. Navy and NSF Support.

The Structure and Dynamics of the Hurricane's Inner Core Area (105 pp.). D. J. Shea. April 1972. NOAA and NSF Support. 
CSU Dept. of Atmos. Sc1. Report No.

188

189

190

196

200

212

219

224

225

\section{Report Iitle, Author, Date, Agency Support}

Cumulus Convection and Larger-scale Circulations, Part I: A Parametric Model of Cumulus Convection (100 pp.). R. E. Lopez. June 1972. NSF Support.

Cumulus Convection and Larger-scale Circulations, Part II: Cumulus and Meso-scale Interactions (63 pp.). R. E. Lopez. June 1972. NSF Support.

Cumulus Convection and Larger-scale Circulations, Part III: Broadscale and Meso-scale Considerations (80 pp.). W. M. Gray. July 1972. NOAA-NESS Support.

Characteristics of Carbon Black Dust as a Tropospheric Heat Source for Weather Modification (55 pp.). W. M. Frank. January 1973. NSF Support.

Feasibility of Beneficlal Hurricane Modification by Carbon Black Seeding (130 pp.). W. M. Gray. April 1973. NOAA Support.

Variability of Planetary Boundary Layer Winds (157 pp.). L. R. Hoxit. May 1973. NSF Support.

Hurricane Spawned Tornadoes (57 pp.). D. J. Novlan. May 1973. NOAA and NSF Support.

A Study of Tornado Proximity Data and an Observationally Derived Model of Tornado Genesis (101 pp.). R. Maddox. November 1973. NOAA Support.

Analysis of Satellite Observed Tropical Cloud Clusters (91 pp.). E. Ruprecht and W. M. Gray. May 1974. NOAA/ NESS Support.

Precipitation Characteristics in the Northeast Brazil Dry Region (56 pp.). R. P. L. Ramos. May 1974. NSF Support.

Weather Modification through Carbon Dust Absorption of Solar Energy (190 pp.). W. M. Gray, W. M. Frank, M. L. Corrin, and C. A. Stokes. July 1974.

Tropical Cyclone Genesis (121 pp.). W. M. Gray. March 1975. NSF Support. 
CSU Dept. of Atmos. Sc1. Report No.

241

243

257

258

259

Unnumbered

262

274

277
Report Title, Author, Date, Agency Support

Tropical Cyclone Genesis in the Western North Pacific (66 pp.). W. M. Gray. March 1975. US Navy Environmental Prediction Research Facility Report. Tech. Paper No. 16-75. (Available from the US Navy, Monterey, CA). Navy Support.

Tropical Cyclone Motion and Surrounding Parameter Relationships (105 pp.). J. E. George. December 1975. NOAA Support.

Diurnal Variation of Oceanic Deep Cumulus Convection. Paper I: Observational Evidence, Paper II: Physical Hypothesis (106 pp.). R. W. Jacobson, Jr. and W. M. Gray. February 1976. NOAA-NESS Support.

Data Summary of NOAA's Hurricanes Inner-Core Radial Leg Flight Penetrations 1957-1967, and 1969 (245 pp.). W. M. Gray and D. J. Shea. October 1976. NSF and NOAA Support.

The Structure and Energetics of the Tropical Cyclone (180 pp.). W. M. Frank. October 1976. NOAA-NHEML, NOAA-NESS and NSF Support.

Typhoon Genesis and Pre-typhoon Cloud Clusters (79 pp.). R. M. Zehr. November 1976. NSF Support.

Severe Thunderstorm Wind Gusts (81 pp.). G. W. Walters. December 1976. NSF Support.

Diurnal Variation of the Tropospheric Energy Budget (141 pp.). G. S. Foltz. November 1976. NSF Support. Comparison of Developing and Non-developing Tropical Disturbances ( 81 pp.). S. L. Erickson. July 1977. US Army Support.

Tropical Cyclone Research by Data Compositing (79 pp.). W. M. Gray and W. M. Frank. July 1977. US Navy Environmental Prediction Research Facility Report. Tech. Paper No. 77-01. (Available from the US Navy, Monterey, CA). Navy Support.

Tropical Cyclone Cloud and Intenstty Relationships (154 pp.). C. P. Arnold. November 1977. US Army and NHEML Support.

Diagnostic Analyses of the GATE A/B-scale Area at Individual Time Periods (102 pp.). W. M. Frank. November 1978. NSF Support. 
CSU Dept. of Atmos. Sc1. Report No.

Diurnal Varlability in the GATE Region ( 80 pp.). J. M. Dewart. November 1978. NSF Support.

Mass Divergence in Tropical Weather Systems, Paper I: Diurnal Variation; Paper II: Large-scale Controls on Convection (109 pp.). J. L. McBride and W. M. Gray. November 1978. NOAA-NHEM Support.

New Results of Tropical Cyclone Research from Observational Analysis (108 pp.). W. M. Gray and W. M. Frank. June 1978. US Navy Environmental Prediction Research Facility Report. Tech. Paper No. 78-01. (Available from the US Navy, Monterey, CA). Navy Support.

Convection Induced Temperature Change in GATE (128 pp.). P. G. Grube. February 1979. NSF Support.

Observational Analysis of Tropical Cyclone Formation (230 pp.). J. L. McBride. April 1979. NOAA-NHEML, NSF and NEPRF Support.

Tropical Cyclone Origin, Movement and Intensity Characterist1cs Based on Data Compositing Techniques (124 pp.). W. M. Gray. August 1979. US Navy Enviromental Prediction Research Facility Report. Tech. Paper No. CR-79-06. (Avallable from the US Navy, Monterey, CA). Navy Support.

Further Analysis of Tropical Cyclone Characteristics from Rawinsonde Compositing Techniques (129 pp.). W. M. Gray. March 1981. US Navy Environental Prediction Research Facility Report. Tech. Paper No. CR-81-02. (Available from the US Navy, Monterey, CA). Navy Support.

333 Tropical Cyclone Intensity Change - A Quantitative Forecasting Scheme. K. M. Dropco. May 1981. NOAA Support.

Recent Advances in Tropical Cyclone Research from Rawinsonde Composite Analysis (407 pp.). WMO Publication. W. M. Gray. 1981 .

The Role of the General Circulation in Tropical Cyclone Genesis (230 pp.). G. Love. April 1982. NSF Support. 
CSU Dept. of Atmos. Sci. Report Ne.

343

346

348

358

363

370

379
Report Title, Author, Date, Agency Support

Tropical Cyclone Movement and Surrounding Flow Relationships (68 pp.). J. C. L. Chan and W. M. Gray. May 1982. ONR Support.

Enviromental Ciroulations Assoclated with Tropical Cyclones Experiencing Fast, Slow and Looping Motions (273 pp.). J. Xu and W. M. Gray. May 1982. NOAA and NSF Support.

Tropical Cyclone Motion: Environmental Interaction PIus a Beta Effect (47 pp.). G. J. Holland. May 1982. ONR Support.

Tropical Cyclone and Related Meteorological Data Sets Available at CSU and Thelr Utilization (186 pp.). W. M. Gray, E. Buzzell, G. Burton and Other Project Personnel. February 1982. NSF, ONR, NOAA, and NEPRE Support.

A Comparison of Large and Small Tropical Cyclones (75 pp.). R. T. Merrill. July 1982. NOAA and NSF Support.

On the Physical Processes Responsible for Tropical Cyclone Motion (200 pp.). Johnny C. L. Chan. November 1982. NSE, NOAA/NHRL and NEPRE Support.

Tropical Cyclones in the Australian/Southwest Pacific Region (264 pp.). Greg J. Holland. March 1983. NSF, NOAA/NHRL and Australian Government Support.

Atlantic Seasonal Hurricane Frequency, Part I: El Nino and $30 \mathrm{mb}$ QBO Influences; Part II: Forecasting Its Variability (105 pp.). W. M. Gray. July 1983. NSF Support.

A Statistical Method for One- to Three-Day Tropical Cyclone Track Prediction (201 pp). Clifford R. Matsumoto. December, 1984. NSF/NOAA and NEPRF support.

Varying Structure and Intensity Change Characteristics of Four Western North Pacific Tropical Cyclones. (100 pp.). Cecilia A. Askue and W. M. Gray. October 1984. US Navy Environmental Prediction Research Facility Report No. CR 84-08. (Avallable from the US Navy, Monterey, CA). Navy Support. 
CSU Dept. of Atmos. Sc1. Report Noe

391

392

\section{Report It le, Author, Date, Agency Support}

Characteristics of North Indian Ocean Tropical Cyclone Activity. (108 pp.). Cheng-Shang Lee and W. M. Gray. December 1984. US Navy Environmental Prediction Research Facility Report No. CR 84-11. (Available from the US Navy, Monterey, CA). Navy Support.

Typhoon Structural Variability. (77 pp.). Cand1s L. Weatherford. October, 1985. NSF/NOAA Support.

Global View of the Upper Level Outflow Patterns Associated w1th Tropical Cyclone Intensity Change During FGGE.

L. Chen and W. Gray, 126 pp. NASA support.

Tropical cyclone structure and intensity change (290 pp.). Edwin Nunez. NSF Support. 


\begin{tabular}{|c|c|c|}
\hline \begin{tabular}{|l|r|} 
BIELIOGRAPHIC DATA & 1. Report No. \\
SHEET & ATS-394 \\
\end{tabular} & 2. & 3. Recipient's Accession No. \\
\hline 4. Titie and subtitle & & $\begin{array}{l}\text { 5. Report Date } \\
\text { December, } 1985\end{array}$ \\
\hline \multicolumn{2}{|c|}{ Environmental Influences on Hurricane Intensification } & 6. \\
\hline \multicolumn{2}{|l|}{$\begin{array}{l}\text { 7. Author(s) } \\
\text { Robert T. Merri11 }\end{array}$} & 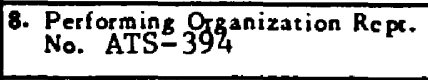 \\
\hline \multirow{2}{*}{\multicolumn{2}{|c|}{$\begin{array}{l}\text { 9. Petorming Organization Name and Address } \\
\text { Atmospheric Sclence Department } \\
\text { Colorado State University } \\
\text { Fort Collins, Colorado } 80523\end{array}$}} & 10. Project/Task/Work Unit No. \\
\hline & & $\begin{array}{l}\text { 11. Contract/Grant No. } \\
\text { ATM } 8419116\end{array}$ \\
\hline \multirow{2}{*}{\multicolumn{2}{|c|}{$\begin{array}{l}\text { 12. Sponsoting Organization Name and Address } \\
\text { NSF/NOAA Grant No. ATM-8419116 } \\
\text { National Science Foundation } \\
18 \text { th and G Streets, N.W., Washington, D.C. } 20550\end{array}$}} & $\begin{array}{l}\text { 13. Type of Report \& Period } \\
\text { Covered } \\
\text { Project Report }\end{array}$ \\
\hline & & 14. \\
\hline
\end{tabular}

15. Suppiementary Notes

\section{Abstracts}

Though qualitatively similar in structure, different hurricanes can attain differen peak intensities during their lifetimes. Forecasters and empiricists relate the intensity to the sea surface temperature and the "effectiveness" of the upper tropospheric outflow, but of fer no clear explanation of how the latter operates. Numerical modelers usually ignore the surrounding flow and emphasize interaction between the convective and vortex scales exclusively. This paper examines more closely the observed uppertropospheric environmental flow differences between hurricanes which intensify and those which fall to do so, and combines them with previously published empirical and modeling results into a general conceptual model of environmental influences on hurricane intenification.

17. Key lords and Document Analysis. 17a. Descriptors

Hurricane Intensification

Hurricanes

Hurricane Structure

Hurricane Dynamics

17b. Identiliers/Open-Eaded Terms

17c. cosATI Fie Id/Group

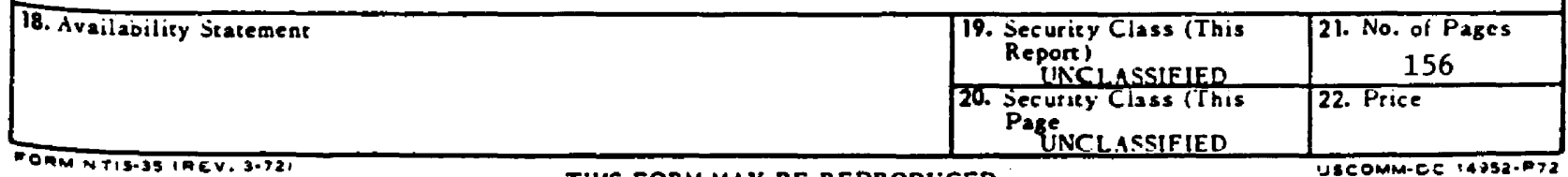


Author: Robert $T$. Herrill

(P.I.: Willex M. Gray)

ENVIRGARENTAL INFLUENCES ON RURRICANE INTENSIFICATION

Colorado State University

Department of Atmospheric Sclence

Fort Coll1ns, Colorado 80523

Subject Headings:

Hurricane Intensification Hurricanes

Hurricane Structure

Hurricane Dynanics

\section{NSF/NOMA Grant No. ATM 8419116}

Though qualitatively imllar in structure, different hurricanes can attaln different peak intensities during their ilfetimes. Forecasters and empiricists relate the Intensity to the sea surface temperature and the "effectiveness" of the upper tropoupheric outflow, but offer no elear explanation of hou the latcer operates. Numert al modelers usually lear explanation the surtoud vortex scales exclusively. Thls paper examlnes more closely the observed upper-tropospheric environmental flow differences between hurricanes which intensify and those which fall to do so, and combines them with previously published emplrical and modeling results into a general conceptual model of environmental influences on hurricane intensification.

Author: Robert T. Merrill

$$
\text { (P.I.: William M. Gray) }
$$

ENVI RONHENTAL INFLUENCES ON HURRICANE INTENSIFICATION

Colorado State Untversity

Department of Atmospheric Science

Fort Collins, colorado 80523

Subject Headings:

Hurricane Intensification

Hurricanes

Hurricane Structure

Hurrlcane Dynamics

\section{NSF/NOMA Grent No. ATM 8419116}

Though qualitatively simflar in structure, different hurricanes can attain different peak intensities during their 11 fetimes. Forecasters and empiricists relate the intensity to the sea surface temperature and

the "effectlveness" of the upper tropospheric outflow, but of fer no clear explanation of how the latter operates. Numerical modelers usually ignore the surrounding flow and emphasize interaction between the convective and vortex scales exclusively. Th1s paper examines more closely the observed upper-tropospheric environmental flow differences between hurricanes wh1ch Intensify and those which fall to do so, and combines them with previously published emplrical and modeling results into a general conceptual model of environmental influences on hurricane intensification.

Author: Robert T. Merrill

(P.I.: Willian M. Gray)

ENVIRONENTAL INFLUENCES ON HURRICANE INTENSIFICATION

Colorado State University

Department of Atmospheric Science

Fort Collins, Colorado 80523

Subject Headings:

Hurricane Intensification Hurricanes

Hurricane Structure

Hurricane Dynamic*

NSF/NOM Grant No. ATM 8419116

Though qualitatively similar in structure, different hurricanes can attain different peak intensitles during their 11 fetimes. Forecasters and empiricists relate the intensity to the sea surface temperature and the "effectlveness" of the upper troposperle outflow, but offer no clear the "effrect explanation of how the latcer operaces. Numerical modelers usually 1gnore und vortex scales exclusively. This paper examines more closely the observed upper-tropospher1c environmental flow differences between hurricanes wh1ch intensify and those which fall to do so, and combines them with previously published emplrical and modeling resules into a general conceptual model of environmental influences on hurricane intensification.

Author: Robert T. Merrl11

$$
\text { (P.I.: William M. Gray) }
$$

ENVIRONMENTAL INFLUENCES ON HURRICANE INTENSIFICATION

Colorado State Univeraity

Department of Atmospherlc Sc1ence

Fort Collins, Colorado 80523

NSF/NOM Grant No. ATM 8419116

Though qualitatively similar in sructure, different hurricanes can attain different peak intensities during their 11fetimes. Forecasters and empiricists relate the intens to the the "effectiveness" of the upper tropospheric outflow, but offer no clear explanation of how the latter operates. Numerical modelers usually ignore the surrounding flow and emphasize interaction between the convective and vortex scales exclusively. Th1s paper examines more closely the observed upper-tropospheric environmental flow differences between hurricanes which intensify and those which fall to do so, and combines them with previously published empirical and modeling results into general conceptual model of environmental influences on hurricane intensification.
Subject Headings: Hurr1cane Incenstefcation Hurricanes Hurricane Structure Hurricane Dynamica 